\title{
Epidemiology of neurodegenerative diseases in sub-Saharan Africa: a systematic review
}

\author{
Alain Lekoubou', Justin B Echouffo-Tcheuguii ${ }^{2,3}$ and Andre P Kengne $e^{4,5,6,7^{*}}$
}

\begin{abstract}
Background: Sub-Saharan African (SSA) countries are experiencing rapid transitions with increased life expectancy. As a result the burden of age-related conditions such as neurodegenerative diseases might be increasing. We conducted a systematic review of published studies on common neurodegenerative diseases, and HIV-related neurocognitive impairment in SSA, in order to identify research gaps and inform prevention and control solutions.

Methods: We searched MEDLINE via PubMed, 'Banque de Données de Santé Publique' and the database of the 'Institut d'Epidemiologie Neurologique et de Neurologie Tropicale' from inception to February 2013 for published original studies from SSA on neurodegenerative diseases and HIV-related neurocognitive impairment. Screening and data extraction were conducted by two investigators. Bibliographies and citations of eligible studies were investigated.

Results: In all 144 publications reporting on dementia ( $n=49$ publications, mainly Alzheimer disease), Parkinsonism $(P D, n=20)$, HIV-related neurocognitive impairment $(n=47)$, Huntington disease $(H D, n=19)$, amyotrophic lateral sclerosis (ALS, $n=15)$, cerebellar degeneration $(n=4)$ and Lewy body dementia $(n=1)$. Of these studies, largely based on prevalent cases from retrospective data on urban populations, half originated from Nigeria and South Africa. The prevalence of dementia (Alzheimer disease) varied between $<1 \%$ and $10.1 \%(0.7 \%$ and $5.6 \%)$ in population-based studies and from $<1 \%$ to $47.8 \%$ in hospital-based studies. Incidence of dementia (Alzheimer disease) ranged from 8.7 to 21.8/1000/year ( 9.5 to 11.1), and major risk factors were advanced age and female sex. HIV-related neurocognitive impairment's prevalence (all from hospital-based studies) ranged from $<1 \%$ to $80 \%$. Population-based prevalence of PD and ALS varied from 10 to 235/100,000, and from 5 to 15/100,000 respectively while that for Huntington disease was 3.5/100,000. Equivalent figures for hospital based studies were the following: PD (0.41 to $7.2 \%)$, ALS (0.2 to 8.0/1000), and $\operatorname{HD}(0.2 / 100,000$ to $46.0 / 100,000)$.

Conclusions: The body of literature on neurodegenerative disorders in SSA is large with regard to dementia and HIV-related neurocognitive disorders but limited for other neurodegenerative disorders. Shortcomings include few population-based studies, heterogeneous diagnostic criteria and uneven representation of countries on the continent. There are important knowledge gaps that need urgent action, in order to prepare the sub-continent for the anticipated local surge in neurodegenerative diseases.
\end{abstract}

Keywords: Neurodegenerative diseases, Parkinsonism, Dementia, HIV-related cognitive impairment, Sub-Saharan Africa

\section{Background}

Worldwide, populations are increasingly living longer including in developing countries, where the largest number of elderly people is currently found. In sub-Saharan Africa (SSA) (Figure 1), life expectancy at birth has increased by about 20 years between 1950 and 2010 [1]. During this same period, while the proportion of people

\footnotetext{
* Correspondence: andre.kengne@mrc.ac.za

${ }^{4}$ Department of Medicine, University of Cape Town, Cape Town, South Africa

${ }^{5}$ The George Institute for Global Health, Sydney, Australia

Full list of author information is available at the end of the article
}

aged 60 years and above has remained constant at around $5 \%$, the absolute number in this group has increased by about four folds from 9.4 million in 1950 (total population 179.5 million) to 40.3 million in 2010 (total population 831.5 million). In general, population ageing has been described as a more recent phenomenon in SSA, causing figures for this region to be well below the global average [1]. However, projections suggest that the gap in life expectancy between SSA and the world average, which was around 20 years in 2010, will drop to 10 years by 2050 . By this time, about $7.6 \%$ of the 
SSA population (estimated total 2.074 billion) will be aged 60 years and above, which in absolute number will translate into four times the 2010 estimates, and correspond approximately to 156.7 million people [2].

Population ageing is considered a global public health success, but also brings about new health challenges in the form of chronic diseases including cardiovascular diseases, cancers, as well as neurodegenerative disorders. A characterization and updated picture of the latter conditions in SSA is particularly important in view of a) the ongoing demographic transition and the resulting surge in the prevalence of neurodegenerative diseases in SSA; b) the successful roll-out of antiretroviral therapies in the region and the potential, yet unknown impact of long-term survival with HIV infection and related treatments on the occurrence of neurodegenerative disorders [3]; and c) lastly, the need for reliable data for health service planning. Recently, there have been efforts to summarize existing data for conditions like Parkinson disease (PD) $[4,5]$ dementia [6,7] or amyotrophic lateral sclerosis [8], but not for other common neurodegenerative disorders, while there are suggestions of possible African distinctiveness in their occurrence and features [9].

We systematically reviewed the published literature on common neurodegenerative disorders and HIV-related neurocognitive impairment among sub-Saharan Africans,

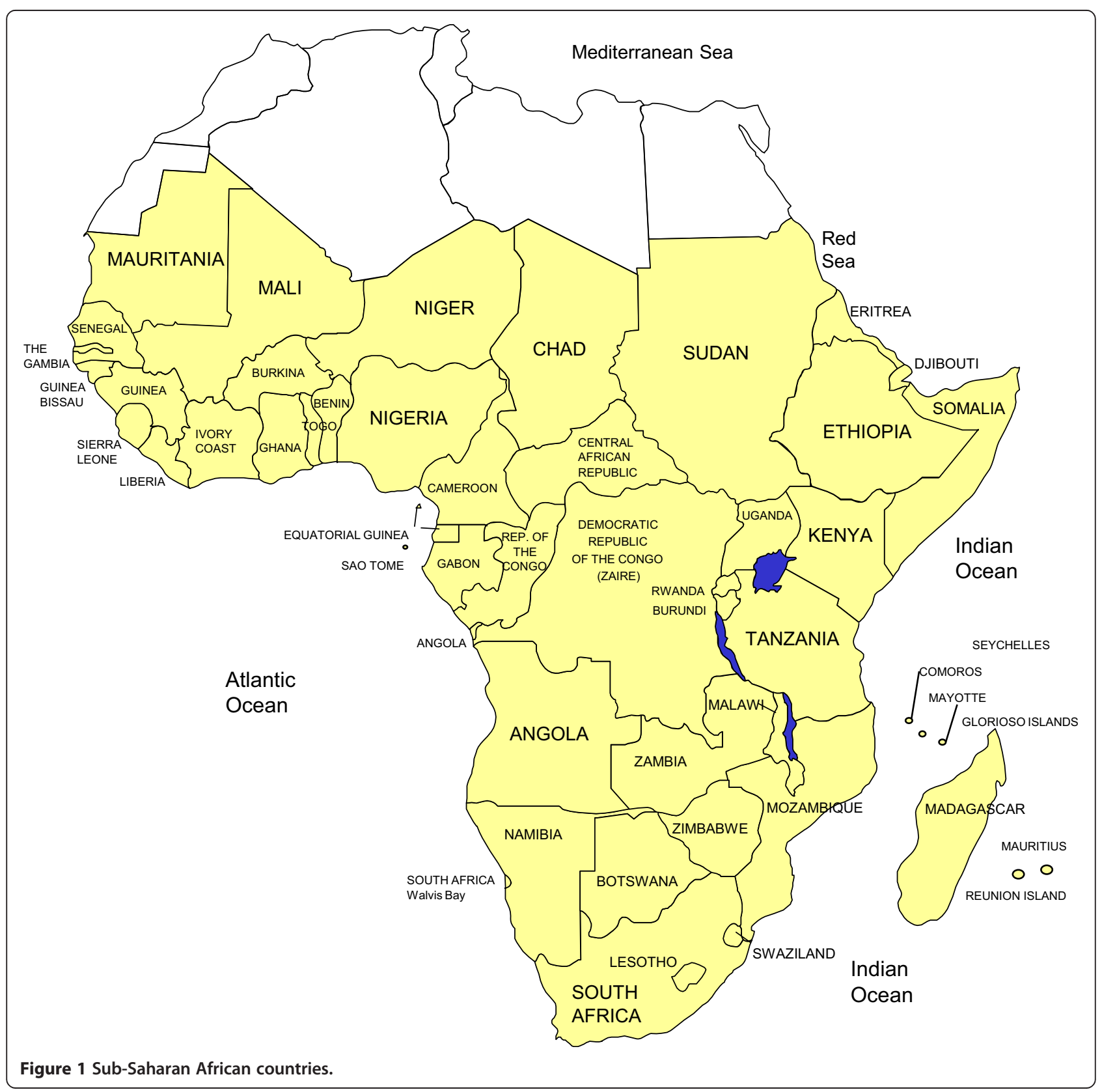


with the objective of describing their main features as well as clinical and public health implications.

\section{Methods}

\section{Data sources}

We searched MEDLINE via PubMed, and the French database 'Banque des Données en Santé Publique' (BDSP www.bdsp.ehesp.fr) for articles published until February 2013. In addition we searched the database of the 'Institut d'Epidemiologie Neurologique et de Neurologie Tropicale' (IENNT). We used a combination of relevant terms to search (in English for PubMed and in French for BDSP and IENNT), which are presented in Additional file 1 (except for IENNT searches for which we used 'neuroepidemiologie' and other themes referring to neurodegenerative diseases). Two evaluators (AL and JBE) independently identified articles and sequentially (titles, abstracts, and then full texts) screened them for inclusion (Figure 2). For articles without abstracts or without enough information in the abstract to make a decision, the full text, and where necessary supplemental materials, were reviewed before a decision was made. We supplemented the electronic searches by scanning the references lists of relevant publications, and identifying their citations through the ISI Web of Science, and by hand-searching all issues of the African Journal of Neurological Sciences. Disagreements were solved by consensus or review by a third investigator (APK).

\section{Study selection}

We included studies conducted in a country of the SSA region (Figure 1) that reported on the following neurodegenerative diseases among adults: Alzheimer's disease, fronto-temporal dementia, Lewy body dementia, vascular dementia, cortico-basal degeneration, multi system atrophy, Parkinson's disease (PD), amyotrophic lateral sclerosis (ALS), Huntington disease, cerebellar degeneration, and HIV-related neurocognitive impairment. We made no restriction by study design. We excluded duplicate publications, review articles, studies conducted exclusively in

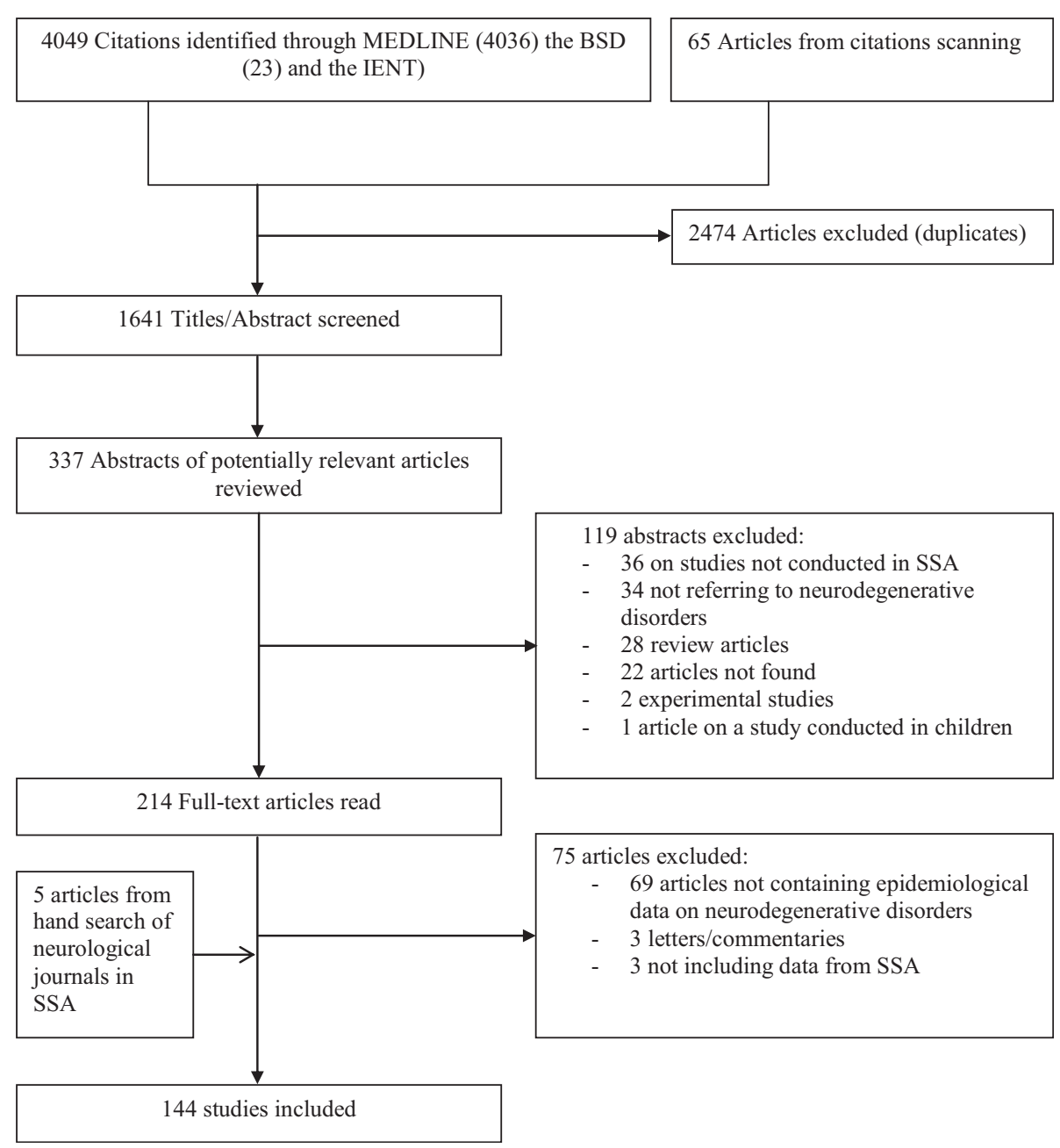

Figure 2 Flow of selection of studies for inclusion. 
pediatric populations, studies conducted exclusively on migrant populations of African descent living out of the continent. Figure 2 shows the study selection process.

We provide a rigorous appraisal of the overall data and the epidemiological studies in particular, and make recommendations regarding future approaches to measurement, notwithstanding the challenges involved in such undertakings.

\section{Data extraction, assessment, and synthesis}

Two reviewers (AL and JBE) independently conducted the data extraction from included studies. We extracted data on study settings, design, population characteristics, measures of disease occurrence (incidence and/or prevalence), and risk factors for the various conditions examined. Given the diversity of neurodegenerative pathologies and the heterogeneity of populations assessed, we did not use a particular framework for the assessment of the quality of studies. However, whenever population-based studies and hospital-based studies had been conducted for a condition, we relied more on the conclusions of population-based studies to address relevant questions, and appropriately reported the results. We conducted a narrative synthesis of the evidence.

\section{Results}

The study selection process is shown in Figure 2. A total of 4049 citations were identified through MEDLINE, the IENNT database and BDSP searches; 337 abstracts were evaluated in detail and 214 full-text publications reviewed. The final selection included 144 publications reporting on Parkinsonism (20 studies), dementia (49 publications), HIV-related neurocognitive impairment (47 publications), Huntington disease (19 studies), amyotrophic lateral sclerosis (15 studies), cerebellar degeneration (4 studies) and Lewy body dementia (1 study). These studies were published between 1955 and 2012, with about $50 \%$ conducted in only two countries: Nigeria and South Africa.

\section{Parkinson disease, other Lewy body diseases and fronto-temporal dementia}

Twenty studies reported on Parkinsonism (Table 1), including five community-based and sixteen hospitalbased. Four were case-control in design and all the others were cross-sectional studies, including reviews of medical records. These studies were conducted in seven countries including Nigeria (ten studies), South Africa (four studies), Tanzania (two studies), Ethiopia, Ghana, Cameroon and Zimbabwe (one studies each). The number of participants with PD ranged from two to 32 and the prevalence from ten to $235 / 100,000$ in community-based studies. The number of participants with Parkinsonism ranged from four to 397, and the prevalence of
Parkinsonism varied from 0.41 to $7.2 \%$ of neurological admissions/consultations in hospital-based studies. The proportion of men among those with PD ranged from 53 to $100 \%$, and age ranged from 30 to $>100$ years. Age at the clinical onset of the disease ranged from 17 to 90 years. The clinical types of the disease were largely dominated by Parkinson disease (38 to $100 \%$ ).

The most commonly used tool to diagnose PD was the UKPDS Brain bank criteria and population-based (hospital-based) prevalence for the studies that applied those criteria ranged from 40 to $235 / 100,000$ (11 to 69.4/1,000 neurological consultations). In general risk factors were not investigated across studies, although one study found that $38 \%$ of patients with Parkinsonism had atherosclerosis and $8 \%$ had encephalitis [18].

We found three cases of Lewy body dementia in a retrospective study in Nigeria, and one case in a retrospective study in Senegal representing respectively 1.2/ 100,000 of admission over a period of 10 years [30] and $7.5 / 1000$ of participants in a specialized memory clinic [31].

The prevalence of fronto-temporal dementia has been reported in two hospital-based studies conducted in Neuropsychiatric clinics in Nigeria (prevalence rate: 1.7/ 100,000 of all admissions) and in Senegal (prevalence rate: 7.5/1000 of all participants evaluated for memory impairment) [30,31].

\section{Dementia}

(Table 2) summarizes the 49 publications that reported on dementia. These include 18 hospital-based, 30 community-based publications and one publication from a nursing home. Two were case-control in design, seven were cohort-studies and 40 were cross-sectional, including two autopsy studies. These publications reported on studies conducted in eleven countries: Nigeria (33 publications), Senegal (four publications), Kenya and Tanzania (three publications each), Benin, Central African Republic, Congo republic, (two publications each), South Africa, Cameroon and Zambia (one publication each). In addition, there were seven publications on multicenter studies including African American participants in the USA and participants from African countries [32-37]. The overall study size varied from 56 to 2494 in community-based studies and from 23 to 240,294 in hospital-based investigations. The prevalence of dementia ranged from $<1 \%$ to $10.1 \%$ in population-based studies [32,34-57] and from $<1 \%$ to $47.8 \%$ in hospitalbased studies [16,21,30,33,38,58-69].

The proportion of men among those with dementia was 7.1 to $69.1 \%$. The mean age of participants ranged from 70.1 to 83.8 years. When provided, age at clinical diagnosis of disease ranged from 80.7 to 83.8 years. Alzheimer disease was the most common form of the disease, representing 57.4 to $89.4 \%$ of all cases 
Table 1 Overview of studies on Parkinsonism and risk factors in sub-Saharan African countries

\begin{tabular}{|c|c|c|c|c|c|c|c|c|}
\hline $\begin{array}{l}\text { Author, year of } \\
\text { publication }\end{array}$ & Country & Setting & $\begin{array}{l}\text { Design/period } \\
\text { of study }\end{array}$ & Population characteristics & Diagnosis criteria & Prevalence & $\begin{array}{l}\text { Profile of } \\
\text { parkinsonism } \\
\text { patients }\end{array}$ & Comments \\
\hline \multirow{3}{*}{$\begin{array}{l}\text { Bower [10], } \\
2005\end{array}$} & \multirow[t]{3}{*}{ Ethiopia } & \multirow[t]{3}{*}{ Hospital } & \multirow{3}{*}{$\begin{array}{l}\text { Cross-sectional } \\
2003-2004\end{array}$} & \multirow{3}{*}{$\begin{array}{l}720 \text { patients; } 109(15 \cdot 1 \%) \\
\text { with movement disorders } \\
\text { including } 71 \text { men; age } 52 \text { y. } \\
(13-80)\end{array}$} & \multirow[t]{3}{*}{ Not provided } & \multirow{3}{*}{$\begin{array}{l}72 / 1,000 \text { of all } \\
\text { admissions } \\
\text { (PD: 64/1,000) }\end{array}$} & $\mathrm{N}: 52 ; \mathrm{PD}: 88 \%$ & \multirow{3}{*}{$\begin{array}{l}\text { Review of medical files/ } \\
\text { outpatient neurology clinic. }\end{array}$} \\
\hline & & & & & & & $\begin{array}{l}\text { Age (at onset): } \\
57 y(30-80)\end{array}$ & \\
\hline & & & & & & & Men: $75 \%$ & \\
\hline \multirow{3}{*}{$\begin{array}{l}\text { Akinyemi [11], } \\
2008\end{array}$} & \multirow[t]{3}{*}{ Nigeria } & \multirow[t]{3}{*}{ Hospital } & \multirow{3}{*}{$\begin{array}{l}\text { Case-control } \\
\text { 2005-2005 }\end{array}$} & \multirow{3}{*}{$\begin{array}{l}51 \text { patients (men 37) with } \\
\text { PD and } 50 \text { controls }\end{array}$} & \multirow{3}{*}{$\begin{array}{l}\text { UKPDS Brain Bank } \\
\text { criteria }\end{array}$} & \multirow[t]{3}{*}{ NA } & N:51; PD: 100\% & \multirow{3}{*}{$\begin{array}{l}22 \% \text { patients with PD had } \\
\text { cognitive dysfunction, with } \\
\text { age at PD onset as sole } \\
\text { predictor of cognitive } \\
\text { dysfunction. }\end{array}$} \\
\hline & & & & & & & $\begin{array}{l}\text { Age (at onset): } \\
70 y(41-80)\end{array}$ & \\
\hline & & & & & & & Men:72\% & \\
\hline \multirow[t]{4}{*}{$\begin{array}{l}\text { Cosnett [12], } \\
1988\end{array}$} & \multirow[t]{4}{*}{$\begin{array}{l}\text { South } \\
\text { Africa }\end{array}$} & \multirow[t]{4}{*}{ Hospital } & \multirow[t]{4}{*}{$\begin{array}{l}\text { Cross-sectional } \\
1979-1985\end{array}$} & \multirow[t]{4}{*}{2638 patients } & \multirow{4}{*}{$\begin{array}{l}\text { Clinical (Bradykinesia, } \\
\text { rigidity, resting tremor } \\
\text { and postural instability) }\end{array}$} & \multirow[t]{4}{*}{$5.3 / 1,000$} & $\mathrm{~N}: 14 ;$ PD: $100 \%$ & $\begin{array}{l}\text { Retrospective review of } \\
\text { medical files/outpatient clinic }\end{array}$ \\
\hline & & & & & & & Age: NA & Blacks: 1.5/1000 \\
\hline & & & & & & & Men: NA & Indians: 12.6/1000 \\
\hline & & & & & & & & Whites: 23.1/1000 \\
\hline \multirow{4}{*}{$\begin{array}{l}\text { Dotchin [13] } \\
2008\end{array}$} & \multirow[t]{4}{*}{ Tanzania } & \multirow[t]{4}{*}{ Community } & \multirow[t]{4}{*}{ Cross-sectional } & \multirow[t]{4}{*}{161,071 inhabitants } & \multirow{4}{*}{$\begin{array}{l}\text { UKPDS Brain Bank } \\
\text { criteria }\end{array}$} & Overall: 40/100,000 & N: 32; PD:100\% & \multirow{14}{*}{$\begin{array}{l}\text { Prevalence is adjusted to } \\
\text { UK population. Mean } \\
\text { duration } 5.1 \mathrm{y}\end{array}$} \\
\hline & & & & & & Men: 64/100,000 & & \\
\hline & & & & & & women: 20/100,000 & $\begin{array}{l}\text { Age (at onset): } \\
69 y(29-90)\end{array}$ & \\
\hline & & & & & & & Men: $72 \%$ & \\
\hline \multirow{7}{*}{$\begin{array}{l}\text { Schoenberg } \\
\text { [14], } 1988\end{array}$} & Nigeria & Community & Cross-sectional & Black population aged & Clinical & Age adjusted: & $\mathrm{N}: 2 ; \mathrm{PD}: 100 \%$ & \\
\hline & & & & $40+3412$ participants & & $6 / / 100,000$ & Age: NA & \\
\hline & & & & & & & Men: NA & \\
\hline & USA & Community & Cross-sectional & Black population aged & Clinical & Age adjusted: & $\mathrm{N}: 12 ; \mathrm{PD}: 100 \%$ & \\
\hline & & & & $\begin{array}{l}\text { participants and } 5404 \\
\text { park }\end{array}$ & & & Age: NA & \\
\hline & & & & white participants. & & Blacks: $341 / 100,000$ & Men: NA & \\
\hline & & & & & & Whites: 352/100,000 & & \\
\hline Winkler [15], & Tanzania & Hospital & Cross-sectional & $\mathrm{n}=8676$ patients admitted & UKPDS Brain Bank & 1/1,000 (all patients) & N: 8; PD:37\% & \\
\hline & & & 2003 & $\begin{array}{l}\text { (740 with neurological } \\
\text { diseases) }\end{array}$ & criteria & 11/1,000 (Patients & Age: $\geq 32$ y & \\
\hline & & & & & & $\begin{array}{l}\text { with neurological } \\
\text { diseases }\end{array}$ & Men: $100 \%$ & \\
\hline & & Community & Cross-sectional & 1569 people, age & UKPDS Brain Bank & $235 / 100,000$ & $\mathrm{~N}: 0$ & None of the 18 screened- \\
\hline & & & $2003-2005$ & & & & & $\begin{array}{l}\text { positive was confirmed as } \\
\text { having PD. Poisson } \\
\text { distribution used to } \\
\text { estimate the prevalence. }\end{array}$ \\
\hline
\end{tabular}


Table 1 Overview of studies on Parkinsonism and risk factors in sub-Saharan African countries (Continued)

\begin{tabular}{|c|c|c|c|c|c|c|c|c|}
\hline \multirow{2}{*}{$\begin{array}{l}\text { Kengne [16], } \\
2006\end{array}$} & & & \multirow[t]{2}{*}{ 1993-2001 } & \multirow{2}{*}{\multicolumn{2}{|c|}{$\begin{array}{l}4041 \text { patients in a } \\
\text { neurology clinic145 (3.9\%) } \\
\text { had neurodegenerative } \\
\text { diseases }\end{array}$}} & \multirow{2}{*}{$\begin{array}{l}488 / 1,000 \text { of all } \\
\text { neurodegenerative } \\
\text { diseases; } 10.1 / 1,000 \\
\text { of all neurologic } \\
\text { consultation }\end{array}$} & Age: $15-84$ y & \multirow{2}{*}{$\begin{array}{l}4 \text { selected neurodegenerative } \\
\text { brain disorders: dementia, PD, } \\
\text { ALS, chorea }\end{array}$} \\
\hline & & & & & & & Men: $73.2 \%$ & \\
\hline \multirow{2}{*}{$\begin{array}{l}\text { Lombard } \\
{[17], 1978}\end{array}$} & \multirow[t]{2}{*}{ Zimbabwe } & \multirow[t]{2}{*}{ Hospital } & \multirow[t]{2}{*}{ Cross-sectional } & \multirow{2}{*}{$\begin{array}{l}\text { Total patients admitted: } \\
83,453 \text { blacks, } 34,952 \text { whites }\end{array}$} & \multirow[t]{2}{*}{ Not provided } & Blacks: $0.21 / 1,000$ & N: 50 (17 blacks) & \multirow{2}{*}{$\begin{array}{l}\text { Retrospective review of } \\
\text { medical files }\end{array}$} \\
\hline & & & & & & Whites: $2.83 / 1,000$ & Age/men: NA & \\
\hline \multirow{3}{*}{$\begin{array}{l}\text { Osuntokun [18], } \\
1979\end{array}$} & \multirow[t]{3}{*}{ Nigeria } & \multirow[t]{3}{*}{ Hospital } & Cross-sectional & \multirow[t]{3}{*}{217 patients with parkinsonism } & \multirow[t]{3}{*}{ Not provided } & \multirow[t]{3}{*}{ NA } & N: 217; PD 38\% & \multirow{3}{*}{$\begin{array}{l}\text { All patients evaluated by } \\
\text { the authors }\end{array}$} \\
\hline & & & \multirow[t]{2}{*}{ 1966-1976 } & & & & $\begin{array}{l}\text { Age: median } \\
51-70 \mathrm{y}\end{array}$ & \\
\hline & & & & & & & Men:75\% & \\
\hline \multirow{2}{*}{$\begin{array}{l}\text { Osuntokun [19], } \\
1987\end{array}$} & \multirow[t]{2}{*}{ Nigeria } & \multirow[t]{2}{*}{ Community } & Cross-sectional & \multirow{2}{*}{$\begin{array}{l}\text { Total participants surveyed: } \\
18,954\end{array}$} & \multirow[t]{2}{*}{ Not provided } & \multirow[t]{2}{*}{$10 / 100,000$} & N. $2 ;$ PD $100 \%$ & \multirow{2}{*}{$\begin{array}{l}\text { Screening Questionnaire } \\
\text { developed by author }\end{array}$} \\
\hline & & & 1985 & & & & Age/men: NA & \\
\hline \multirow{3}{*}{$\begin{array}{l}\text { Haylett [20], } \\
2012\end{array}$} & \multirow{3}{*}{$\begin{array}{l}\text { South } \\
\text { Africa }\end{array}$} & \multirow[t]{3}{*}{ Hospital } & \multirow[t]{3}{*}{ Cross-sectional } & \multirow{3}{*}{$\begin{array}{l}229 \text { patients with PD including } \\
163 \text { whites }(71 \%), 45 \text { mixed } \\
\text { ancestry }(20 \%), 17 \text { blacks } \\
(7 \%) \text { and } 4 \text { Indians ( } 2 \%)\end{array}$} & UKPDS Brain Bank & NA & N: 229; PD 100\% & Mutation in the Parkin gene \\
\hline & & & & & & & $\begin{array}{l}\text { Age (at onset): } \\
54 \text { y }(17-80)\end{array}$ & $\begin{array}{l}\text { Homozygous or compound } \\
\text { heterozygous mutations: } \\
7 \text { patients }\end{array}$ \\
\hline & & & & & & & Men: \% NA & Heterozygous variant: 7 \\
\hline Ekenze [21], & Nigeria & Hospital & Cross-sectional & 8440 admission in the & Not specified & $21.9 / 1000$ of al & $N: 14$ & \\
\hline & & & 2003-2007 & $\begin{array}{l}\text { medical ward; } 1249 \text { had } \\
\text { neurological diseases }\end{array}$ & & $\begin{array}{l}\text { neurological } \\
\text { admissions }\end{array}$ & Age $\geq 70$ y $(71 \%)$ & \\
\hline & & & & (men 640) & & & Men: $28.6 \%$ & \\
\hline Owolabi [22], & Nigeria & Hospital & Cross-sectional & 6282 admission in the & Clinical: any 3 out of & $4.1 / 1,000$ of all & $\mathrm{N}: 4$ & \\
\hline & & & 2005-2007 & $\begin{array}{l}\text { medical ward; } 980 \text { had } \\
\text { neurological diseases }\end{array}$ & $\begin{array}{l}\text { tremor, rigidity, Akinesia/ } \\
\text { bradikinesia/postural }\end{array}$ & $\begin{array}{l}\text { neurological } \\
\text { admissions }\end{array}$ & Age: (50-68) & \\
\hline & & & & (men 586) & and instability & & Men; 100\% & \\
\hline Okubadejo [23], & Nigeria & Hospital & Case-control & 33 participants (men 25, & Any 3 out of tremor, & NA & N: 33 & Case fatality rate was higher \\
\hline & & & & $\begin{array}{l}\text { mean age } 60 \text { y) with PD } \\
\text { and } 33 \text { match controls }\end{array}$ & $\begin{array}{l}\text { rigidity, Akinesia/ } \\
\text { bradikinesia/postural } \\
\text { and instability }\end{array}$ & & $\begin{array}{l}\text { Age (at onset): } \\
36-80 y\end{array}$ & $\begin{array}{l}\text { in PD ( } 25 \% \text { vs. } 7.1 \%) \text {, Factors } \\
\text { associated with increased } \\
\text { mortality: advanced age and }\end{array}$ \\
\hline & & & & & & & Men: $75 \%$ & disease severity \\
\hline Okubadejo [24], & Nigeria & Hospital & Case-control & 28 participants (men 21, & Any 2 out of tremor, & NA & N: 28; PD 100\% & Autonomic dysfunction rate \\
\hline & & & & $\begin{array}{l}\text { mean age } 63 \text { y) with PD } \\
\text { and } 28 \text { match controls }\end{array}$ & $\begin{array}{l}\text { rigidity, Akinesia/ } \\
\text { bradikinesia/postural } \\
\text { and instability exclusion }\end{array}$ & & $\begin{array}{l}\text { Age (at onset): } \\
37-76 \text { y }\end{array}$ & was higher in PD (61\% vs. 6\%), \\
\hline & & & & & $\begin{array}{l}\text { of other causes } \\
\text { of parkinsonism }\end{array}$ & & Men: $76 \%$ & \\
\hline Okubadejo [25], & Nigeria & Hospital & Cross-sectional & 124 participants with & Any 3 of the following: & $15 / 1,000$ of all & N: 98; PD 79\% & Other causes of parkinsonism \\
\hline & & & $1996-2006$ & $\begin{array}{l}\text { Parkinsonism in a } \\
\text { neurology clinic }\end{array}$ & $\begin{array}{l}\text { tremors, riglaity, } \\
\text { bradykinesia, and } \\
\text { postural or gait } \\
\text { abnormality }\end{array}$ & $\begin{array}{l}\text { neurological } \\
\text { consultations }\end{array}$ & $\begin{array}{l}\text { Age (at onset): } \\
61 y \text { Men: } 76.5 \%\end{array}$ & $\begin{array}{l}\text { n(\%): vascular/arug } \\
\text { induced/MSA/LBD: } 9(35) / 5 \\
(19) / 4(15) / 3(11)\end{array}$ \\
\hline
\end{tabular}


Table 1 Overview of studies on Parkinsonism and risk factors in sub-Saharan African countries (Continued)

\begin{tabular}{|c|c|c|c|c|c|c|c|c|}
\hline \multirow{3}{*}{$\begin{array}{l}\text { Keyser [26], } \\
2010\end{array}$} & \multirow{3}{*}{$\begin{array}{l}\text { South } \\
\text { Africa }\end{array}$} & \multirow[t]{3}{*}{ Hospital } & \multirow[t]{3}{*}{ Cross-sectional } & \multirow{3}{*}{$\begin{array}{l}154 \text { patients with PD } \\
\text { including } 51 \text { whites ( } 35 \%) \text {, } \\
45 \text { Afrikaners (31\%), } 29 \text { mixed } \\
\text { ancestry (20\%), } 17 \text { blacks } \\
(12 \%) \text { and } 3 \text { Indians ( } 2 \%) \text {. }\end{array}$} & \multirow{3}{*}{$\begin{array}{l}\text { UK Parkinson's Disease } \\
\text { UKPDS Brain Bank criteria }\end{array}$} & \multirow[t]{3}{*}{ NA } & N: 154; PD 100\% & \multirow{3}{*}{$\begin{array}{l}16 \text { sequence variants of the } \\
\text { PINK1gene identified: } 1 \\
\text { homozygous mutation (Y258X) } \\
2 \text { heterozygous missense } \\
\text { variants (P305A and E476K), } \\
\text { and } 13 \text { polymorphisms }\end{array}$} \\
\hline & & & & & & & Age (at onset): 52 y & \\
\hline & & & & & & & Men: $62 \%$ & \\
\hline \multirow{2}{*}{$\begin{array}{l}\text { Van Der Merwe } \\
\text { [27], } 2012\end{array}$} & \multirow{2}{*}{$\begin{array}{l}\text { South } \\
\text { Africa }\end{array}$} & \multirow[t]{2}{*}{ Hospital } & Cross-sectional & \multirow{2}{*}{$\begin{array}{l}111 \text { patients with early onset } \\
\text { PD (men 71) and 286 with } \\
\text { late onset PD (men 62\%) } \\
\text { from a movement } \\
\text { disorder clinic }\end{array}$} & \multirow{2}{*}{$\begin{array}{l}\text { UKPDS Brain Bank } \\
\text { criteria }\end{array}$} & \multirow[t]{2}{*}{ NA } & N: 397; PD 100\% & \multirow{5}{*}{$\begin{array}{l}\text { A positive family history was } \\
\text { associated with a younger } \\
\text { age at onset. }\end{array}$} \\
\hline & & & 2007-2011 & & & & $\begin{array}{l}\text { Age (at onset): } \\
57 \text { y Men: } 248\end{array}$ & \\
\hline \multirow[t]{3}{*}{ Femi [28], 2012} & \multirow[t]{3}{*}{ Nigeria } & \multirow[t]{3}{*}{ Hospital } & Cross-sectional & \multirow{3}{*}{$\begin{array}{l}1153 \text { participants in } 2 \\
\text { Neurologic clinics; } 96 \\
\text { (men: } 74 \text { ) had } \\
\text { parkinsonism }\end{array}$} & \multirow{3}{*}{$\begin{array}{l}\text { presence of at least three } \\
\text { of the four cardinal features } \\
\text { of tremors, rigidity, } \\
\text { bradykinesia, and postural } \\
\text { or gait abnormality }\end{array}$} & \multirow{3}{*}{$\begin{array}{l}69.4 / 1,000 \text { of all } \\
\text { neurological } \\
\text { consultations }\end{array}$} & N: 96; PD (83.3\%) & \\
\hline & & & \multirow[t]{2}{*}{$2007-2011$} & & & & Age: 58 y & \\
\hline & & & & & & & Men: 63.5\% & \\
\hline \multirow[t]{3}{*}{ Cilia [29], 2012} & \multirow[t]{3}{*}{ Ghana } & \multirow[t]{3}{*}{ Hospital } & \multirow[t]{3}{*}{ Case-control } & \multirow{3}{*}{$\begin{array}{l}54 \text { participants with } \\
\text { PD and } 46 \text { healthy } \\
\text { participants }\end{array}$} & \multirow{3}{*}{$\begin{array}{l}\text { UKPDS Brain Bank } \\
\text { criteria }\end{array}$} & \multirow[t]{3}{*}{ NA } & N: 54; PD 100\% & \multirow{3}{*}{$\begin{array}{l}\text { Leucine-rich repeat kinase } 2 \\
\text { (LRRK2) gene found in no } \\
\text { participants }\end{array}$} \\
\hline & & & & & & & $\begin{array}{l}\text { Age (at onset): } \\
59 \text { y (30-83) }\end{array}$ & \\
\hline & & & & & & & Men: 61\% & \\
\hline
\end{tabular}


Table 2 Overview of studies on dementia and risk factors in sub-Saharan Africa

\begin{tabular}{|c|c|c|c|c|c|c|c|}
\hline $\begin{array}{l}\text { Author, year } \\
\text { of publication }\end{array}$ & Country/setting & $\begin{array}{l}\text { Design/period } \\
\text { of study }\end{array}$ & Population characteristics & Diagnostic criteria & Incidence & Prevalence (\%) & Risk factors \\
\hline \multirow{2}{*}{$\begin{array}{l}\text { Lambo [58], } \\
1966\end{array}$} & Nigeria & \multirow{2}{*}{$\begin{array}{l}\text { Retrospective/ } \\
\text { Cross-sectional, } \\
\text { 1954-1963 }\end{array}$} & 328 participants ( $26 \% \geq 60$ y.) & \multirow[t]{2}{*}{ Not provided } & \multirow[t]{2}{*}{ NA } & Senile dementia*: & \multirow[t]{2}{*}{ NA } \\
\hline & Hospital & & 75 cases of dementia ( 21 men) & & & $\begin{array}{l}\text { Overall: 26\%, Men: } \\
\text { 18.9\% Women: } 30.5 \%\end{array}$ & \\
\hline \multirow{2}{*}{$\begin{array}{l}\text { Ben-Arie [39], } \\
1983\end{array}$} & South Africa & \multirow{2}{*}{$\begin{array}{l}\text { Cross-sectional, } \\
1982\end{array}$} & \multirow[t]{2}{*}{139 participants aged $\geq 65 \mathrm{y}$. } & \multirow[t]{2}{*}{ MMSE/ICD-8 codes } & \multirow[t]{2}{*}{ NA } & \multirow{2}{*}{$\begin{array}{l}\text { Any (severe) dementia } \\
8.6 \%(3.6 \%)\end{array}$} & \multirow[t]{2}{*}{ NA } \\
\hline & Community & & & & & & \\
\hline \multirow{2}{*}{$\begin{array}{l}\text { Makanjuola [59], } \\
1985\end{array}$} & Nigeria & \multirow{2}{*}{$\begin{array}{l}\text { Cross-sectional } \\
1979-1982\end{array}$} & \multirow{2}{*}{$\begin{array}{l}51 \text { ( } 5.2 \% \text { of new consultations); } \\
\text { age } \geq 60 \mathrm{y} \text {. }\end{array}$} & \multirow[t]{2}{*}{ ICD-9 codes } & \multirow[t]{2}{*}{ NA } & \multirow[t]{2}{*}{ Dementia 11.2\% } & \multirow[t]{2}{*}{ NA } \\
\hline & Hospital & & & & & & \\
\hline \multirow{2}{*}{$\begin{array}{l}\text { Gureje [60], } \\
1989\end{array}$} & Nigeria & \multirow{2}{*}{$\begin{array}{l}\text { Cross-sectional, } \\
1984\end{array}$} & \multirow[t]{2}{*}{1914 patients; } & \multirow[t]{2}{*}{ ICD- 9 codes } & \multirow[t]{2}{*}{ NA } & \multirow[t]{2}{*}{ No case of dementia } & \multirow[t]{2}{*}{ NA } \\
\hline & Community & & & & & & \\
\hline \multirow{2}{*}{$\begin{array}{l}\text { Ogunniyi [40], } \\
1992\end{array}$} & Nigeria & \multirow[t]{2}{*}{ Cross-sectional } & \multirow{2}{*}{$\begin{array}{l}930 \text { participants; age } \geq 40 \text { y. } \\
\text { ( } 293 \text { aged } \geq 65 \text { y.); No case } \\
\text { of dementia }\end{array}$} & \multirow[t]{2}{*}{ DSM-III-R criteria } & \multirow[t]{2}{*}{ NA } & \multirow[t]{2}{*}{ No case of dementia } & \multirow[t]{2}{*}{ NA } \\
\hline & Community & & & & & & \\
\hline $\begin{array}{l}\text { Osuntokun [61], } \\
1994\end{array}$ & $\begin{array}{l}\text { Nigeria, hospital } \\
\text { Autopsy study }\end{array}$ & $\begin{array}{l}\text { Cross-sectional } \\
\text { 1986- } 1987\end{array}$ & $\begin{array}{l}111 \text { brains autopsied including } \\
85 \text { patients aged } \leq 60 \mathrm{y} \text {. }\end{array}$ & $\begin{array}{l}\text { Beta A4 amyloid on } \\
\text { brain tissues }\end{array}$ & NA & $\begin{array}{l}\text { Heavy/moderate/mild } \\
\text { plaque load: 0/6.3/18.9\% }\end{array}$ & NA \\
\hline Osuntokun [41], & Nigeria, & Cross-sectional & 56 subjects ( 17 with dementia & Dementia -CSID & NA & APOE $\varepsilon 4$ allele in & NA \\
\hline & community & & and 12 with AD); age $\geq 65$ y. & $\begin{array}{l}\text { AD - NINCDS-ADRDA } \\
\text { criteria }\end{array}$ & & $\begin{array}{l}\text { dementia/AD/controls } \\
\text { 17.6/16.7/20.5\%. }\end{array}$ & \\
\hline Osuntokun [38], & Nigeria, hospital & Cross-sectional & 198 brains were autopsied & senile plaque, & NA & No evidence of NFT or & NA \\
\hline & Autopsy study & 1986- 1987 & Including $45(23 \%) \geq 65$ year & $\begin{array}{l}\text { neurofibrillary tangle, } \\
\text { and amyloid vascular } \\
\text { degeneration }\end{array}$ & & senile plaque & \\
\hline $\begin{array}{l}\text { Hendrie [32], } \\
1995\end{array}$ & $\begin{array}{l}\text { Nigeria, } \\
\text { community }\end{array}$ & Cross-sectional & $\begin{array}{l}2494 \text { participants, age } \geq 65 \text { y., } \\
\text { Dementia }-28, A D-18, \text { VaD - } 8 \text {. }\end{array}$ & $\begin{array}{l}\text { Dementia: CSID/DSM-III- } \\
\text { R/ICD-10/AD: NINCDS- }\end{array}$ & NA & $\begin{array}{l}\text { Dementia - Overall/ } \\
65-74 / 75-84 / \geq 85 \mathrm{y}:\end{array}$ & \\
\hline & & 1992-1993 & & & & $2.3 / 0 \cdot 9 / 2.7 / 9.6$ & \\
\hline & & & & & & $A D-1.4 / 0.5 / 1.7 / 5.9 \%$ & \\
\hline & Indianapolis-USA, & Cross-sectional & 2212participants, aged $\geq 65 \mathrm{y}$. & Dementia: CSID/DSM-III- & NA & Dementia Overall/ & NA \\
\hline & nursing home & $1992-1993$ & (nursing home) & ADRDA criteria & & $82 / 2.6 / 114 / 324 \%$ & \\
\hline & & & & & & $A D-6.2 / 1.6 / 8.0 / 28.8 \%$ & \\
\hline $\begin{array}{l}\text { Ogeng'o [33], } \\
1996\end{array}$ & Tanzania, hospital & Cross-sectional & $\begin{array}{l}12 \text { Non-demented subjects } \\
\text { aged } 45-83 \text { y. }\end{array}$ & $\begin{array}{l}\text { senile plaque, } \\
\text { neurofibrillary tangle, }\end{array}$ & NA & Amyloid $\beta$ plaques:17\% & NA \\
\hline 1996 & & Autopsy study & & $\begin{array}{l}\text { and cerebral amyloid } \\
\text { angiopathy }\end{array}$ & & $\begin{array}{l}\text { Neurofibrillary Tangles: } \\
\text { 17\%; Cerebral Amyloid } \\
\text { angiopathy: } 17 \%\end{array}$ & \\
\hline
\end{tabular}


Table 2 Overview of studies on dementia and risk factors in sub-Saharan Africa (Continued)

\begin{tabular}{|c|c|c|c|c|c|c|c|}
\hline & Kenya, hospital & $\begin{array}{l}\text { Cross-sectional } \\
\text { Autopsy study }\end{array}$ & $\begin{array}{l}20 \text { Non-demented subjects } \\
\text { aged } 45-70 \mathrm{y} \text {. }\end{array}$ & $\begin{array}{l}\text { Senile plaque, } \\
\text { neurofibrillary tangle, } \\
\text { and cerebral amyloid } \\
\text { angiopathy }\end{array}$ & NA & $\begin{array}{l}\text { Amyloid } \beta \text { plaques: } 15 \% \text {; } \\
\text { Neurofibrillary Tangles: } \\
\text { 15\%; Cerebral Amyloid } \\
\text { angiopathy: } 15 \%\end{array}$ & NA \\
\hline & $\begin{array}{l}\text { USA-Cleveland, } \\
\text { Hospital }\end{array}$ & $\begin{array}{l}\text { Cross-sectional/ } \\
\text { Autopsy study }\end{array}$ & $\begin{array}{l}20 \text { Non-demented subjects } \\
\text { aged } 48-84 \text { y. }\end{array}$ & $\begin{array}{l}\text { Senile plaque, } \\
\text { neurofibrillary tangle, } \\
\text { and cerebral amyloid } \\
\text { angiopathy }\end{array}$ & NA & $\begin{array}{l}\text { Amyloid } \beta \text { plaques: } \\
\text { 20\%; Neurofibrillary: } \\
\text { 15\%; Cerebral Amyloid } \\
\text { angiopathy: } 20 \%\end{array}$ & NA \\
\hline \multirow{3}{*}{$\begin{array}{l}\text { Ogunniyi [42], } \\
1997\end{array}$} & \multirow{3}{*}{$\begin{array}{l}\text { Nigeria, } \\
\text { community }\end{array}$} & Cross-sectional & \multirow{3}{*}{$\begin{array}{l}2494 \text { participants aged }>65 \text { y } \\
\text { screened, } 28 \text { with dementia. }\end{array}$} & Screening: (CI-D) & \multirow[t]{3}{*}{ NA } & \multirow{3}{*}{$\begin{array}{l}\text { Any/ AD/ vascular } \\
\text { dementia - 1.1/0.7/0.3\% }\end{array}$} & \multirow[t]{3}{*}{ N A } \\
\hline & & \multirow[t]{2}{*}{ 1992-1994 } & & $\begin{array}{l}\text { Dementia: DSM-III-R } \\
\text { and ICD-10 codes }\end{array}$ & & & \\
\hline & & & & AD: NINCDS-ADRDA & & & \\
\hline \multirow[t]{2}{*}{ Sayi [62], 1997} & Tanzania, hospital & Cross-sectional & $\begin{array}{l}24 \text { demented and } 286 \\
\text { non-demented participants } \\
\text { aged } 50-89 \text { y. }\end{array}$ & Swahili modified MMSE & NA & $\begin{array}{l}\text { Prevalence of } \varepsilon 4 \text { allele of } \\
\text { APOE: Demented - } 25 \% \text {; } \\
\text { non demented - } 21 \%\end{array}$ & NA \\
\hline & Kenya, hospital & Cross-sectional & $\begin{array}{l}22 \text { demented and } 60 \\
\text { non-demented participants } \\
\text { aged } \geq 65 \mathrm{y} \text {. }\end{array}$ & Swahili modified MMSE & NA & $\begin{array}{l}\text { Prevalence of } \varepsilon 4 \text { allele of } \\
\text { APOE: Demented - } 42 \% \text {, } \\
\text { non-demented - } 27 \%\end{array}$ & NA \\
\hline \multirow{2}{*}{$\begin{array}{l}\text { Baiyewu [63], } \\
1997\end{array}$} & \multirow{2}{*}{$\begin{array}{l}\text { Nigeria, Nursing } \\
\text { home }\end{array}$} & Cross-sectional & \multirow{2}{*}{$\begin{array}{l}23 \text { participants (in a nursing } \\
\text { home) aged 66-102 y.i } \\
11 \text { women }\end{array}$} & \multirow[t]{2}{*}{ DSM-III-R/AGECAT } & \multirow[t]{2}{*}{ NA } & \multirow{2}{*}{$\begin{array}{l}\text { Any dementia (AD) - } \\
47 \cdot 8 \%(26 \cdot 1 \%)\end{array}$} & \multirow[t]{2}{*}{ NA } \\
\hline & & 1994 & & & & & \\
\hline \multirow[t]{4}{*}{ Hall [34],1998 } & \multirow[t]{2}{*}{$\begin{array}{l}\text { Nigeria, } \\
\text { community }\end{array}$} & \multirow[t]{2}{*}{ Case-control } & \multirow{2}{*}{$\begin{array}{l}2494 \text { participants; age } \geq 65 \text { y.; } \\
423 \text { clinically assessed } \\
\text { after screening, }\end{array}$} & Screening: CSID & \multirow[t]{2}{*}{ NA } & \multirow[t]{2}{*}{$\begin{array}{l}18 \text { cases of possible or } \\
\text { probable AD } 1.4 \%\end{array}$} & $\begin{array}{l}\text { age }(O R=1.15 \\
95 \%\end{array}$ \\
\hline & & & & $\begin{array}{l}\text { Dementia: DSM-III-R/ICD- } \\
\text { 10/AD: NINCDS-ADRDA }\end{array}$ & & & $\begin{array}{l}\mathrm{Cl}=1.12-1.18) \\
\text { and female gender } \\
(\mathrm{OR}=13.9 ; 95 \% \\
\mathrm{Cl}=3.85-50.82)\end{array}$ \\
\hline & \multirow{2}{*}{$\begin{array}{l}\text { USA-Indianapolis, } \\
\text { community }\end{array}$} & \multirow[t]{2}{*}{ Case-control } & 2212 participants; age $\geq 65$ y.i & Screening: CSID & \multirow[t]{2}{*}{ NA } & \multirow{2}{*}{$\begin{array}{l}\text { Possible/probable } \\
\text { AD } 6.2 \%\end{array}$} & \multirow{2}{*}{$\begin{array}{l}\text { age, family history } \\
\text { of dementia, education; } \\
\text { rural residence }\end{array}$} \\
\hline & & & $\begin{array}{l}351 \text { clinically assessed after } \\
\text { screening }{ }_{i ;} 49 \text { (men 17) } \\
\text { diagnosed with } A D\end{array}$ & $\begin{array}{l}\text { Dementia: DSM-III-R/ICD- } \\
\text { 10/AD: NINCDS-ADRDA }\end{array}$ & & & \\
\hline \multirow{2}{*}{$\begin{array}{l}\text { Uwakwe [70], } \\
2000\end{array}$} & \multirow[t]{2}{*}{ Nigeria, Hospital } & Cross-sectional & \multirow{2}{*}{$\begin{array}{l}119 \text { participants; age } \geq 65 \text { y; } \\
3 \text { had dementia }\end{array}$} & \multirow{2}{*}{$\begin{array}{l}\text { Geriatric Mental State } \\
\text { and/ICD-10 }\end{array}$} & \multirow[t]{2}{*}{ NA } & \multirow[t]{2}{*}{$2.8 \%$} & \multirow[t]{2}{*}{ NA } \\
\hline & & 1995-1996 & & & & & \\
\hline \multirow{4}{*}{$\begin{array}{l}\text { Ogunniyi } \\
{[43], 2000}\end{array}$} & \multirow{4}{*}{$\begin{array}{l}\text { Nigeria, } \\
\text { community }\end{array}$} & \multirow{4}{*}{$\begin{array}{l}\text { Cross-sectional } \\
\text { 1992-1994 }\end{array}$} & 2494 participants, age $\geq 65$ y.i & Screening: CSID & NA & Any dementia $2.3 \%$ & Age (OR: 1.15), female \\
\hline & & & including 18 with AD, 8 with & Dementia: DSM-III-R/ICD-10 & & & with others (OR: 0.06) \\
\hline & & & vascular dementia & AD: NINCDS-ADRDA & & AD: $1.4 \%$ & \\
\hline & & & & & & $\begin{array}{l}\text { E4 allele in AD (normal } \\
\text { subjects) } 34.2 \%(21.8 \%)\end{array}$ & \\
\hline
\end{tabular}


Table 2 Overview of studies on dementia and risk factors in sub-Saharan Africa (Continued)

\begin{tabular}{|c|c|c|c|c|c|c|c|}
\hline & \multirow{3}{*}{$\begin{array}{l}\text { Indianapolis-USA, } \\
\text { community }\end{array}$} & Cross-sectional & \multirow{3}{*}{$\begin{array}{l}2212 \text { participants, age } \geq 65 \text { year; } \\
65 \text { with dementia including } \\
49 \text { with } A D, 10 \text { with } \\
\text { vascular dementia }\end{array}$} & Screening: CSID & \multirow[t]{3}{*}{ NA } & \multirow{3}{*}{$\begin{array}{l}\text { Dementia (AD) overall/ } \\
65-74 / 75-84 / \geq 85 y-8.2 \\
(6.2) / 2.62(1.58) / 11.4 \\
(8.0) / 32 \cdot 4 \%(28.8 \%)\end{array}$} & \multirow{3}{*}{$\begin{array}{l}\text { Age, rural residence, } \\
\text { family history of } \\
\text { dementia, education }\end{array}$} \\
\hline & & \multirow[t]{2}{*}{ 1992-1994 } & & Dementia: DSM-III-R/ICD-10 & & & \\
\hline & & & & AD: NINCDS-ADRDA & & & \\
\hline \multirow{5}{*}{$\begin{array}{l}\text { Hendrie [35], } \\
2001\end{array}$} & \multirow{3}{*}{$\begin{array}{l}\text { Nigeria, } \\
\text { community }\end{array}$} & \multirow{3}{*}{$\begin{array}{l}\text { Prospective cohort } \\
\text { Baseline survey } \\
\text { in 1992-1993 }\end{array}$} & \multirow{3}{*}{$\begin{array}{l}2459 \text { participants included } \\
\text { after the first visit; } 1303 \\
\text { (men 461) completed the } \\
\text { follow-up; age } \geq 65 \mathrm{y} \text {. }\end{array}$} & Screening: CSID & \multirow{2}{*}{$\begin{array}{l}\text { Dementia: } \\
13.5 / 1,000\end{array}$} & \multirow[t]{3}{*}{ NA } & \multirow[t]{3}{*}{ NA } \\
\hline & & & & Dementia: DSM-III-R/ICD-10 & & & \\
\hline & & & & AD: NINCDS-ADRDA & AD: $11.5 / 1000$ & & \\
\hline & \multirow{2}{*}{$\begin{array}{l}\text { USA-Indianapolis, } \\
\text { community }\end{array}$} & Prospective cohort & \multirow{2}{*}{$\begin{array}{l}2147 \text { African-Americans } \\
\text { included after the first } \\
\text { visit; } 1321 \text { (men 417) } \\
\text { completed the follow-up; } \\
\text { age } \geq 65 \mathrm{y} \text {. }\end{array}$} & Screening: CSID & Dementia (AD) & \multirow[t]{2}{*}{ NA } & \multirow[t]{2}{*}{ NA } \\
\hline & & $\begin{array}{l}\text { Baseline survey } \\
\text { in 1992-1993 }\end{array}$ & & $\begin{array}{l}\text { Dementia: DSM-III-R/ICD- } \\
\text { 10/AD: NINCDS-ADRDA }\end{array}$ & $\begin{array}{l}32.4 / 1,000 \\
(25.2 / 1,000)\end{array}$ & & \\
\hline \multirow{2}{*}{$\begin{array}{l}\text { Baiyewu [44], } \\
2002\end{array}$} & \multirow{2}{*}{$\begin{array}{l}\text { Nigeria, } \\
\text { community }\end{array}$} & \multirow[b]{2}{*}{$\begin{array}{l}\text { Prospective cohort } \\
\text { baseline survey } \\
\text { in 1992-1993 }\end{array}$} & 2487 participants; age $\geq 65$ y:; & Screening: CSID & \multirow{2}{*}{$\begin{array}{l}\text { Conversion } \\
\text { from CIND } \\
\text { to dementia } \\
16 \cdot 1 \% \text {; From } \\
\text { CIND to normal } \\
25 \cdot 3 \%\end{array}$} & \multirow[t]{2}{*}{ NA } & \multirow[t]{2}{*}{ Sex } \\
\hline & & & $\begin{array}{l}423 \text { clinically assessed after } \\
\text { screening; } 152 \text { diagnosed } \\
\text { with CIND; } 28 \text { (men } 7 \text { ) with } \\
\text { dementia, } 87 \text { followed } \\
\text { up for } 2 \text { years. }\end{array}$ & Dementia: DSM-III-R/ICD-10 & & & \\
\hline \multirow{4}{*}{$\begin{array}{l}\text { Perkins [36], } \\
2002\end{array}$} & \multirow{2}{*}{$\begin{array}{l}\text { Ibadan-Nigeria } \\
\text { community }\end{array}$} & \multirow{2}{*}{$\begin{array}{l}\text { Prospective, } \\
1992-1993\end{array}$} & 2487 participants; age $\geq 65 \mathrm{y} ;$ & Screening: CSID & \multirow[t]{4}{*}{ NA } & \multirow[t]{2}{*}{$1.8 \%$} & \multirow{2}{*}{$\begin{array}{l}\text { Dementia associated } \\
\text { with mortality }\end{array}$} \\
\hline & & & $\begin{array}{l}\text { 423clinically assessed } \\
\text { after screening }\end{array}$ & Dementia: DSM-III-R/ICD-10 & & & \\
\hline & \multirow[t]{2}{*}{$\begin{array}{l}\text { Indianapolis-USA, } \\
\text { Community }\end{array}$} & \multirow{2}{*}{$\begin{array}{l}\text { Prospective } \\
\text { Baseline survey } \\
\text { in 1992-1993 }\end{array}$} & $\begin{array}{l}2212 \text { participants; } \\
\text { aged } \geq 65 \text { y.; }\end{array}$ & Screening: CSID & & \multirow[t]{2}{*}{$4.9 \%$} & \multirow{2}{*}{$\begin{array}{l}\text { Dementia associated } \\
\text { mortality (adjusted } \\
\text { RR: } 2 \cdot 05 \text { ) }\end{array}$} \\
\hline & & & $\begin{array}{l}342 \text { clinically assessed } \\
\text { after screening }\end{array}$ & Dementia: DSM-III-R/ICD-10 & & & \\
\hline \multirow[t]{4}{*}{ Lane [37], 2003} & $\begin{array}{l}\text { Nigeria } \\
\text { Community }\end{array}$ & $\begin{array}{l}\text { Prospective } 8.7 \text { y } \\
\text { follow up Baseline }\end{array}$ & $\begin{array}{l}968 \text { participants } \\
\text { ( } 271 \text { aged } \geq 75 \text { y.); }\end{array}$ & Screening: CSID & NA & NA & $\begin{array}{l}\text { ApoEk4 alleles not } \\
\text { associated with }\end{array}$ \\
\hline & & $1992-1993$ & $\begin{array}{l}\text { 23with dementia } \\
\text { at follow-up }\end{array}$ & Dementia: DSM-III-R/ICD-10 & & & Increased mortality \\
\hline & Indianapolis-USA, & Prospective $9.5 \mathrm{y}$. & 353 participants (17 4 aged & Screening: CSID & NA & NA & ApoEє4 associated \\
\hline & community & Baseline 1992-1993 & $\begin{array}{l}\text { ats y.j, 1/ with dementia } \\
\text { at follow-up }\end{array}$ & Dementia: DSM-III-R/ICD-10 & & & $\begin{array}{l}\text { With increased mortality } \\
\text { for patient under } 75 \text { year }\end{array}$ \\
\hline Ogunniyi [45], & Nigeria, & Cross-sectional/ & 98 demented subjects; & Screening: CSID & NA & AD: $82 \%$ of all cases & NA \\
\hline & & & & Dementia: DSM-III-R/ICD-10 & & VaD: $11.1 \%$ of all cases & \\
\hline Kengne [16], & Cameroon, & Cross sectional, & 4041 neurologic consultations & Not provided & NA & 0.4\% (all neurologic & NA \\
\hline & Hospital & 1993-2001 & $\begin{array}{l}145 \text { with neurodegenerative } \\
\text { diseases }\end{array}$ & & & $\begin{array}{l}\text { (neurodegenerative } \\
\text { diseases) }\end{array}$ & \\
\hline & & & $\begin{array}{l}16 \text { (men 14) with dementia, } \\
\text { mean age } 67.8 \mathrm{y} \text {. }\end{array}$ & & & & \\
\hline
\end{tabular}


Table 2 Overview of studies on dementia and risk factors in sub-Saharan Africa (Continued)

\begin{tabular}{|c|c|c|c|c|c|c|c|}
\hline \multirow{3}{*}{$\begin{array}{l}\text { Gureje [46], } \\
2006\end{array}$} & \multirow{3}{*}{$\begin{array}{l}\text { Nigeria, } \\
\text { Community }\end{array}$} & \multirow{3}{*}{$\begin{array}{l}\text { Cross-sectional, } \\
\text { 2003-2004 }\end{array}$} & \multirow{3}{*}{$\begin{array}{l}2152 \text { participants at baseline } \\
\text { with a respondent rate of } \\
74 \% \text { ( } 1904 \text { participants). } \\
\text { Aged } 65 \text { year or older. }\end{array}$} & \multirow{3}{*}{$\begin{array}{l}\text { adapted 10-Word Delay } \\
\text { Recall Test (10-WDRT)10 }\end{array}$} & \multirow[t]{3}{*}{ NA } & Overall: 10.1\%; & \multirow{3}{*}{$\begin{array}{l}\text { Female gender, } \\
\text { Increasing age, alcohol }\end{array}$} \\
\hline & & & & & & Female: $14.6 \%$ & \\
\hline & & & & & & Men: $7.0 \%$ & \\
\hline \multirow{2}{*}{$\begin{array}{l}\text { Gureje [71], } \\
2006\end{array}$} & \multirow{2}{*}{$\begin{array}{l}\text { Nigeria } \\
\text { Community }\end{array}$} & \multirow[t]{2}{*}{ Cross-sectional, } & \multirow{2}{*}{$\begin{array}{l}2245 \text { DNA samples, } \\
830 \text { had a diagnosis }\end{array}$} & Screening: CSID & \multirow[t]{2}{*}{ NA } & Any dementia (16.9\% & \multirow{2}{*}{$\begin{array}{l}\text { E4 allele in AD } \\
\text { (normal subjects) } \\
26 \cdot 0 \%(21 \cdot 7 \%)\end{array}$} \\
\hline & & & & Dementia: DSM-III-R/ICD-10 & & AD: $14.8 \%$ & \\
\hline \multirow{5}{*}{$\begin{array}{l}\text { Ogunniyi [72], } \\
2006\end{array}$} & \multirow{2}{*}{$\begin{array}{l}\text { Nigeria, } \\
\text { Community }\end{array}$} & \multirow[t]{2}{*}{ Case-control } & \multirow{2}{*}{$\begin{array}{l}62 \text { participants with AD } \\
\text { (Men } 16.1 \% \text {, mean age } 82 \text { y) } \\
\text { and } 461 \text { non demented } \\
\text { (men } 33.2 \% \text {, mean age } 77 \text { y) }\end{array}$} & Screening: CSID & \multirow[t]{2}{*}{ NA } & & Age (OR 1 · 07) \\
\hline & & & & $\begin{array}{l}\text { Dementia: DSM-III-R/ICD- } \\
\text { 10/AD: NINCDS-ADRDA }\end{array}$ & & & $\begin{array}{l}\text { Rural to age (OR } 2 \cdot 93) \\
\text { Hypertension (OR } 0 \cdot 33)\end{array}$ \\
\hline & \multirow{3}{*}{$\begin{array}{l}\text { Indianapolis-USA, } \\
\text { Community }\end{array}$} & \multirow[t]{3}{*}{ Case-control } & \multirow{3}{*}{$\begin{array}{l}89 \text { participants with } A D \\
\text { (men } 30.3 \%, \text { mean age } 83 \text { y), } \\
\text { mean age } 77 \text { y) and } 381 \text { non } \\
\text { demented (Men } 31.2 \%, \\
\text { mean age } 78 \text { y) }\end{array}$} & Screening: CSID & \multirow[t]{3}{*}{ NA } & & Age (OR 1.09 \\
\hline & & & & & & & Rural to age (OR 2.08) \\
\hline & & & & $\begin{array}{l}\text { Dementia: DSM-III-R/ICD- } \\
\text { 10/AD: NINCDS-ADRDA }\end{array}$ & & & \multirow[t]{4}{*}{$\begin{array}{l}\text { Alcohol consumption } \\
\text { (OR 0.49) }\end{array}$} \\
\hline \multirow{3}{*}{$\begin{array}{l}\text { Uwakwe [64], } \\
2006\end{array}$} & \multirow{3}{*}{$\begin{array}{l}\text { Nigeria, } \\
\text { Community }\end{array}$} & Cross-sectional & \multirow{3}{*}{$\begin{array}{l}30 \text { patients (men 12) with } \\
\text { dementia and their } \\
\text { caregivers (total } 30 \text { ) }\end{array}$} & \multirow[t]{3}{*}{ Not provided } & \multirow[t]{3}{*}{ NA } & N:52; & \\
\hline & & 2003-2005 & & & & AD: not provided & \\
\hline & & & & & & Men: 12 & \\
\hline \multirow{3}{*}{$\begin{array}{l}\text { Ochayi [47], } \\
2006\end{array}$} & \multirow{3}{*}{$\begin{array}{l}\text { Nigeria, } \\
\text { Community }\end{array}$} & \multirow{3}{*}{$\begin{array}{l}\text { Cross-sectional } \\
2002\end{array}$} & \multirow[t]{3}{*}{280 participants; age $\geq 65$ y:; } & \multirow[t]{3}{*}{ CSID } & NA & Overall dementia: 6.4\% & Female gender, \\
\hline & & & & & & $65-74$ year old: $5.2 \%$ & Lower body mass \\
\hline & & & & & & $\geq 85$ year $16 \%$ & \\
\hline Hall [48], 2006 & $\begin{array}{l}\text { Nigeria, } \\
\text { Community }\end{array}$ & Cross-sectional & $\begin{array}{l}1075 \text { participants; age } \geq 70 \text { y. } \\
29 \text { (men 5) with } A D \text {, }\end{array}$ & NINCDS-ADRDA & NA & NA & $\begin{array}{l}\text { Total- or LDL- } \\
\text { cholesterol in } \\
\text { individuals without } \\
\text { the APOE-ع4 allele }\end{array}$ \\
\hline $\begin{array}{l}\text { Uwakwe [73], } \\
2009\end{array}$ & $\begin{array}{l}\text { Nigeria, } \\
\text { community }\end{array}$ & Cross-sectional & $\begin{array}{l}914 \text { (men } 432 \text { ) participants, } \\
\text { age } \geq 65 \text { y; } 87 \text { with } \geq 2 \text { tests } \\
\text { memory tests impaired }\end{array}$ & $\begin{array}{l}\text { Memory impairment } \\
\text { assessed by MMS, CISD } \\
\text { and } 10 \text { word list immediate } \\
\text { and delayed recall }\end{array}$ & NA & $9.9 \%$ & NA \\
\hline Guerchet [50], & Benin Community & Cross-sectional & 502 (men 156) participants, & Screening: CSI-D & NA & Cognitive impairment & Age, current depressive \\
\hline & & & $\begin{array}{l}\text { aged } \geq 65 \text { y; } 52 \text { with } \\
\text { cognitive impairment }\end{array}$ & Dementia: DSM-IV & & $\begin{array}{l}\text { Overall: } 10.4 \% \text {; men } \\
7.7 \text { women } 11.5 \%\end{array}$ & $\begin{array}{l}\text { disorder, absence } \\
\text { of the APOE } \varepsilon 2\end{array}$ \\
\hline & & & 13 (men 1) with dementia & AD: NINCDS-ADRDA & & $\begin{array}{l}\text { Dementia Overall: } \\
2.5 \% \text {, men } 0.6 \% \\
\text { women } 3.4 \%\end{array}$ & \\
\hline Toure [67], 2009 & Senegal & Cross-sectional & 872 participants; age $\geq 55$ y. & DSM-IV-R & NA & Overall 6.6\% & Age, social isolation, \\
\hline & Hospital & $2004-2005$ & 58 cases of dementia & & & & $\begin{array}{l}\text { epilepsy, family } \\
\text { history of dementia, } \\
\text { Parkinson's disease }\end{array}$ \\
\hline & Burkina Fasso & Cross-sectional & & DSM-IV & NA & & NA \\
\hline
\end{tabular}


Table 2 Overview of studies on dementia and risk factors in sub-Saharan Africa (Continued)

\begin{tabular}{|c|c|c|c|c|c|c|c|}
\hline $\begin{array}{l}\text { Napon [68], } \\
2009\end{array}$ & Hospital & & $\begin{array}{l}15815 \text { (2396) out (in) participants; } \\
\text { age } \geq 15 \text { y.; } 72 \text { (and } 53 \text { inpatients) } \\
\text { with dementia; AD: } 7 ; \text { VaD: } 19 \text { cases }\end{array}$ & & & $\begin{array}{l}\text { outpatients: } 0.45 \% \\
\text { inpatients: } 0.22 \%\end{array}$ & \\
\hline \multirow{5}{*}{$\begin{array}{l}\text { Guerchet [49], } \\
2010\end{array}$} & \multirow{3}{*}{$\begin{array}{l}\text { Central African } \\
\text { Republic } \\
\text { Community }\end{array}$} & Cross-sectional & \multirow{2}{*}{$\begin{array}{l}509 \text { interviewed; } 496 \text { (men 218) } \\
\text { included in final sample, age } \geq 65 \mathrm{y} \text {. }\end{array}$} & Screening: CSID & \multirow[t]{5}{*}{ NA } & \multirow{3}{*}{$\begin{array}{l}\text { Overall: } 8.1 \% \text {, men } 2.7 \% \text {, } \\
\text { women } 12.2 \%\end{array}$} & \multirow[t]{3}{*}{ NA } \\
\hline & & 2008-2009 & & Dementia: DSM-IV & & & \\
\hline & & & $\begin{array}{l}188 \text { with cognitive impairment and } \\
40 \text { (men 6) with dementia (mean } \\
\text { age } 76 \text { y.); } 33 \text { (men 3) with } A D \\
\text { and } 7 \text { (men 2) with VaD }\end{array}$ & $\begin{array}{l}\text { AD: NINCDS-ADRDA } \\
\text { Hachinski scale, }\end{array}$ & & & \\
\hline & \multirow{2}{*}{$\begin{array}{l}\text { Republic of } \\
\text { Congo } \\
\text { Community }\end{array}$} & Cross-sectional & \multirow{2}{*}{$\begin{array}{l}546 \text { interviewed; } 520 \text { (men 198) } \\
\text { included in final sample, } \\
\text { age } \geq 65 \text { y. } 148 \text { with cognitive } \\
\text { impairment and } 35 \text { (men 9) } \\
\text { with dementia (mean age } 79 \text { y.); } \\
24 \text { (men 7) with AD and } 11 \\
\text { (men 3) with VaD }\end{array}$} & \multirow{2}{*}{$\begin{array}{l}\text { CSID/ DSM-IV and } \\
\text { NINCDS-ADRDA } \\
\text { Hachinski scale }\end{array}$} & & \multirow{2}{*}{$\begin{array}{l}\text { Overall: } 6.7 \% \text {, men } 4.5 \% \text {, } \\
\text { women } 8.1 \%\end{array}$} & \multirow[t]{2}{*}{ NA } \\
\hline & & $2008-2009$ & & & & & \\
\hline \multirow[t]{2}{*}{ Chen [65], 2010} & Kenya & \multirow[t]{2}{*}{ Cross-sectional } & 100 participants; age $\geq 65 \mathrm{y}$. & \multirow{2}{*}{$\begin{array}{l}\text { CSI-D using a version } \\
\text { in Kikuyu. }\end{array}$} & \multirow[t]{2}{*}{ NA } & Apo $\varepsilon 4$ allele frequency: & \multirow[t]{2}{*}{ NA } \\
\hline & Hospital & & $\begin{array}{l}84 \text { controls (men 38) and } 16 \\
\text { with dementia participants } \\
\text { (men 7) }\end{array}$ & & & $\begin{array}{l}\text { Demented } 31.3 \% \\
\text { non-demented } 32.2 \%\end{array}$ & \\
\hline \multirow{2}{*}{$\begin{array}{l}\text { Ekenze [21], } \\
2010\end{array}$} & Nigeria & Cross-sectional & \multirow{2}{*}{$\begin{array}{l}8440 \text { admissions; } 1249 \text { (men 640) } \\
\text { with neurological diseases } \\
\text { (age range18-83 y.); } 38 \text { (men 23) } \\
\text { with dementia }\end{array}$} & \multirow[t]{2}{*}{ Not specified } & \multirow[t]{2}{*}{ NA } & \multirow[t]{2}{*}{$3 \%$} & \multirow[t]{2}{*}{ NA } \\
\hline & Hospital & 2003-2007 & & & & & \\
\hline \multirow{2}{*}{$\begin{array}{l}\text { Siddiqi [69], } \\
2009\end{array}$} & Zambia & Cross-sectional & \multirow{2}{*}{$\begin{array}{l}443 \text { inpatients (men 219); } \\
\text { median age } 39 \text { y., } 67 \text { with } \\
\text { HIV; } 368 \text { outpatients (men 168); } \\
\text { median age } 39 \text { y., } 58 \text { with HIV; } \\
36 \text { with dementia }\end{array}$} & \multirow[t]{2}{*}{ Not specified } & \multirow[t]{2}{*}{ NA } & Dementia: & \multirow{2}{*}{$\begin{array}{l}\text { Dementia in HIV }+ \\
\text { patients } 8(13.8 \%) \text { vs. } \\
\text { general population } \\
9(2.9 \%)(p=0.002)\end{array}$} \\
\hline & Hospital & 2006 & & & & Overall: $4.4 \%$ & \\
\hline \multirow[t]{5}{*}{ Yusuf [74], 2011} & \multirow{5}{*}{$\begin{array}{l}\text { Nigeria } \\
\text { Community }\end{array}$} & \multirow[t]{5}{*}{ Cross-sectional } & \multirow{3}{*}{$\begin{array}{l}322 \text { participants (men 128); } \\
\text { mean age: } 75.5 \text { y }\end{array}$} & \multirow{2}{*}{$\begin{array}{l}\text { Screening: } \\
\text { CSID/CERAD/SDT }\end{array}$} & \multirow[t]{5}{*}{ NA } & Dementia: $2.8 \%$ & \multirow[t]{5}{*}{ Age } \\
\hline & & & & & & AD: $1.9 \%$ & \\
\hline & & & & $\begin{array}{l}\text { Dementia: DSM-IV } \\
\text { and ICD-10 }\end{array}$ & & VaD: $0.6 \%$ & \\
\hline & & & \multirow[t]{2}{*}{$\begin{array}{l}9 \text { cases of dementia (men 3); } \\
\text { mean age: } 82.4 \text { y }\end{array}$} & $\begin{array}{l}\text { LBD: McKhan clinical } \\
\text { criteria }\end{array}$ & & & \\
\hline & & & & $\begin{array}{l}\text { FTD: McKeith clinical } \\
\text { criteria }\end{array}$ & & & \\
\hline \multirow{2}{*}{$\begin{array}{l}\text { Gureje [51], } \\
2011\end{array}$} & Nigeria, & Prospective Cohort & 2,149 participants at baseline & 10-Word Delayed Recall & $21.80 / 1,000$ & NA & Poor social engagement, \\
\hline & & & $\begin{array}{l}1,408 \text { at } 39 \text { months follow-up; } 85 \\
\text { (among } \geq 65 \mathrm{y} \text {.) developed dementia }\end{array}$ & Test (cut off of 18) & & & $\begin{array}{l}\text { rural residence, low } \\
\text { economic status, female } \\
\text { gender, age. }\end{array}$ \\
\hline
\end{tabular}


Table 2 Overview of studies on dementia and risk factors in sub-Saharan Africa (Continued)

\begin{tabular}{|c|c|c|c|c|c|c|c|}
\hline \multirow[t]{2}{*}{$\begin{array}{l}\text { Ogunniyi [52], } \\
2011\end{array}$} & \multirow[t]{2}{*}{$\begin{array}{l}\text { Nigeria } \\
\text { Community }\end{array}$} & Cohort study & \multirow{2}{*}{$\begin{array}{l}1559 \text { participants aged }>65 \text { year } \\
\text { without dementia a baseline. } 136 \\
\text { (men 33) with dementia (mean } \\
\text { age } 83.1 \text { y.) at follow-up; } 255 \\
\text { with } \mathrm{MCl}\end{array}$} & \multirow[t]{2}{*}{$\begin{array}{l}\text { Dementia: DSM-III-R } \\
\text { and ICD-10 }\end{array}$} & $\begin{array}{l}\text { Dementia: } \\
\text { 8.72/1,000/year }\end{array}$ & \multirow[t]{2}{*}{ NA } & \multirow[t]{2}{*}{ Low BMI } \\
\hline & & 1992-2007 & & & $\begin{array}{l}\text { MCl: 16.35/ } \\
\text { 1000/year }\end{array}$ & & \\
\hline \multirow{3}{*}{$\begin{array}{l}\text { Ogunniy [53], } \\
2011\end{array}$} & \multirow{3}{*}{$\begin{array}{l}\text { Nigeria } \\
\text { Community }\end{array}$} & \multirow{3}{*}{$\begin{array}{l}\text { prospective cohort } \\
\text { baseline } 1992\end{array}$} & 2718 participants interviewed & \multirow{3}{*}{$\begin{array}{l}\text { Dementia: DSM-III-R } \\
\text { and ICD-10 }\end{array}$} & \multirow{3}{*}{$\begin{array}{l}\text { Dementia/AD/NaD } \\
\text { (per } 1,000 / \text { year) } \\
11.50 / 9.50 / 1.10\end{array}$} & \multirow{3}{*}{ NA } & \multirow[t]{3}{*}{ Higher SBP, DBP and PP } \\
\hline & & & 1753 (age $\geq 65 y$.) in the final sample & & & & \\
\hline & & & $\begin{array}{l}120 \text { (men } 30 \text { ) with dementia } \\
\text { (mean age } 83.8 \text { y.); } 99 \text { with } A D ; \\
11 \text { with VaD }\end{array}$ & & & & \\
\hline Paraïso [56], & \multirow[t]{4}{*}{ Benin Community } & Cross-sectional & \multirow{2}{*}{$\begin{array}{l}\text { 1,139 (men 523) participants; } \\
\text { age } \geq 65 \text { y:; } 42 \text { (men 13) with } \\
\text { dementia (mean age } 79 \cdot 1 \text { y) }\end{array}$} & Screening: CSI-D & \multirow[t]{4}{*}{ NA } & \multirow{2}{*}{$\begin{array}{l}\text { Dementia Overall } 3.7 \% \\
\text { men } 1.1 \% \text { women: } 2.5 \%\end{array}$} & \multirow[t]{4}{*}{ NA } \\
\hline \multirow[t]{3}{*}{2011} & & 2008 & & Dementia: DSM-IV & & & \\
\hline & & & \multirow[t]{2}{*}{32 with AD, 105 with CIND } & AD: NINCDS-ADRDA & & AD Overall 2.8\% & \\
\hline & & & & VaD: NINCDS-AIREN & & VD Overall $0.8 \%$ & \\
\hline Amoo [30], & \multirow[t]{2}{*}{ Nigeria } & \multirow[t]{2}{*}{ Cross-sectional } & \multirow[t]{2}{*}{ 240,294 participants } & \multirow[t]{2}{*}{ Dementia: ICD-10 } & \multirow[t]{6}{*}{ NA } & Dementia: 45/100,000 & \multirow[t]{6}{*}{ NA } \\
\hline 2011 & & & & & & $A D ; 25 \cdot 8 / 100,000$ & \\
\hline & \multirow[t]{4}{*}{ Hospital } & \multirow[t]{4}{*}{ 1998-2007 } & \multirow{3}{*}{$\begin{array}{l}108 \text { (men } 51 \text { ) with dementia } \\
\text { (mean age: } 70.1 \text { ); } 62 \text { (men 24) } \\
\text { with } A D ; 18 \text { (men 13) with VaD; } \\
4 \text { (men 2) with mixed forms; }\end{array}$} & ADNINCDS - ADRDA & & \multirow[t]{4}{*}{ VaD: $7 \cdot 4 / 100,000$} & \\
\hline & & & & VaD: NINCDS -AIRENS & & & \\
\hline & & & & LBD: McKeith criteria, & & & \\
\hline & & & $\begin{array}{l}4 \text { (men 2) with FTD; } 3 \text { (men 0) } \\
\text { with DLB; } 13 \text { (men 2) with } \\
\text { unclassified dementia }\end{array}$ & $\begin{array}{l}\text { FTD: Lund and } \\
\text { Manchester Criteria }\end{array}$ & & & \\
\hline \multirow{4}{*}{$\begin{array}{l}\text { Ndiaye [31], } \\
2011\end{array}$} & Senegal & Cross-sectional & 132 patients seen at a memory & Screening: "Test du & NA & MCl: $14.4 \%$ & NA \\
\hline & & $2004-2005$ & 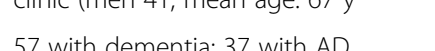 & HodKinson test & & & \\
\hline & & & 10 with $\mathrm{VaD}, 5$ with FTD and & MCl: Petersen criteria & & AD: $64.7 \%$ of all cases & \\
\hline & & & & Dementia: DSM-IV & & of dementia & \\
\hline $\begin{array}{l}\text { Coume [75], } \\
2012\end{array}$ & Senegal & Cross-sectional & $\begin{array}{l}872 \text { (men 546) participants } \\
\text { aged }>55 \mathrm{y} \text {; mean age } 67 \cdot 2 \mathrm{y}\end{array}$ & Test du Senegal & NA & $\begin{array}{l}\text { Cognitive } \\
\text { impairment } 10.8 \%\end{array}$ & NA \\
\hline & Hospital & $2004-2005$ & $\begin{array}{l}94 \text { (men 65) with cognitive } \\
\text { impairment ( } 74 \text { aged }>=65 \text { y) }\end{array}$ & & & & \\
\hline Baiyewu [54]., & Nigeria & Cross-sectional/ & 21 (men 4) participants with & Screening: CSID & NA & NA & NA \\
\hline & & & age 82.8 y.) & $\begin{array}{l}\text { Dementia: } \\
\text { DSM-III-R/ICD-10 }\end{array}$ & & & \\
\hline & Community & & $\begin{array}{l}53 \text { (men 4) with cognitive } \\
\text { impairment (mean age } 80.9 \text { ); } \\
34 \text { (men 6) with dementia } \\
\text { (mean age } 83.3 \text { y) }\end{array}$ & AD: NINCDS-ADRDA & & & \\
\hline
\end{tabular}


Table 2 Overview of studies on dementia and risk factors in sub-Saharan Africa (Continued)

\begin{tabular}{|c|c|c|c|c|c|c|c|}
\hline \multirow[t]{2}{*}{ Toure [66], 2012} & Senegal & Cross-sectional & 507 participants; age $\geq 65 \mathrm{y}$. & $\begin{array}{l}\text { Screening: Aging in } \\
\text { Senegal Questionnaire }\end{array}$ & NA & $8.9 \%$ & \multirow{2}{*}{$\begin{array}{l}\text { advanced age } \\
\text { (Age } \geq 80 \text { y, OR } 4.3, \\
95 \% \text { CI 1.4-13), illiteracy, } \\
\text { epilepsy, family } \\
\text { history of dementia }\end{array}$} \\
\hline & Hospital & $2004-2005$ & 45 with dementia & DSM-IV-R & & & \\
\hline \multirow{2}{*}{$\begin{array}{l}\text { Longdon [57], } \\
2012\end{array}$} & Tanzania & Cross-sectional & \multirow{2}{*}{$\begin{array}{l}1198 \text { (men 525) participants; } \\
\text { age } \geq 70 \text { y; } 78 \text { with dementia }\end{array}$} & Screening: CSI-D & \multirow[t]{2}{*}{ NA } & \multirow[t]{2}{*}{$6.4 \%$} & \multirow[t]{4}{*}{ Advanced age } \\
\hline & Community & 2010 & & DSM-IV-R & & & \\
\hline \multirow{2}{*}{$\begin{array}{l}\text { Onwuekwe [76], } \\
2012\end{array}$} & Nigeria & Cross-sectional & $\begin{array}{l}135 \text { participants (men: } 79 \text { ), } \\
\text { aged between } 16-76 \text { y }\end{array}$ & \multirow{2}{*}{$\begin{array}{l}\text { MMSE (cut off of } \\
17 \text { for MCl) }\end{array}$} & \multirow[t]{2}{*}{ NA } & \multirow[t]{2}{*}{ MCl: $5.9 \%$} & \\
\hline & Hospital & 2004 & 8 with $\mathrm{MCl}$ & & & & \\
\hline \multirow{4}{*}{$\begin{array}{l}\text { Guerchet [55], } \\
2012\end{array}$} & $\begin{array}{l}\text { Central African } \\
\text { Republic, Congo }\end{array}$ & Cross-sectional & \multirow{2}{*}{$\begin{array}{l}509 \text { interviewed; } 496 \text { (men 218) } \\
\text { included in final sample; age } \\
\geq 65 \text { y.; } 188 \text { with cognitive } \\
\text { impairment }\end{array}$} & \multirow{4}{*}{$\begin{array}{l}\text { Dementia: DSM-IV-R/AD: } \\
\text { NINCDS-ADRDA }\end{array}$} & \multirow[t]{4}{*}{ NA } & Dementia: $7.4 \%$ & \multirow{4}{*}{$\begin{array}{l}\text { Hypertension, low BMI, } \\
\text { depressive symptoms, } \\
\text { change of residence, } \\
\text { age }(\mathrm{OR} 2.59,95 \% \mathrm{Cl} \text {, } \\
\text { early death of one } \\
\text { parent, female gender }\end{array}$} \\
\hline & \multirow[t]{3}{*}{ Community } & 2008-2009 & & & & \multirow[t]{3}{*}{ AD: $5.6 \%$} & \\
\hline & & & $\begin{array}{l}546 \text { interviewed; } 520 \text { (men 198) } \\
\text { included in final sample; age } \\
\geq 65 \text { y:; } 148 \text { with cognitive } \\
\text { impairment }\end{array}$ & & & & \\
\hline & & & $\begin{array}{l}\text { Overall } 75 \text { (men } 15 \text { ) had dementia } \\
18 \text { with vascular dementia }\end{array}$ & & & & \\
\hline
\end{tabular}

AD: Alzheimer's disease; APOE: Apolipoprotein E; ICD: International Classification of Disease; BMI: Body Mass Index; Cl: confidence Interval; CIND: Cognitive Impairment and No Dementia; CSID: Community Screening Interview for Dementia; DSM-III-R: Diagnostic and Statistical Manual $3^{\text {rd }}$ edition revised; MMSE: Mini Mental State Examination; NA: Not available; NFT: Neurofibrillary tangle; NINCDS/ADRDA: National Institute of Neurological and Communicative Disorders and Stroke and the Alzheimer's Disease and Related Disorders Association; OR: Odd ratio; SCEB: short cognitive evaluation battery; USA: United States of America; VaD: Vascular dementia; y: years. 
$[30-32,34,42,45,55,56,63,71,74]$, followed by vascular dementia 5.7 to $31.0 \%$ of cases $[30,31,45,56,74]$. Four publications in Nigeria provided incidence data for dementia ranging from 8.7 to 21.8 cases per 1000 per year [35,51-53]. Incidence of Alzheimer disease ranged from 9.5 to 11.5 per 1000 per year [35,53].

The most commonly used tool for dementia screening was the Community Screening Interview for Dementia (CSID) questionnaire applied in 20 publications [32,34,36, 37,41-43,45-47,49,50,54,56,65,70]. The diagnosis of dementia mainly relied on the DSM-III-R/DSM-IV and ICD-10 classification [30,32,34-37,40,42-46,52-54,63,70]. The diagnosis of Alzheimer's disease was based on the National Institute of Neurological and Communicative Disorders and Stroke and the Alzheimer's Disease and Related Disorders Association (NINCDS/ADRDA) criteria $[30,32,34,35,41,43,48,50,52-56,75]$. Population-based studies that used DSM-III/DSM-IV and ICD-10 for dementia reported prevalences ranging from 1.1 to $8.1 \%$ [32,35,42, 49,55-57,65,67,74] (ref 13, 16, 23, 30, 36-38, 48, 50, 118). Likewise the prevalence of Alzheimer's disease ranged from 0.7 to $5.6 \%$ based on NINCDS/ADRDA criteria $[35,42,55]$.

Risk factors for dementia were reported in 14 publications. The following were associated with an increased risk of dementia: age (twelve publications), female sex (five publications), low body mass index (three publications), anxiety/depression (three publications), hypertension (three publications), social isolation (two publications), lifetime history of alcohol consumption, elevated total- or LDL cholesterol in those without Apo E $\varepsilon 4$ (one publication), low socio-economic status, history of stroke and family history of dementia (one publication). The following characteristics were inversely associated with dementia: living with others, use of non-steroidal antiinflammatory drugs and absence of Apo E ع2. Some risk factors were more strongly related to the disease. These include age, which increased the risk of dementia by five to $16 \%$ across groups [34,43], but this effect was much higher after the age of 60 years, more than $100 \%$ increase risk especially after the age of $75[46,50,51,55,66,67]$. Female sex, low level of education ( $<6$ years), rural residence and family history increased the risk of dementia by $>100 \%[34,43,46,55,56,66]$.

\section{HIV-related neurocognitive impairment}

Fifty-one hospital-based studies (47 publications) reported on HIV-related neurocognitive impairment (Table 3), of which ten were case-control, six cohort and 31 crosssectional. These studies were conducted in 14 countries including South Africa (14 studies), Uganda (eight studies), Nigeria (six studies), Zambia and Kenya (four studies each), Cameroon and Democratic republic of Congo (three studies each) Ethiopia and Malawi (two studies each), Central African Republic, Botswana, Guinea Bissau, Tanzania and Zimbabwe (one study each). A total of 33 out of the 47 selected publications were published during the last 5 years and only 7 before 2000 . The absolute number of participants with HIV-related dementia ranged from 0 to 396, with a prevalence ranging from $0 \%$ to $80 \%$.

The diagnostic tools used to identify HIV-related dementia were variable, making comparison between studies less reliable. However, the International HIV Dementia Scale (IHDS) [89,95,97,105,107-110,112,113,120,121] and the Sloan Memorial Kettering scale $[86,89,90,98]$ were frequently used. Studies that used the IHDS reported a prevalence ranging from 21.1 to $80 \%$. The mean/median age of participants ranged from 31 to 40 years for those with HIV-related dementia, and men represented $25 \%$ to $56 \%$ of this group. In the nine studies that investigated etiological factors, the identified determinants of HIVrelated dementia were: low level of CD4 count (four studies), low level of education, and advanced age (three studies), comorbid psychiatric conditions (two studies each), advance clinical stage (two studies), male sex, HIVsubtype and duration of disease (one study each). The most commonly reported risk factors of HIV associated dementia were the level of CD4 count $[89,97,112,120,121]$ and the clinical stage of disease $[97,121]$.

\section{Amyotrophic lateral sclerosis and cerebellar degeneration} Fifteen studies (12 retrospective, 2 cross-sectional and 1 case-series) (Table 4) including 13 hospital and two community-based studies on amyotrophic lateral sclerosis (ALS) have been conducted in 9 SSA countries including Nigeria (four studies), Senegal (three studies), Ethiopia (2 studies), Zimbabwe, Kenya, South Africa, Sudan, Cameroon and Ivory coast (one study each). The number of participants with ALS ranged from two to 73 . Two community-based studies provided a prevalence of $15 / 100,000$ and 5/100,000 respectively in Nigeria [19] and in Ethiopia [122]. Five hospital-based studies provided prevalence figures: between 0.2 and 8.0/1000 of all neurologic consultation/admission [16,21,122-126]. The method of ascertainment of ALS was variable across studies, but electromyography was done in four of the fifteen studies included [125-129]. The proportion of men among those with ALS was 57.6 to $100 \%$. The age of those with ALS ranged from 12 to 84 years. When provided, the age at the clinical onset of ALS ranged from 12 to 71 years and the time to diagnosis from 3 months to more than 15 years. In general, risk factors for ALS were not investigated across studies.

One retrospective study in Nigeria reported on two cases (a 32 year old male and a 42 year old female) of cerebellar degeneration among $2 \cdot 1$ million admissions over a period of 25 year [14]. One study in Rwanda reported on a family of 33 members, with 15 (including 
Table 3 Overview of studies on HIV-related dementia and risk factors in sub-Saharan

\begin{tabular}{|c|c|c|c|c|c|c|c|}
\hline $\begin{array}{l}\text { Author, year } \\
\text { of publication }\end{array}$ & Country/setting & $\begin{array}{l}\text { Design/study } \\
\text { period }\end{array}$ & Population characteristics & Diagnostic criteria & Prevalence & Risk factors & Comments \\
\hline $\begin{array}{l}\text { Belec [77], } \\
1989\end{array}$ & $\begin{array}{l}\text { Central African } \\
\text { republic, } \\
\text { Hospital }\end{array}$ & $\begin{array}{l}\text { Cross-sectional } \\
1987\end{array}$ & $\begin{array}{l}93 \text { HIV + participants; age and } \\
\text { sex not specified }\end{array}$ & Not reported & HAND: 3 cases $(3.2 \%)$ & NA & $\begin{array}{l}\text { No neuro-imaging or } \\
\text { neuropathological } \\
\text { studies }\end{array}$ \\
\hline $\begin{array}{l}\text { Howlet [78], } \\
1989\end{array}$ & $\begin{array}{l}\text { Tanzania, } \\
\text { hospital }\end{array}$ & $\begin{array}{l}\text { Cross-sectional } \\
1985-1988\end{array}$ & $\begin{array}{l}200 \text { (men 129) HIV + } \\
\text { participants; mean age: } 32 \text { y }\end{array}$ & $\begin{array}{l}\text { Decline of memory and } \\
\text { other functions }\end{array}$ & $\begin{array}{l}\text { Dementia complex: } \\
54 \%\end{array}$ & NA & \\
\hline $\begin{array}{l}\text { Turnbull [79], } \\
1991\end{array}$ & South Africa & $\begin{array}{l}\text { Cross-sectional } \\
1982-1983\end{array}$ & $\begin{array}{l}27 \text { haemophilic patients } \\
\text { with HIV infection }\end{array}$ & $\begin{array}{l}\text { Battery of neuropsychological } \\
\text { tests: Rey complex figure, } \\
\text { Babcock story, digit span, } \\
\text { WAIS }\end{array}$ & $\begin{array}{l}\text { HAND: } 4 \text { cases } \\
(14.8 \%)\end{array}$ & NA & \\
\hline $\begin{array}{l}\text { Perriëns [80], } \\
1992\end{array}$ & $\begin{array}{l}\text { Democratic } \\
\text { republic of } \\
\text { Congo } \\
\text { Hospital }\end{array}$ & $\begin{array}{l}\text { Cross sectional } \\
2008\end{array}$ & $\begin{array}{l}104 \text { (men 48) HIV + } \\
\text { participants; mean age: } \\
34.3 \text { y.; } 92 \text { (men 53) HIV- } \\
\text { participants; mean age } 44 \text { y } \\
9 \text { (men 5) HIV + with HAND }\end{array}$ & $\begin{array}{l}\text { WHO operational criteria/ } \\
\text { American Academy of } \\
\text { neurology criteria }\end{array}$ & $\begin{array}{l}\text { HIV Associated } \\
\text { |Dementia Complex. } \\
8.7 \%\end{array}$ & NA & No neuro-imaging study \\
\hline \multirow[t]{2}{*}{ Maj [81], 1994} & $\begin{array}{l}\text { Kenya } \\
\text { Hospital }\end{array}$ & $\begin{array}{l}\text { Cross sectional } \\
\text { 1990-1991 }\end{array}$ & $\begin{array}{l}65 \text { (men 49) HIV- participants; } \\
\text { mean age: } 30 \text { y.; } 66 \text { (men 42) } \\
\text { asymptomatic HIV + participants; } \\
\text { mean age } 30.7 ; 72 \text { (men 48) } \\
\text { symptomatic HIV + participants; } \\
\text { mean age: } 33.2 \text { y }\end{array}$ & ICD-10/DSM-IV & $\begin{array}{l}\text { Dementia } \\
\text { HIV- } 0 \\
\text { Asymptomatic } \\
\text { HIV + } 0 \\
\text { Symptomatic } \\
\text { HIV + } 6(\%)\end{array}$ & NA & \\
\hline & $\begin{array}{l}\text { Democratic } \\
\text { republic of } \\
\text { Congo } \\
\text { Hospital }\end{array}$ & & $\begin{array}{l}85 \text { ( men 48) HIV- participants; } \\
\text { mean age: } 33.9 \text { y; } 52 \text { (men 33) } \\
\text { asymptomatic HIV + participants; } \\
\text { mean age } 32.3 \text { y.; } 68 \text { (men 35) } \\
\text { symptomatic HIV + participants; } \\
\text { mean age: } 33.8 \text { y }\end{array}$ & ICD-10/DSM-IV & $\begin{array}{l}\text { Dementia } \\
\text { HIV- } 0 \\
\text { Asymptomatic } \\
\text { HIV }+0 \\
\text { Symptomatic } \\
\text { HIV + (5.9\%) }\end{array}$ & NA & \\
\hline \multirow{2}{*}{$\begin{array}{l}\text { Carson [82], } \\
1998\end{array}$} & Kenya & Cross sectional & \multirow{2}{*}{$\begin{array}{l}78 \text { (men 52) HIV + participants; } \\
\text { mean age: } 29.9 \text { y.; } 138 \text { (men } 114 \text { ) } \\
\text { HIV- participants; mean age } 29.8 \text { y. }\end{array}$} & \multirow{2}{*}{$\begin{array}{l}\text { Revised WAIS, Trails A and } \\
\text { Trails B tests, Digit span, } \\
\text { Delayed word and d } \\
\text { recognition }\end{array}$} & \multirow[t]{2}{*}{ NA } & \multirow[t]{2}{*}{ NA } & \multirow{2}{*}{$\begin{array}{l}\text { No difference in } \\
\text { neuropsychiatric test } \\
\text { performance between } \\
\text { HIV + and HIV- }\end{array}$} \\
\hline & Hospital & 1994 & & & & & \\
\hline \multirow{3}{*}{$\begin{array}{l}\text { Sebit [83], } \\
1995\end{array}$} & Kenya & Cross sectional & \multirow{2}{*}{$\begin{array}{l}191 \text { participants, } 72 \text { (men 48) } \\
\text { symptomatic HIV + (mean } \\
\text { age } 33.2 \text { y.), } 66 \text { (men 42) } \\
\text { asymptomatic HIV + } \\
\text { (mean age } 30.7 \text { ) and } 65 \\
\text { (men 49) HIV- (mean age } 30 \text { y.) }\end{array}$} & \multirow{2}{*}{$\begin{array}{l}\text { WHO operational criteria/ } \\
\text { American Academy of } \\
\text { neurology criteria }\end{array}$} & Mental disorders: & \multirow[t]{2}{*}{ NA } & \multirow{2}{*}{$\begin{array}{l}\text { No specific data for HIV } \\
\text { associated neurocognitive } \\
\text { disorders }\end{array}$} \\
\hline & Hospital & 1990-1991 & & & $\begin{array}{l}\text { Symptomatic } \\
\text { HIV }+7.1 \% \text {, } \\
\text { Asymptomatic HIV + } \\
4.5 \%, \text { HIV }-0\end{array}$ & & \\
\hline & $\begin{array}{l}\text { Democratic } \\
\text { republic of } \\
\text { Congo (DRC)/ } \\
\text { Hospital }\end{array}$ & & $\begin{array}{l}190 \text { participants, } 68 \text { (men 35) } \\
\text { symptomatic HIV + (mean } \\
\text { age } 33.8 \text { y.), } 52 \text { (men 33) } \\
\text { asymptomatic HIV + (mean }\end{array}$ & $\begin{array}{l}\text { WHO operational criteria/ } \\
\text { American Academy of } \\
\text { neurology criteria }\end{array}$ & $\begin{array}{l}\text { Mental disorders: } \\
\text { symptomatic HIV + } \\
5.9 \% \text {, asymptomatic }\end{array}$ & NA & $\begin{array}{l}\text { No specific data for HIV } \\
\text { associated neurocognitive } \\
\text { disorders }\end{array}$ \\
\hline
\end{tabular}

American Academy of

HIV + 1.9\%, HIV- $1.2 \%$ 
Table 3 Overview of studies on HIV-related dementia and risk factors in sub-Saharan (Continued)

\begin{tabular}{|c|c|c|c|c|c|c|c|}
\hline \multirow{5}{*}{$\begin{array}{l}\text { Sacktor [84], } \\
2006\end{array}$} & \multirow{5}{*}{$\begin{array}{l}\text { Uganda, } \\
\text { Hospital }\end{array}$} & Prospective & 23 (men 5) HIV + participants on & \multirow{5}{*}{$\begin{array}{l}\text { MSK HIV dementia } \\
\text { scale IHDS }\end{array}$} & \multirow{3}{*}{$\begin{array}{l}\text { Baseline: Subclinical } \\
\text { dementia 35\% }\end{array}$} & \multirow[t]{5}{*}{ NA } & \multirow{5}{*}{$\begin{array}{l}\text { All participants had CD4 } \\
\text { count } \leq 200 \text { cells } / \mathrm{mL} \text { and } \\
\text { an } I H D S \leq 10 \text { (suggestive } \\
\text { of HAND) }\end{array}$} \\
\hline & & Cohort study & & & & & \\
\hline & & \multirow[t]{3}{*}{$2004-2005$} & HAART (mean age 32.8 y.) & & & & \\
\hline & & & Re-assessment at 3 and 6 months. & & Mild dementia $61 \%$ & & \\
\hline & & & & & $\begin{array}{l}\text { At } 3 \text { (6) months: mild } \\
\text { dementia } 26 \%(4 \%)\end{array}$ & & \\
\hline \multirow{2}{*}{$\begin{array}{l}\text { Sacktor [85], } \\
2005\end{array}$} & \multirow{2}{*}{$\begin{array}{l}\text { Uganda, } \\
\text { Hospital }\end{array}$} & \multirow{2}{*}{$\begin{array}{l}\text { Cross-sectional } \\
2003-2004\end{array}$} & \multirow{2}{*}{$\begin{array}{l}81 \text { HIV+; mean age: } 37 \mathrm{y} ; \\
100 \text { HIV- mean age: } 31.4 \text { y; } \\
21 \text { had HIV dementia }\end{array}$} & IHDS (cut off $\leq 10$ ), & \multirow[t]{2}{*}{ HIV dementia: 31\% } & \multirow[t]{2}{*}{ NA } & \\
\hline & & & & MSK HIV dementia scale & & & \\
\hline \multirow{2}{*}{$\begin{array}{l}\text { Modi [86], } \\
2007\end{array}$} & \multirow{2}{*}{$\begin{array}{l}\text { South-Africa, } \\
\text { Hospital }\end{array}$} & Cross-sectional & \multirow{2}{*}{$\begin{array}{l}506 \text { HIV + (men 203) on HAART; } \\
\text { mean age/range: } 37 \text { years } 193 \\
\text { had HIV associated dementia }\end{array}$} & \multirow{2}{*}{$\begin{array}{l}\text { American Academy of } \\
\text { Neurology AIDS Task force }\end{array}$} & \multirow[t]{2}{*}{ HIV dementia: 38\% } & \multirow[t]{2}{*}{ NA } & \multirow{2}{*}{$\begin{array}{l}75 \% \text { had CD4 below } \\
100 \text { cells/mm3 }\end{array}$} \\
\hline & & 2005 & & & & & \\
\hline \multirow{3}{*}{$\begin{array}{l}\text { Clifford [87], } \\
2007\end{array}$} & \multirow{3}{*}{$\begin{array}{l}\text { Ethiopia, } \\
\text { Hospital }\end{array}$} & Case-control & \multirow{2}{*}{$\begin{array}{l}73 \text { (men 67\%) HIV + participants } \\
\text { (median age } 39 \mathrm{y} \text {.); }\end{array}$} & \multirow[t]{3}{*}{ IHDS } & \multirow[t]{3}{*}{ NA } & \multirow[t]{3}{*}{ NA } & \multirow{3}{*}{$\begin{array}{l}\text { Quantitative } \\
\text { neuropsychiatric } \\
\text { tests - no difference } \\
\text { between groups }\end{array}$} \\
\hline & & 2004 & & & & & \\
\hline & & & $\begin{array}{l}87 \text { (men 63\%) HIV- participants } \\
\text { (median age } 38 \text { y.) }\end{array}$ & & & & \\
\hline \multirow{3}{*}{$\begin{array}{l}\text { Odiase [88], } \\
2007\end{array}$} & \multirow{3}{*}{$\begin{array}{l}\text { Nigeria, } \\
\text { Hospital }\end{array}$} & case-control & \multirow{2}{*}{$\begin{array}{l}96 \text { (men 48) symptomatic HIV + } \\
\text { patients (mean age } 33.6 \text { y.), }\end{array}$} & \multirow{3}{*}{$\begin{array}{l}\text { FePsy computerized } \\
\text { neuropsychological } \\
\text { test battery }\end{array}$} & \multirow[t]{3}{*}{ NA } & \multirow[t]{3}{*}{ NA } & $\begin{array}{l}\text { Severity of immune } \\
\text { suppression predictive }\end{array}$ \\
\hline & & 2004 & & & & & of cognitive decline \\
\hline & & & $\begin{array}{l}\text { (mean age } 31.5 \text { y.); } 96 \text { (men 48) } \\
\text { HIV- (mean age } 32.9 \text { y.) }\end{array}$ & & & & \\
\hline Wong [89], & Uganda, & Cross-sectional & 78 (men 28) HIV + participants & MSK HIV dementia scale & HIV dementia. 31\% & Age, low CD4 & \\
\hline & Hospltal & 2003-2004 & $\begin{array}{l}\text { with dementia; } 100 \text { HIV - } \\
\text { participants }\end{array}$ & & & $\begin{array}{l}\text { Count assoclated } \\
\text { HIV dementia }\end{array}$ & \\
\hline Robertson [90], & Uganda, & Cross-sectional & 110 (men 34) HIV + participants & MSK HIV dementia scale & NA & NA & Pattern of \\
\hline & & 2003-2004 & mean age 36.7 y:; 49 on HAART & & & & deficits similar to that in \\
\hline & & & $\begin{array}{l}100 \text { (men 60) HIV-controls } \\
\text { (mean age } 27.5 \mathrm{y} . \text { ) }\end{array}$ & & & & western countres. \\
\hline $\begin{array}{l}\text { Salawu [91], } \\
2008\end{array}$ & $\begin{array}{l}\text { Nigeria, } \\
\text { hospital }\end{array}$ & Cross-sectional & $\begin{array}{l}60 \mathrm{HIV}+(\text { men 24), asymptomatic, } \\
\text { naïve of HAART; mean age } 32 \mathrm{y} \text { ) }\end{array}$ & CSID & $56.7 \%$ & $\begin{array}{l}\text { No correlation } \\
\text { between CD4 }\end{array}$ & \\
\hline & & & 60 HIV- (men 24); mean age: 30.1 y; & & & performance on & \\
\hline & & & 34 had HIV dementia & & & $\begin{array}{l}\text { neuropsychological } \\
\text { testing }\end{array}$ & \\
\hline $\begin{array}{l}\text { Singh [92], } \\
2008\end{array}$ & $\begin{array}{l}\text { South Africa, } \\
\text { Hospital }\end{array}$ & Cross-sectional & $\begin{array}{l}20 \mathrm{HIV}+\text { (men } 8 \text { ) participants; } \\
\text { median age } 34 \text { y }\end{array}$ & IHDS-criteria (cut-off $\leq 10$ ) & HAND: $80 \%$ & NA & $\begin{array}{l}\text { CD4 < } 200 \text { cells } / \mathrm{mm} 3, \\
\text { older than } 18 \text { years and }\end{array}$ \\
\hline & & 2007 & 16 had HAND & & & & \\
\hline
\end{tabular}


Table 3 Overview of studies on HIV-related dementia and risk factors in sub-Saharan (Continued)

\begin{tabular}{|c|c|c|c|c|c|c|c|}
\hline \multirow[t]{3}{*}{ Säll [93], 2009} & \multirow{3}{*}{$\begin{array}{l}\text { South Africa, } \\
\text { Hospital }\end{array}$} & Retrospective & \multirow{2}{*}{$\begin{array}{l}38 \mathrm{HIV}+\text { admitted to the } \\
\text { psychiatric ward with psychiatric } \\
\text { symptoms; mmean age } 32.4 \mathrm{y}\end{array}$} & \multirow[t]{3}{*}{ DSM-IV } & \multirow[t]{3}{*}{ Dementia: 32\% } & \multirow[t]{3}{*}{ NA } & \\
\hline & & \multirow[t]{2}{*}{ 1987-1997 } & & & & & \\
\hline & & & 12 had dementia & & & & \\
\hline \multirow{2}{*}{$\begin{array}{l}\text { Ganasen [94], } \\
2008\end{array}$} & \multirow{2}{*}{$\begin{array}{l}\text { South Africa, } \\
\text { Hospital }\end{array}$} & \multirow[t]{2}{*}{ Cross-sectional } & \multirow{2}{*}{$\begin{array}{l}474 \text { (men } 123) \text { HIV + patients } \\
\text { ( } 328 \text { blacks and } 135 \text { coloured); } \\
\text { mean age } 34 \mathrm{y} \text {. }\end{array}$} & HIV dementia scale & \multirow{2}{*}{$\begin{array}{l}\text { HAND: } 17.1 \% \text { (IHDS) } \\
\text { and 2.3\% (MMSE) }\end{array}$} & \multirow[t]{2}{*}{ NA } & \\
\hline & & & & MMSE & & & \\
\hline \multirow{3}{*}{$\begin{array}{l}\text { Njamnshi [95], } \\
2008\end{array}$} & \multirow{3}{*}{$\begin{array}{l}\text { Cameroon, } \\
\text { Hospital }\end{array}$} & \multirow{3}{*}{$\begin{array}{l}\text { Case-control } \\
\text { study } 2006\end{array}$} & \multirow{3}{*}{$\begin{array}{l}204 \text { (men 64) HIV + participants } \\
\text { (mean age } 37.2 \text { y.); } 204 \text { (men 64) } \\
\text { HIV- participants (mean age } 37.1 \text { y.) }\end{array}$} & \multirow[t]{3}{*}{ IHDS-criteria (cut-off $\leq 10$ ) } & HAND: & \multirow[t]{3}{*}{ NA } & \\
\hline & & & & & $\mathrm{HIV+:} 21.1 \%$ & & \\
\hline & & & & & HIV-: $2.5 \%$ & & \\
\hline \multirow{5}{*}{$\begin{array}{l}\text { Sacktor [96], } \\
2009\end{array}$} & \multirow{5}{*}{$\begin{array}{l}\text { Uganda, } \\
\text { Hospital }\end{array}$} & \multirow{2}{*}{$\begin{array}{l}\text { Prospective } \\
\text { cohort }\end{array}$} & \multirow{3}{*}{$\begin{array}{l}102 \text { (men 29) HIV + never } \\
\text { treated patients (mean age } \\
34.2 \text { y.) started on Stavudine- } \\
\text { based HAART }\end{array}$} & \multirow{5}{*}{$\begin{array}{l}\text { IHDS criteria } \\
\text { MSK HIV dementia scale }\end{array}$} & Base line: $40 \%$ had & \multirow[t]{5}{*}{ NA } & \\
\hline & & & & & $\begin{array}{l}\text { HIV dementia (33\% } \\
\text { mild, } 7 \% \text { moderate) }\end{array}$ & & \\
\hline & & 2005-2007 & & & & & \\
\hline & & \multirow[t]{2}{*}{$\begin{array}{l}\text { Follow-up } \\
6 \text { months }\end{array}$} & \multirow[t]{2}{*}{$\begin{array}{l}25 \text { (men 15) HIV- } \\
\text { (mean age } 30.3 \text { y.) }\end{array}$} & & $\begin{array}{l}\text { At } 3 \text { months: } 26 \% \text {, } \\
23 \% \text { mild, } 3 \% \\
\text { moderate }\end{array}$ & & \\
\hline & & & & & $\begin{array}{l}\text { At } 6 \text { months: } 16 \% \\
\text { ( } 13 \% \text { mild, } 3 \% \\
\text { moderate }\end{array}$ & & \\
\hline \multirow{2}{*}{$\begin{array}{l}\text { Njamnshi [97], } \\
2009\end{array}$} & \multirow{2}{*}{$\begin{array}{l}\text { Cameroon, } \\
\text { Hospital }\end{array}$} & Cross-sectional & \multirow{2}{*}{$\begin{array}{l}185 \text { (men 61) HIV + participants } \\
\text { (mean age } 37 \text { y.); } 41 \text { with } \\
\text { possible HAND (mean age 37y.) }\end{array}$} & \multirow[t]{2}{*}{ IHDS-criteria } & \multirow[t]{2}{*}{ HAND: $22.2 \%$} & \multirow{2}{*}{$\begin{array}{l}\text { Advanced clinical } \\
\text { stage, low CD4 } \\
\text { count, and low } \\
\text { haemoglobin } \\
\text { levels }\end{array}$} & \\
\hline & & 2006 & & & & & \\
\hline Sacktor [98], & Uganda, & Cross-sectional & $60 \mathrm{HIV}+$ never treated & IHDS criteria & Overall: $36.7 \%$ & HIV subtype D & All participants had CD4, \\
\hline & Hospital & 2005-2007 & dementia & MSK HIV dementia scale & & $\begin{array}{l}\text { increased risk of } \\
\text { HIV dementia }\end{array}$ & $\begin{array}{l}\text { and an IHDS } \leq 10 \\
\text { (suggestive of HAND) }\end{array}$ \\
\hline Nakasujja [99], & Uganda, & Prospective & $102 \mathrm{HIV}+$ (men 28); mean & IHDS (cut-off $\leq 10)$ & Base line: 68.6\% & NA & \\
\hline & Hospital & & impairment at baseline & neuropsychological & At 3 months. $36 \%$ & & \\
\hline & & & & $\begin{array}{l}\text { tests and MSK HIV } \\
\text { dementia scale }\end{array}$ & At 6 months: $30 \%$ & & \\
\hline Kinyanda [100], & Uganda, & Cross-sectional & $618 \mathrm{HIV}+($ men 169), $83 \%<45 y$ & IHDS (cut-off $\leq 10)$ & $64 \%$ & & \\
\hline & Hospital & 2010 & 396 had cognitive disorders & & & & \\
\hline Choi [101], & Guinea Bissau, & Case-control & $22 \mathrm{HIV}-2+$ (men 4)participants & IHDS & HIV+: $22.7 \%$ & age $(\beta=-0.11)$ & \\
\hline & Hospital & & $\begin{array}{l}<350=55.1 \mathrm{y}, \text { mean age for } \\
\text { those with } C D 4 \geq 350=50.3 \mathrm{y} \text { ) }\end{array}$ & & CD4 $\geq 350=18 \%)$ & & \\
\hline & & & $\begin{array}{l}45 \mathrm{HIV} \text { - controls (men 1); mean } \\
\text { age } 51 \cdot 9 \mathrm{y})\end{array}$ & MSK HIV dementia scale & Control: $11 \%$ & & \\
\hline
\end{tabular}


Table 3 Overview of studies on HIV-related dementia and risk factors in sub-Saharan (Continued)

\begin{tabular}{|c|c|c|c|c|c|c|c|}
\hline \multirow{4}{*}{$\begin{array}{l}\text { Birbeck [102], } \\
2011\end{array}$} & Zambia & Cross-sectional & \multirow{2}{*}{$\begin{array}{l}496 \mathrm{HIV}+(\text { men 205) participants } \\
\text { screened within } 1 \text { week of } \\
\text { initiating ART; mean age } 38.1 \text { y) }\end{array}$} & INHDS (cutt-off $\leq 10)$ & $42.1 \%$ (IHDS) & \multirow[t]{4}{*}{ NA } & \multirow{4}{*}{$\begin{array}{l}\text { Low IHDS score was } \\
\text { associated with poor } \\
\text { adherence to HAART }\end{array}$} \\
\hline & \multirow[t]{3}{*}{ Hospital } & \multirow[t]{3}{*}{ 2006-2007 } & & \multirow[t]{3}{*}{ MMSE $(<=22)$} & \multirow[t]{3}{*}{$34.4 \%$ (ZMMSE) } & & \\
\hline & & & $\begin{array}{l}\text { IHDS administered to } 440 \\
\text { participants. }\end{array}$ & & & & \\
\hline & & & 185 had dementia & & & & \\
\hline $\begin{array}{l}\text { Joska [103], } \\
2010\end{array}$ & $\begin{array}{l}\text { South Africa, } \\
\text { Hospital }\end{array}$ & Cross-sectional & $\begin{array}{l}536 \text { (men } 26.7 \%) \text { HIV }+ \\
\text { participants (68\% blacks, } \\
28 \% \text { coloured), mean age } 34 \text { y. }\end{array}$ & HDS (cutt-off $\leq 10)$ & HAND: $23.5 \%$ & $\begin{array}{l}\text { Age, education, } \\
\text { diagnosed duration, } \\
\text { post-traumatic } \\
\text { stress disorder }\end{array}$ & $\begin{array}{l}\text { IDHS not yet available by } \\
\text { the time of the study }\end{array}$ \\
\hline \multirow{2}{*}{$\begin{array}{l}\text { Kanmogne } \\
\text { [104], } 2010\end{array}$} & Cameroon & Case-control & \multirow{2}{*}{$\begin{array}{l}43 \text { (men 18) HIV- participants } \\
\text { (mean age } 33.3 \text { y.); } 44 \text { (men 17) } \\
\text { HIV + participants (mean age } \\
34.9 \text { y.); } 22 \text { with AIDs defining } \\
\text { conditions, } 34 \% \text { on HAART }\end{array}$} & \multirow{2}{*}{$\begin{array}{l}\text { HIV Neurobehavioral } \\
\text { Research Center } \\
\text { International } \\
\text { neuropsychological } \\
\text { test battery }\end{array}$} & \multirow[t]{2}{*}{ NA } & \multirow[t]{2}{*}{ NA } & \\
\hline & Hospital & 2008-2009 & & & & & \\
\hline \multirow[t]{2}{*}{$\begin{array}{l}\text { Lawler [105], } \\
2010\end{array}$} & Botswana, & Cross-sectional & $\begin{array}{l}120 \text { (men 60) HIV + patients } \\
\text { (mean age } 37.5 \text { y.); } 97.5 \% \\
\text { on HAART; }\end{array}$ & \multirow[t]{2}{*}{ IHDS-criteria (cut-off $\leq 9.5$ ) } & \multirow[t]{2}{*}{ HAND: 38\% } & \multirow[t]{2}{*}{ NA } & \\
\hline & Hospital & 2008 & 46 with HIV dementia & & & & \\
\hline \multirow{5}{*}{$\begin{array}{l}\text { Patel [106], } \\
2010\end{array}$} & \multirow{5}{*}{$\begin{array}{l}\text { Malawi, } \\
\text { Hospital }\end{array}$} & Cross sectional & \multirow{2}{*}{$\begin{array}{l}179 \text { (men 63) HIV + participants } \\
\text { (mean age } 36.7 \text { y.); Stage III/IV } \\
\text { 90\%; } 134 \text { on HAART > } 6 \text { months; }\end{array}$} & \multirow[t]{5}{*}{ IHDS-criteria (cut-off $\leq 10)$} & HAD & \multirow{5}{*}{$\begin{array}{l}\text { Female gender, } \\
\text { low education }\end{array}$} & \\
\hline & & 2007 & & & & & \\
\hline & & & \multirow[t]{3}{*}{25 (men 14) with HIV dementia } & & Overall: $14 \%$ & & \\
\hline & & & & & Men: $22.2 \%$ & & \\
\hline & & & & & Women: 9.5\% & & \\
\hline \multirow{2}{*}{$\begin{array}{l}\text { Siddiqi [69], } \\
2009\end{array}$} & Zambia & Cross-sectional & \multirow{2}{*}{$\begin{array}{l}443 \text { (men 219) inpatients } \\
\text { (median age } 39 \text { y., } 67 \mathrm{HIV}+\text { ); } \\
368 \text { (men 168) outpatients } \\
\text { (median age } 39 \text { y., } 58 \mathrm{HIV}+\text { ); } \\
\text { Overall } 36 \text { cases of dementia }\end{array}$} & \multirow[t]{2}{*}{ Not specified } & \multirow[t]{2}{*}{ NA } & HIV+: $10.4 \%$ & \multirow{7}{*}{$\begin{array}{l}\text { HIV + patient had a } \\
\text { higher frequency of } \\
\text { dementia and had } \\
\text { dementia at } \\
\text { younger age }\end{array}$} \\
\hline & Hospital & & & & & HIV-: $3.3 \%$ & \\
\hline \multirow{2}{*}{$\begin{array}{l}\text { Ekenze [21], } \\
2010\end{array}$} & \multirow{2}{*}{$\begin{array}{l}\text { Nigeria, } \\
\text { Hospital }\end{array}$} & Cross-sectional & \multirow{2}{*}{$\begin{array}{l}8440 \text { admissions; } 1249 \\
\text { (men 640) with neurological } \\
\text { diseases (mean age } 45 \text { y.); } \\
44 \text { (men 18) with AIDS } \\
\text { dementia complex }\end{array}$} & \multirow[t]{2}{*}{ Not specified } & \multirow{2}{*}{$\begin{array}{l}\text { AIDS dementia } \\
\text { complex: } 3.5 \% \text { of } \\
\text { all neurological } \\
\text { admission }\end{array}$} & \multirow[t]{2}{*}{ NA } & \\
\hline & & 2003-2007 & & & & & \\
\hline \multirow{3}{*}{$\begin{array}{l}\text { Holguin [107], } \\
2011\end{array}$} & Zambia, & Case-control & 57 (men 30) HIV-participants & IHDS (cut-off $\leq 10)$ & HAND = 22\% & NA & \\
\hline & & & HIV + (mean age 34 y.) & Color Trails Test 1 and & naïve of ARV & & \\
\hline & & 2008 & including 54 naïve of HAART & $\begin{array}{l}\text { 2, Grooved pegboard Test, } \\
\text { and Time Gait Test }\end{array}$ & & & \\
\hline
\end{tabular}


Table 3 Overview of studies on HIV-related dementia and risk factors in sub-Saharan (Continued)

\begin{tabular}{|c|c|c|c|c|c|c|c|}
\hline $\begin{array}{l}\text { Joska [108], } \\
2011\end{array}$ & $\begin{array}{l}\text { South Africa, } \\
\text { Hospital }\end{array}$ & $\begin{array}{l}\text { Case-control } \\
2008\end{array}$ & $\begin{array}{l}94 \text { (men 36) HIV- participants } \\
\text { (mean age } 25.2 \text { y); } 96 \text { (men 20) } \\
\text { HIV + (mean age } 29.8 \text { y) }\end{array}$ & IHDS & NA & $\begin{array}{l}\text { Education associated } \\
\text { with } \mathrm{HDS} \text { total score }\end{array}$ & $\begin{array}{l}\text { Validation study of } \\
\text { the IHDS }\end{array}$ \\
\hline $\begin{array}{l}\text { Obiabo [109], } \\
2011\end{array}$ & $\begin{array}{l}\text { Nigeria, } \\
\text { Hospital }\end{array}$ & $\begin{array}{l}\text { Prospective } \\
\text { Cohort study }\end{array}$ & $\begin{array}{l}69 \text { (men 25) HIV + participants } \\
\text { with CD4 < } 350 \text { (mean age } \\
36.2 \text { y.); } 30 \text { (men 11) HIV- } \\
\text { (mean age } 36.6 \text { y.) }\end{array}$ & $\begin{array}{l}\text { CSID and FePsy } \\
\text { computerized } \\
\text { neuropsychological } \\
\text { test battery }\end{array}$ & NA & NA & $\begin{array}{l}\text { HAART improved } \\
\text { neuropsychological } \\
\text { performances after } \\
12 \text { months of treatment }\end{array}$ \\
\hline $\begin{array}{l}\text { Joska [110], } \\
2011\end{array}$ & $\begin{array}{l}\text { South Africa } \\
\text { Hospital }\end{array}$ & $\begin{array}{l}\text { Cross-sectional } \\
2008-2009\end{array}$ & $\begin{array}{l}170 \text { (men 44) HIV + participants } \\
\text { (mean age } 29.5 \text { y.)never treated; } \\
43 \text { (men 14) with HIV-dementia; } \\
72 \text { (men } 19 \text { with MND }\end{array}$ & AAN revised criteria & $\begin{array}{l}\text { Mild neurocognitive } \\
\text { disorder: } 42.4 \% \text { HIV } \\
\text { dementia: } 25.4 \%\end{array}$ & $\begin{array}{l}\text { Education, and male } \\
\text { gender independent } \\
\text { predictors of } \\
\text { HIV-dementia }\end{array}$ & \\
\hline $\begin{array}{l}\text { Robertson } \\
{[111], 2011}\end{array}$ & $\begin{array}{l}\text { Malawi, } \\
\text { Hospital }\end{array}$ & Cross sectional & $\begin{array}{l}133 \text { (men 39) never treated } \\
\text { HIV + patients (median } \\
\text { age } 31 \text { y.) }\end{array}$ & Not provided & $\begin{array}{l}\text { MND: } 8 \% \\
\text { HAD: } 0 \%\end{array}$ & & \\
\hline & $\begin{array}{l}\text { South Africa, } \\
\text { Hospital }\end{array}$ & & $\begin{array}{l}167 \text { (men 60) never treated } \\
\text { HIV + patients (median } \\
\text { age } 34 \text { y.) }\end{array}$ & Not provided & $\begin{array}{l}\text { MND: } 4 \% \\
\text { HAD: } 0 \%\end{array}$ & & \\
\hline & $\begin{array}{l}\text { Zimbabwe, } \\
\text { Hospital }\end{array}$ & & $\begin{array}{l}80 \text { (men 31) never treated } \\
\text { HIV + patients (median } \\
\text { age } 36 \mathrm{y} \text {.) }\end{array}$ & Not provided & $\begin{array}{l}\text { MND: } 14 \% \\
\text { HAD: } 3 \%\end{array}$ & NA & $\begin{array}{l}860 \mathrm{HIV}+\mathrm{HAART} \text { naïve } \\
\text { patients with CD4 count } \\
<300 \text { cells/mL and } \mathrm{KI} \geq 70 \%\end{array}$ \\
\hline $\begin{array}{l}\text { Robbins [112], } \\
2011\end{array}$ & $\begin{array}{l}\text { South Africa, } \\
\text { Hospital }\end{array}$ & $\begin{array}{l}\text { Cross-sectional } \\
2009-2010\end{array}$ & $\begin{array}{l}65 \text { (men 23) HIV + patients } \\
\text { on HAART for } \geq 6 \text { months } \\
\text { (mean age } 38.5 \mathrm{y} \text { ) }\end{array}$ & $\begin{array}{l}\text { IHDS and Xhosa-validated } \\
\text { IHDS }\end{array}$ & $\begin{array}{l}\text { HIV Associated } \\
\text { dementia } 80 \%\end{array}$ & $\begin{array}{l}\text { Low CD4 } \\
\text { counts, alcohol } \\
\text { dependency }\end{array}$ & \\
\hline $\begin{array}{l}\text { Kwasa [113], } \\
2012\end{array}$ & $\begin{array}{l}\text { Kenya, } \\
\text { Hospital }\end{array}$ & Cross sectional & $\begin{array}{l}30 \text { (men 17) HIV + patients } \\
\text { (mean age } 39 \mathrm{y} \text {.) } \\
6 \text { (men 5) with HAD }\end{array}$ & $\begin{array}{l}\text { Neuropsychological test } \\
\text { battery MMSE/IHDS } \\
\text { (cut-off } \leq 10 \text { ) }\end{array}$ & HAD 20\% & NA & \\
\hline $\begin{array}{l}\text { Spies [114], } \\
2012\end{array}$ & $\begin{array}{l}\text { South-Africa, } \\
\text { Hospital }\end{array}$ & Case-control & $\begin{array}{l}35 \mathrm{HIV}+\text { without childhood } \\
\text { trauma; mean age: } 31.5 \text { y } \\
48 \mathrm{HIV}+\text { with childhood } \\
\text { trauma; mean age: } 31.7 \text { y } \\
27 \mathrm{HIV} \text { - without childhood } \\
\text { trauma; mean: } 25 y \\
20 \mathrm{HIV} \text { - with childhood } \\
\text { trauma; mean age: } 27 \cdot 7 \text { y } \\
\text { All participants were women. }\end{array}$ & $\begin{array}{l}\text { Neuropsychological } \\
\text { test battery }\end{array}$ & NA & NA & $\begin{array}{l}\text { Significant HIV effects for } \\
\text { the Hopkins Verbal } \\
\text { Learning Test (HVLT) } \\
\text { learning and delay trials } \\
\text { and the Halstead } \\
\text { Category Test }(H C T)\end{array}$ \\
\hline $\begin{array}{l}\text { Hestad [115], } \\
2012\end{array}$ & $\begin{array}{l}\text { Zambia, } \\
\text { Hospital }\end{array}$ & Case-control & $\begin{array}{l}38 \mathrm{HIV}+(\text { men } 16) ; \text { mean } \\
\text { age: } 28.3 \text { y } 42 \mathrm{HIV} \text { - (men 18); } \\
\text { mean age: } 28.9 \text { y }\end{array}$ & Neuropsychological tests & NA & NA & $\begin{array}{l}\text { HIV + individuals } \\
\text { performance lower than } \\
\text { that of HIV- on verbal } \\
\text { fluency, executive } \\
\text { function, speed of } \\
\text { information processing, } \\
\text { verbal episodic memory } \\
\text { and motor function }\end{array}$ \\
\hline
\end{tabular}


Table 3 Overview of studies on HIV-related dementia and risk factors in sub-Saharan (Continued)

\begin{tabular}{|c|c|c|c|c|c|c|c|}
\hline \multirow{3}{*}{$\begin{array}{l}\text { Berhe [116], } \\
2012\end{array}$} & Ethiopia, & Cross-sectional & \multirow{2}{*}{$\begin{array}{l}347 \text { HIV + (men 176) participants; } \\
\text { mean age/range: } 34.6 \text { y admitted } \\
\text { with neurological disorders }\end{array}$} & \multirow{3}{*}{$\begin{array}{l}\text { "cognitive and motor } \\
\text { abnormalities, CT/MRI } \\
\text { showing brain atrophy } \\
\text { and other opportunistic } \\
\text { infections ruled out" }\end{array}$} & \multirow{3}{*}{$\begin{array}{l}\text { HIV encephalopathy: } \\
0.3 \%\end{array}$} & \multirow[t]{3}{*}{ NA } & \\
\hline & \multirow[t]{2}{*}{ Hospital } & Retrospective & & & & & \\
\hline & & 2002-2009 & 10 had dementia & & & & \\
\hline \multirow{2}{*}{$\begin{array}{l}\text { Joska [117], } \\
2012\end{array}$} & South Africa, & \multirow[t]{2}{*}{ Prospective } & \multirow{2}{*}{$\begin{array}{l}166 \mathrm{HIV}+\text { participants assessed } \\
\text { at baseline, } 108 \text { reassessed at } \\
\text { one year ( } 82 \text { received HAART) }\end{array}$} & Neuropsychological tests & \multirow[t]{2}{*}{ NA } & \multirow{2}{*}{$\begin{array}{l}\text { Lower level of } \\
\text { education }\end{array}$} & \multirow{13}{*}{$\begin{array}{l}\text { Improvement on } \\
\text { neuropsychological } \\
\text { tests for all participants } \\
\text { at one year. }\end{array}$} \\
\hline & Hospital & & & $\begin{array}{l}\text { Average Global } \\
\text { deficit score }\end{array}$ & & & \\
\hline \multirow{2}{*}{$\begin{array}{l}\text { Breuer [118], } \\
2012\end{array}$} & South Africa, & \multirow[t]{2}{*}{ Cross-sectional } & \multirow{2}{*}{$\begin{array}{l}269 \text { HIV + (men 97) participants } \\
\text { on HAART for } \geq 6 \text {; months; } 34 \% \\
\text { aged }>40 \text { y) }\end{array}$} & \multirow[t]{2}{*}{ IHDS (cut-off $\leq 10.5$ ) } & \multirow[t]{2}{*}{ HAND: $12 \%$} & \multirow[t]{2}{*}{ NA } & \\
\hline & Hospital & & & & & & \\
\hline \multirow[t]{2}{*}{$\begin{array}{l}\text { Hoare [119], } \\
2012\end{array}$} & South Africa & \multirow[t]{2}{*}{ Cross-sectional } & \multirow[t]{2}{*}{$\begin{array}{l}43 \text { stage III HIV + ( } 24 \text { with at least } \\
\text { one } \varepsilon 4 \text { ApoE allele, men: } 8 \text {, Age: } \\
29 \text { y and } 19 \text { without the } \varepsilon 4 \text { ApoE } \\
\text { allele, men: } 2 \text {, Age: } 28 \text { y) }\end{array}$} & \multirow[t]{2}{*}{$\begin{array}{l}\text { Neuropsychological } \\
\text { test battery }\end{array}$} & \multirow[t]{2}{*}{ NA } & $\begin{array}{l}\text { Performance on } \\
\text { Hodgkin Verbal } \\
\text { Learning Tool- } \\
\text { Revised was poorer } \\
\text { in the group with } \\
\text { the } \varepsilon 4 \text { genotype. }\end{array}$ & \\
\hline & Hospital & & & & & $\begin{array}{l}\text { Participants with } \\
\text { the } \varepsilon 4 \text { genotype } \\
\text { had more white } \\
\text { matter injury } \\
\text { on MRI. }\end{array}$ & \\
\hline \multirow{5}{*}{$\begin{array}{l}\text { Oshinaike } \\
\text { [120], } 2012\end{array}$} & Nigeria & Case-control & \multirow{2}{*}{$\begin{array}{l}208 \text { HIV + (men } 71) \\
\text { mean age: } 36.8 \text { y }\end{array}$} & \multirow[t]{2}{*}{ IHDS (cut off $\leq 10$ ) } & \multirow[t]{3}{*}{ HAND by MMSE: $2.9 \%$} & \multirow[t]{5}{*}{ Lower CD4 count } & \\
\hline & Hospital & $2007-2008$ & & & & & \\
\hline & & & \multirow{3}{*}{$\begin{array}{l}121 \text { HIV - (men: 35), } \\
\text { mean age:38.0 y }\end{array}$} & MMSE (cut off $\leq 26$ ) & & & \\
\hline & & & & \multirow{2}{*}{$\begin{array}{l}\text { AAN revised criteria } \\
\text { (any value below 2SD) }\end{array}$} & HAND by IHDS: $54.3 \%$ & & \\
\hline & & & & & HAND by AAN: $42.3 \%$ & & \\
\hline \multirow[t]{2}{*}{$\begin{array}{l}\text { Royal [121], } \\
2012\end{array}$} & $\begin{array}{l}\text { Nigeria, } \\
\text { Hospital }\end{array}$ & Cross-sectional & $\begin{array}{l}60 \text { (men 23) never } \\
\text { treated HIV + participants } \\
\text { (mean age } 34 \text { y); }\end{array}$ & IHDS (cut off $\leq 10$ ) & $\begin{array}{l}28.8 \% \text { HIV + individuals } \\
\text { scored abnormally }\end{array}$ & \multirow[t]{2}{*}{$\begin{array}{l}\text { Low CD4 count, } \\
\text { WHO clinical stage } \\
\text { of disease }\end{array}$} & \\
\hline & & & $\begin{array}{l}56 \text { (men 34) HIV- } \\
\text { (mean age } 29 \cdot 4 \text { y.); } \\
32 \text { had dementia }\end{array}$ & & $\begin{array}{l}16.0 \% \text { HIV- individuals } \\
\text { scored abnormally }\end{array}$ & & \\
\hline
\end{tabular}

3TC: Lamivudine; AIDS: Acquired Immunodeficiency Syndrome; CD4: cluster of differentiation 4; CSID: Community Screening Interview for Dementia; CT: computerized tomography; DSM-III-R: Diagnostic and Statistical Manual $3^{\text {rd }}$ edition revised; DSM-IV: Diagnostic and Statistical Manual $4^{\text {th }}$ edition; dT4: Didanosine; FePsy: The lon Psyche Program; HAART: Highly Active Anti-Retroviral Treatment; HAD: HIV Associated Dementia; HAND: HIV Associated Neurocognitive Disorders; HDS: HIV Dementia Scale; HIV: Human Immunodeficiency Virus; ICD-III-R: International Classification of Disease; IHDS: International HIV Dementia Scale; MSK: Memorial Sloan Kettering; MMSE: Mini Mental State Examination; MND: Mild Neurocognitive Disorder; NA: Not available; NVP: Nevirapine; WHO: World Health Organization; y, years; ZDV: Zidovudine. 
Table 4 Overview of studies on amyotrophic lateral sclerosis risk factors in sub-Sahara Africa

\begin{tabular}{|c|c|c|c|c|c|c|c|}
\hline $\begin{array}{l}\text { Author, year } \\
\text { of publication }\end{array}$ & Country/setting & Design/year & Population characteristics & Diagnostic criteria/tools & Prevalence & Risk factors & Comments \\
\hline \multirow[t]{2}{*}{ Wall [130],1972 } & Zimbabwe & Retrospective & \multirow{2}{*}{$\begin{array}{l}13 \text { (men 10) consecutive } \\
\text { patients; age } 24-55 \mathrm{y} \text {. }\end{array}$} & \multirow[t]{2}{*}{ Clinical (no ENMG) } & \multirow[t]{2}{*}{ NA } & \multirow[t]{2}{*}{ NA } & \multirow{2}{*}{$\begin{array}{l}6 \text { participants had sensory } \\
\text { changes }\end{array}$} \\
\hline & Hospital-based & 1967-1971 & & & & & \\
\hline \multirow{3}{*}{$\begin{array}{l}\text { Osuntokun [126], } \\
1974\end{array}$} & Nigeria & Retrospective & \multirow{3}{*}{$\begin{array}{l}92 \text { patients with MND ALS 73; } \\
\text { PMA 10, SMA } 9\end{array}$} & \multirow[t]{3}{*}{ ENMG/Muscle biopsy/ } & \multirow[t]{3}{*}{$21 / 100,000$} & \multirow[t]{3}{*}{ NA } & Mean age at onset: 39 y \\
\hline & \multirow[t]{2}{*}{ Hospital-based } & $1958-1973$ & & & & & $\begin{array}{l}\text { Mean duration of disease } \\
\text { exceeded } 15 \text { y in } 8 \% \text { of } \\
\text { participants }\end{array}$ \\
\hline & & & & & & & $\begin{array}{l}4 \text { patients with ALS had } \\
\text { poliomyelitis in childhood. }\end{array}$ \\
\hline \multirow{2}{*}{$\begin{array}{l}\text { Osuntokun [19], } \\
1987\end{array}$} & Nigeria & Cross-sectional & \multirow{2}{*}{$\begin{array}{l}18954 \text { participants (men 9282); } \\
58 \%<20 \text { y and } 11 \%>50 \text { y }\end{array}$} & \multirow{2}{*}{$\begin{array}{l}\text { Screening questionnaire } \\
\text { developed by the authors }\end{array}$} & \multirow[t]{2}{*}{ MND: 15/100,000 } & \multirow[t]{2}{*}{ NA } & \\
\hline & Community-based & 1985 & & & & & \\
\hline \multirow[t]{2}{*}{ Cosnett [125], 1989} & \multirow[t]{2}{*}{$\begin{array}{l}\text { South Africa } \\
\text { Hospital-based }\end{array}$} & \multirow[t]{2}{*}{$\begin{array}{l}\text { Retrospective } \\
\text { Cases collected } \\
\text { during } 9.5 \mathrm{y} \text {. }\end{array}$} & \multirow{2}{*}{$\begin{array}{l}59 \text { blacks (mean age } 47.4 \mathrm{y} \text {.); } \\
16 \text { whites and } 2 \text { coloured } \\
\text { (mean age } 54 \mathrm{y} .) 9 \text { Indians } \\
\text { (mean age } 54 \mathrm{y} \text { ) }\end{array}$} & \multirow[t]{2}{*}{ Clinical and ENMG in 45\% } & \multirow{2}{*}{$\begin{array}{l}\text { Blacks/white \& } \\
\text { coloured/Indians } \\
\text { (per 100,000) } \\
0.88 / 2 \cdot 7 / 1.4\end{array}$} & \multirow[t]{2}{*}{ NA } & $\begin{array}{l}\text { Mean age of onset: } 47 \mathrm{y} \\
\text { (blacks) and } 54 \mathrm{y} \text { (in whites } \\
\text { and Indians) }\end{array}$ \\
\hline & & & & & & & $\begin{array}{l}29 \% \text { of participants not } \\
\text { followed up. }\end{array}$ \\
\hline \multirow[t]{2}{*}{ Ekenze [21], 2010} & Nigeria & Retrospective & \multirow{2}{*}{$\begin{array}{l}8440 \text { admissions; } 1249 \\
\text { (men 640) with neurological } \\
\text { diseases, mean age } 45 \text { y:; } 10 \\
\text { (men 4) with ALS }\end{array}$} & \multirow[t]{2}{*}{ Not specified } & \multirow[t]{2}{*}{$800 / 100,000$} & \multirow[t]{2}{*}{ NA } & \\
\hline & Hospital-based & 2003-2007 & & & & & \\
\hline \multirow[t]{2}{*}{ Abdulla [127], 1997} & Sudan & Retrospective: & \multirow{2}{*}{$\begin{array}{l}28 \text { (men 17) patients with } \\
\text { MND; } 19 \text { (men 14) with ALS }\end{array}$} & \multirow[t]{2}{*}{ Clinical and ENMG } & \multirow[t]{2}{*}{ NA } & Family history & Mean age of onset: $40 \mathrm{y}$ \\
\hline & Hospital-based & 1993-1995 & & & & of MND in $14 \%$ & \\
\hline Kengne [16], 2006 & Cameroon & Retrospective & 4041 neurologic consultations; & Not provided & $12 \%$ of all & & 4 selected degenerative brain \\
\hline & Hospital-based & 1993-2001 & $\begin{array}{l}145 \text { with neurodegenerative } \\
\text { diseases } 10 \text { (men 8) with ALS; } \\
\text { mean age } 50.9 \text { y. }\end{array}$ & & $\begin{array}{l}\text { neurodegeneration } \\
250 / 100,000 \text { of all } \\
\text { neurologic } \\
\text { consultation }\end{array}$ & & $\begin{array}{l}\text { diseases: Dementia, PD, ALS } \\
\text { and chorea }\end{array}$ \\
\hline Imam [131], 2004 & Nigeria & Retrospective & 16 (men 15) participants; & El Escorial diagnostic & NA & NA & \\
\hline & Hospital-based & 1980-99 & & & & & \\
\hline Adam [129], 1992 & Kenya & Retrospective & $\begin{array}{l}47 \text { (men 35) participants } \\
\text { with MND; }\end{array}$ & $\begin{array}{l}\text { Clinical (ENMG in 1/3 } \\
\text { of participants) }\end{array}$ & NA & NA & Duration of disease: $5 \mathrm{~m}$ to $4 \mathrm{y}$. \\
\hline & Hospital-based & 1978-88 & Age $13-80$ y & & & & \\
\hline & & & 18 had ALS & & & & \\
\hline $\begin{array}{l}\text { Tekle-Haimanot [122], } \\
1990\end{array}$ & Ethiopia & Cross-sectional & $\begin{array}{l}60820 \text { participants (men 29412), } \\
59 \% \text { aged }<20 \text { y }\end{array}$ & $\begin{array}{l}\text { Screening questionnaire } \\
\text { and neurological exam }\end{array}$ & $5 / 100,000$ & NA & $\begin{array}{l}\text { A population survey of } \\
\text { neurological diseases }\end{array}$ \\
\hline & Community-based & 1986-88 & 3 (2 men) had MND & & & & \\
\hline Harries [132], 1955 & Ethiopia & Case series & 2(all males) participants & Clinical (no ENMG) & NA & NA & \\
\hline & Hospital-based & 1954 & Age 26 and $30 y$ & & & & \\
\hline
\end{tabular}


Table 4 Overview of studies on amyotrophic lateral sclerosis risk factors in sub-Sahara Africa (Continued)

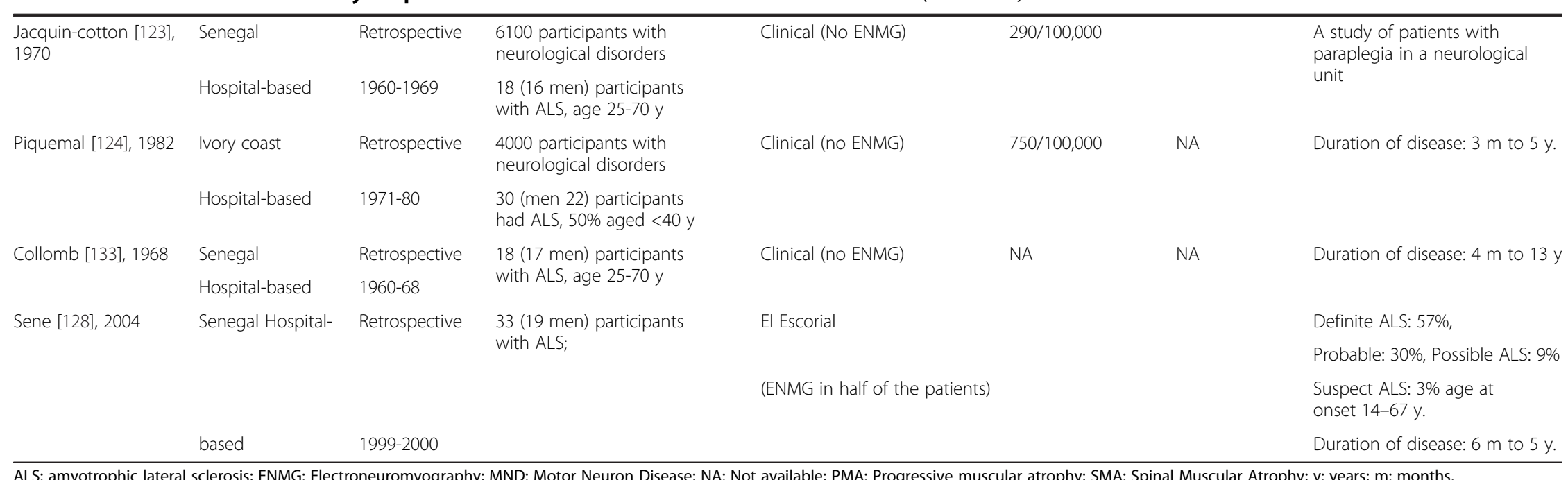

ALS: amyotrophic lateral sclerosis; ENMG: Electroneuromyography; MND: Motor Neuron Disease; NA: Not available; PMA: Progressive muscular atrophy; SMA: Spinal Muscular Atrophy; y: years; m: months. 
eight men, age at onset 12-49 years) having type 2 spino-cerebellar ataxia [134]. A study in Mauritania reported on 12 cases of cerebellar degeneration-based on clinical criteria, including 9 familial cases (including 7 men, aged 3 to 29 years) and 3 apparently sporadic cases (all men, aged 8 to 50 years) [135]. Another clinic-based study of paraplegia in Senegal reported on 7 cases of spino-cerebellar degeneration among 6100 neurological admissions [123].

\section{Huntington disease}

Nineteen studies (four community-based studies and 15 hospital-based) investigated Huntington disease; including 8 cross-sectional studies (including reviews of medical records), 10 case series (two to 13 patients), and one case report (Table 5). The studies were conducted in nine countries: South Africa (nine studies), Zimbabwe and Tanzania (two studies each), Nigeria, Mauritius Island, Senegal, Sudan, Togo and Burkina Faso (one study each). The diagnostic of Huntington disease was mostly clinical, based on a constellation of probing clinical elements; however genetic testing was carried out in five studies [136-140]. The absolute number of participants with Huntington disease ranged from one to 481 . Only one community-based study provided a prevalence estimate of 3.5/100,000 in South-Africa [141]. The hospital-based prevalence of Huntington disease when reported ranged from $0.2 / 100,000$ to 46.0 / $100,000[138,142-146]$. No study reported data on the incidence of Huntington disease. Among those with the disease, males represented 42 to $100 \%$, and age varied from $<9$ years to 80 years. When provided, the age at the clinical onset of the disease ranged from less than one year to 58 years. In general, antecedent risk factors for Huntington disease were not investigated across studies except for a positive family history reported in 58.3 to $100 \%$ of cases.

\section{Discussion}

This review represents an unprecedented effort to summarize epidemiological data on neurodegenerative diseases in SSA. However, this being a large diverse multicultural and multiethnic region, it is difficult to reliably quantify and compare the burden of neurodegenerative disorders across countries. Although mostly based on prevalent cases and on retrospective data, from studies that have essentially included urban populations, findings summarized in the current review are very informative.

The most widely investigated and prevalent neurodegenerative condition appeared to be dementia with most cases being of Alzheimer disease type. Major risk factors of $\mathrm{AD}$ include an advanced age (higher after the age of 60), female sex, a low schooling (less than 6 year of education), family background and rural residence. Unlike North America, Australia, Europe, and Japan where several population-based studies have been conducted on dementia, good quality epidemiological studies (prospective, population-based, using standardized criteria) are scanty in SSA, with methodological issues hampering any meaningful comparison with other regions of the world. The reported prevalence in one collaborative good quality study in Nigeria about 20 years ago among those aged $>60$ years was $2.3 \%$. This was lower than the reported prevalence in developing countries, but within the range of reports from developing countries in Asia and Latin America where reported prevalence range from 1.9 to $3.8 \%$ [155]. The anticipated ageing of the population (which is the main driver of dementia figures) in Africa may translate in a higher prevalence and absolute number of people living with dementia as observed in other developing regions. However, caution is needed when interpreting findings from studies conducted in different settings by different investigators. Our overview tends to suggest that the projected increase in the prevalence of dementia in SSA is likely, based on the comparison of findings from three recent studies with those from the study above conducted in Nigeria 20 years ago [55-57]. Furthermore, with the large scale implementation of antiretroviral therapy and related improved survival, it is expected that the number of patients with the diagnosis of HIV-related neurocognitive impairment may increase as suggested by the increasing number of related-publications. Such trends will need to be confirmed by large scale prospective observational studies which will also assess the putative accelerating effect of HIV-related neurocognitive impairment on other types of prevalent dementia and neurodegeneration.

For Parkinsonism, the wide prevalence range observed both in population and hospital-based studies might also be a consequence of differences in methodologies for case ascertainment, diagnostic criteria, or age distributions of the study populations. These heterogeneities in PD prevalence are not unique to SSA as these have also been observed in Europe where prevalence of PD ranged from 66 to 12,500/100,000 [156]. There have been provisional set of minimal scientific criteria for conducting epidemiological studies on PD which, when adopted at a large scale will improve comparison within SSA and between SSA and other regions of the world [156]. Prevalence rates reported in population-based studies in the continent are limited to two studies and cases were ascertained through screening and neurological exam in one study, thus making any comparison with other region difficult. In ALS and Huntington disease, the picture is less clear as the majority of studies were hospitalbased, retrospective in nature, with a final diagnosis not always based on pathology or genetics and the risk 
Table 5 Overview of studies on Huntington disease and risk factors in sub-Sahara African countries

\begin{tabular}{|c|c|c|c|c|c|c|}
\hline Author, year of publication & Country & Setting & $\begin{array}{l}\text { Design/year of } \\
\text { the study }\end{array}$ & Population characteristics & Diagnostic tool/criteria & Prevalence \\
\hline Hayden [141], 1977 & South Africa & Community & Cross-sectional & 26 cases (men 11); age 12-68 y. & Clinical & $3.5 / 100,000$ \\
\hline \multirow[t]{2}{*}{ Samuels [147], 1978} & \multirow[t]{2}{*}{ Zimbabwe } & \multirow[t]{2}{*}{ Community } & \multirow[t]{2}{*}{ Case series } & 1 family of HD & \multirow[t]{2}{*}{ Clinical } & \multirow[t]{2}{*}{ NA } \\
\hline & & & & 4 cases (men 2) age $14-26 y$. & & \\
\hline Glass [148], 1979 & South Africa & Community & Case series & 2 cases of HD (men 1) age 42-52 & Clinical & NA \\
\hline Hayden [142], 1980 & South Africa & Community/hospital & Cross-sectional, & $\begin{array}{l}481 \text { cases ( } m \text { en } 241 \text { ) of whom } \\
153 \text { ( } m \text { en } 69 \text { ) alive by the time } \\
\text { of the study }\end{array}$ & Clinical & $\begin{array}{l}\text { Overall: } 0.65 / 100,000, \\
\text { Whites: } 2.22 / 100,000, \\
\text { Mixed ancestry: } \\
2.17 / 100,000, \text { Blacks: } \\
0 \cdot 01 / 100,000\end{array}$ \\
\hline Scrimgeour [149], 1981 & Tanzania & Community & Case series & 11 cases, aged $25-80 \mathrm{y}$. & Clinical & NA \\
\hline Hayden [143], 1981 & Mauritius & Hospital & Cross-sectional & 2166 persons, 6 cases of HD (men 3) & Not provided & $46 / 100,000$ \\
\hline \multirow[t]{4}{*}{ Hayden [144], 1981} & \multirow[t]{4}{*}{ South Africa } & \multirow[t]{4}{*}{ Hospital } & \multirow[t]{4}{*}{ Cross-sectional/NR } & \multirow{4}{*}{$\begin{array}{l}17 \text { children (onset before } 20 \mathrm{y} \text {.) } \\
\text { identified during a national survey } \\
\text { among of } 219 \text { patients }\end{array}$} & \multirow[t]{4}{*}{ Not provided } & Overall: 0.6/100,000 \\
\hline & & & & & & Whites: $0.37 / 100,000$ \\
\hline & & & & & & $\begin{array}{l}\text { Mixed ancestry: } \\
0.89 / 100,000\end{array}$ \\
\hline & & & & & & Blacks: No case \\
\hline Hayden [150], 1982 & South Africa & Community/hospital & Cross-sectional & $\begin{array}{l}157 \text { (men } 71 \text { ) individuals investigated } \\
\text { and } 328 \text { (women 156, only } 3 \text { negro-Africans) } \\
\text { deceased individuals with probably HD }\end{array}$ & Not specified & $\begin{array}{l}\text { Combined white and black } \\
\text { heterozygote frequency }= \\
6 \cdot 7 \times 100,000\end{array}$ \\
\hline \multirow[t]{2}{*}{ Scrimgeour [151], 1982} & \multirow[t]{2}{*}{ Tanzania } & \multirow[t]{2}{*}{ Hospital } & \multirow[t]{2}{*}{$\begin{array}{l}\text { Case series } \\
\text { (National registry) }\end{array}$} & $\begin{array}{l}7 \text { patients with chorea }(1 \text { aged } 80 \text { y.) and } \\
50 \text { potential patients with chorea in } \\
23 \text { families }\end{array}$ & \multirow[t]{2}{*}{ Not specified } & \multirow[t]{2}{*}{ NA } \\
\hline & & & & Mean age at onset: 36 y. & & \\
\hline \multirow[t]{2}{*}{ Aiyesimoju [145], 1984} & \multirow[t]{2}{*}{ Nigeria } & \multirow[t]{2}{*}{ Hospital } & \multirow[t]{2}{*}{$\begin{array}{l}\text { Cross sectional } \\
1957-1982\end{array}$} & $\begin{array}{l}2.1 \text { million patients admitted to } \\
\text { the hospital. }\end{array}$ & \multirow[t]{2}{*}{ Not specified } & \multirow[t]{2}{*}{ HD: 0.2/100,000 } \\
\hline & & & & $\begin{array}{l}4 \text { cases (men 3) of HD aged 24-50 y } \\
\text { at diagnosis. }\end{array}$ & & \\
\hline \multirow[t]{2}{*}{ Stephany [146], 1984} & \multirow[t]{2}{*}{ Senegal } & \multirow[t]{2}{*}{ Hospital } & Cross sectional & \multirow{2}{*}{$\begin{array}{l}12370 \text { patients seen in a neurologic clinic; } 3 \\
\text { (men 2) with HD; age } 31-64 \mathrm{y} \text {. }\end{array}$} & Family history & \multirow[t]{2}{*}{$24.2 / 100,000$} \\
\hline & & & 1960-1980 & & $\begin{array}{l}\text { All patients had } \\
\text { movement disorders } \\
\text { and neuropsychiatric } \\
\text { features }\end{array}$ & \\
\hline \multirow[t]{3}{*}{ Joubert [136], 1988} & \multirow[t]{3}{*}{ South Africa } & \multirow[t]{3}{*}{ Community/hospital } & \multirow[t]{3}{*}{$\begin{array}{l}\text { Cross-sectional } \\
\text { 1983-1986 }\end{array}$} & $\begin{array}{l}8 \text { cases in hospital setting }(n=6 \text {. all men) } \\
\text { and at home }(n=2)\end{array}$ & \multirow{3}{*}{$\begin{array}{l}\text { Clinical/genetic } \\
\text { testing/screening } \\
\text { for Wilson disease }\end{array}$} & \multirow[t]{3}{*}{ NA } \\
\hline & & & & Age at onset: 8-47 y. & & \\
\hline & & & & Age at diagnosis: 13-50 y. & & \\
\hline
\end{tabular}


Table 5 Overview of studies on Huntington disease and risk factors in sub-Sahara African countries (Continued)

\begin{tabular}{|c|c|c|c|c|c|c|}
\hline Scrimgeour [152], 1992 & Zimbabwe & Hospital & Case series1991 & $\begin{array}{l}11 \text { cases in a } 4 \text { generation of a single family; } \\
2 \text { probable cases }\end{array}$ & Clinical & $0.5 / 100,000$ \\
\hline \multirow[t]{2}{*}{ Scrimgeour [153], 1995} & \multirow[t]{2}{*}{ Sudan } & \multirow[t]{2}{*}{ Hospital } & \multirow[t]{2}{*}{ Case-report } & 1 case of HD: A & \multirow[t]{2}{*}{ Clinical/MRI } & \multirow[t]{2}{*}{ NA } \\
\hline & & & & 40 year old black Sudanese man & & \\
\hline Grunitzky [154], 1995 & Togo & Hospital & Case series & $\begin{array}{l}\text { A family including } 8 \text { patients with } \mathrm{HD} \text { and } \\
67 \text { at risk across } 6 \text { generations; mean age } \\
\text { at onset: } 33 \mathrm{y} \text {. }\end{array}$ & Not specified & NA \\
\hline Silber [137], 1998 & South Africa & Community & Case series & $\begin{array}{l}5 \text { families of } \mathrm{HD} \text { including a total of } 7 \\
\text { genetically confirmed cases of } \mathrm{HD} \text { and } \\
10 \text { clinically suspect cases of } \mathrm{HD}\end{array}$ & $\begin{array}{l}\text { Clinical/genetic } \\
\text { testing }\end{array}$ & NA \\
\hline Kabore [138], 2000 & Burkina-Faso & Hospital & Case series & 4 cases of HD; age at diagnosis 33-43 y. & $\begin{array}{l}\text { Clinical/genetic } \\
\text { testing }\end{array}$ & $0.04 / 100,000$ \\
\hline \multirow[t]{3}{*}{ Bardien [139], 2007} & \multirow[t]{3}{*}{ South Africa } & \multirow[t]{3}{*}{ Hospital } & \multirow{3}{*}{$\begin{array}{l}\text { Case series } \\
2001-2005\end{array}$} & A family with $\mathrm{HD}$ like 2 & \multirow{3}{*}{$\begin{array}{l}\text { Clinical/genetic } \\
\text { testing }\end{array}$} & \multirow[t]{3}{*}{1} \\
\hline & & & & Total 39 family members & & \\
\hline & & & & 13 had the disease & & \\
\hline Magazi [140], 2008 & South Africa & Hospital & Case series & 12 cases (men 6); age $25-52$ y. & $\begin{array}{l}\text { Clinical/genetic } \\
\text { testing }\end{array}$ & NA \\
\hline
\end{tabular}

HD; Huntington disease; MRI: magnetic resonance imaging; NA: not applicable; NINCDS-ADRDA, National Institute of Neurological and Communicative Disorders and Stroke and the Alzheimer's Disease and Related Disorders Association; y: year. 
factors not properly assessed; thus making comparisons and inferences inaccurate. For these two conditions therefore, important gaps remain to be filled, without which the issues of prevention and control will not be efficiently addressed in the African context.

The comparatively higher number of population-based investigations of dementia relative to other neurodegenerative conditions in SSA, may at least in part be explained by the availability of standardized and widely accepted screening and diagnostic tools/criteria which facilitate epidemiological studies of dementia [157] as compared with other conditions where existing tools have not always been validated in different settings and therefore remain unpopular $[158,159]$, or which, by the virtue of their low prevalence makes any assessment in the general population difficult and very expensive. There are context-specific challenges to obtaining key epidemiological data on neurodegenerative conditions in SSA including the low level of patient education, the need to accurately translate available screening and diagnostic tools to local languages, limited number of scientists and clinicians in neurosciences, and competing health interest in the setting of limited financial resources $[5,16]$.

\section{Needs in terms of epidemiological data}

In order to improve the knowledge base of each of the neurodegenerative conditions addressed in this review, two main types of epidemiological studies appear necessary and feasible in SSA. A population-based prevalence and incidence study including both urban and rural populations, in order to capture the real variability in socioeconomic status and possibility in other factors that may exist in the population. Such a study may serve a dual purpose, providing information on disease rate and identification of key risk factors, as it would permit to establish the sequence of events. Given that such an undertaking could be planned beforehand, it offers the possibility of addressing multiple questions and/or diseases at a reduced cost. Inclusion of a large enough but manageable number of participants would be necessary to ensure adequate precision around the estimates generated. As many patients with possible neurodegenerative conditions would be tempted to consult traditional healers rather than accessing health facilities in SSA, special efforts would be required to ensure that these people are captured by such a study. Also, ascertaining cases of neurodegenerative conditions in a populationbased sample may be costly and logistically challenging, particularly with regard to the asymptomatic or mildly symptomatic nature of early stages of some of the diseases, and the lack of validated instruments and appropriate expertise.

A second type of epidemiological study is a multicenter, hospital-based, registry investigation. The latter has several advantages over a single large-scale cohort study. Large numbers of cases could potentially be collected over a relatively short period of time, with the possibility of comparing resources and outcomes within and across countries. However, the major limitations of this approach include the costs associated with the effort and infrastructure for coordination and communication between centers, as well as data capture and ongoing monitoring and quality control. In addition, there are biases inherent to any such hospital-based study, especially given that in SSA there is major access and cost barriers to care, with a sizeable proportion of patients with neurodegenerative conditions who are never seen by health care providers thus limiting the scope of registries. The degree of such selection bias is likely to vary considerably across centers, affecting both case mix and outcomes. The approach would therefore not provide a study population fully representative of incident cases and the natural history of disease and its management.

For both types of studies, the definition of the pool of people 'at-risk' population could be challenging in the SSA context, given the lack of formal census of the population in many countries; thus making reliable estimation of the effect of individual risk factors difficult. Other methodological issues relate to the assessment of the outcome in a reliable fashion in the African context as discussed above. Hence, a combination of the aforementioned study approaches would probably overcome some of their respective limitations and improve the quality of estimates generated.

The challenges to performing high quality incidence and prevalence studies of neurodegenerative diseases are well known [159]. Cases of most neurodegenerative conditions are difficult to define and ascertain reliably in population-based sample, and there are problems in relating events and the effects of different exposures to defined 'at-risk' populations. With the ageing of the population in SSA, the importance of HIV/AIDS, as well as the surge in risk factors such as hypertension and diabetes that have been linked to dementia $[157,160,161]$ and possibly to Parkinson diseases [162,163], the importance of neurodegenerative disorders would considerably increase over time. Indeed, by 2025 , the numbers of people aged 60 years and over will more than double in many countries [164]. With this rapid demographic and nutritional transition, neurodegenerative conditions would become an important public health problem in SSA. Critical investments are therefore necessary to improve surveillance and programrelevant research to provide an evidence base for policy development and effective control and prevention of neurodegenerative diseases. Precise identification of risk factors other than ageing would allow proper prevention effort spanning from primordial to secondary and event tertiary prevention, given that most of those conditions 
are associated with higher levels of disability and increased risk of death. Community-based risk factor control, combined with high risk approaches and realignment of health systems to incorporate the chronic management of neurodegenerative diseases are needed.

\section{Strengths and limitations of the review}

Our review is the first of its kind on neurodegenerative conditions in SSA. It is more up-to-date and broader than previous attempts to summarize evidence on single diseases in this setting [4-8]. By systematically assessing all published articles on these conditions, we aimed to draw the attention on the importance of the conditions in the region, and identify the research priorities. A limitation of this review is inherent to the limitations of the individual studies included. We relied on clinic-based studies where necessary in this systematic review; but such studies have limitations, particularly with regard to the generalization of their results data. However, we have tried to convey a clear understanding of the current burden and risk factors of each condition by examining all published papers across a broad range of clinical, biology, public health, and psychosocial literature, incorporating various types of evidence. By the nature of the disease, the age range for participants in studies on ALS and HIV-related neurocognitive impairment extended to the pediatric age for some studies. It is of note that large number of studies are realized in hospital in Africa, often published in local journals or reported in thesis. It the absence of straightforward strategies for capturing this sort of evidence in a systematic way, we did not account for them, which may have lowered the number of results found in some countries. Finally, the many sources of heterogeneity precluded any meaningful assessed of the quality of the included studies.

\section{Conclusion}

This review summarizes the body of literature on neurodegenerative disorders in SSA, which is large with regard to Dementia and HIV-related neurocognitive disorders but limited for other neurodegenerative disorders. In addition, it emphasizes some of the challenges in conducting good quality, population-based studies on the continent including the lack of standardized criteria for some neurodegenerative disorders, with most studies limited to few regions/countries on the continent. Highquality prospective cohort studies, which would use internationally- validated criteria, wide catchment areas in several geographic regions, and adjust for the projected ageing of the continent population, by compensating for the imprecise nature of the available data, will help map the epidemiology of neurodegenerative diseases in SSA and improve comparisons with the rest of the world.

\section{Additional file}

Additional file 1: Search terms and strategies.

Competing interest

The authors declare that they have no competing interests.

Authors' contribution

All authors equally contributed. All authors read and approved the final manuscript.

\section{Author details}

${ }^{1}$ Department of Neurosciences, Division of Neurology, Medical University of South Carolina, Charleston, USA. ${ }^{2}$ Hubert Department of Global Health, Rollins School of Public Health, Emory University, Atlanta, Georgia, USA. ${ }^{3}$ MedStar Health, Baltimore, Maryland, USA. ${ }^{4}$ Department of Medicine, University of Cape Town, Cape Town, South Africa. ${ }^{5}$ The George Institute for Global Health, Sydney, Australia. ${ }^{6} J u l i u s$ Center for Health Sciences and Primary Care, University Medical Center Utrecht, Utrecht, the Netherlands. ${ }^{7}$ Non-Communicable Diseases Research Unit, South African Medical Research Council, PO Box 19070 Tygerberg, Cape Town 7505, South Africa.

Received: 9 September 2013 Accepted: 19 May 2014

Published: 26 June 2014

\section{References}

1. World Population Prospects: The 2012 Revision. http://esa.un.org/unpd/ wpp/unpp/panel_indicators.htm.

2. World Population Ageing 2013. http://www.un.org/en/development/desa/ population/publications/pdf/ageing/WorldPopulationAgeingReport2013.pdf.

3. Mateen FJ, Mills EJ: Aging and HIV-related cognitive loss. Jama 2012, 308(4):349-350.

4. Okubadejo NU, Bower JH, Rocca WA, Maraganore DM: Parkinson's disease in Africa: A systematic review of epidemiologic and genetic studies. Mov Disord 2006, 21(12):2150-2156.

5. Cilia R, Akpalu A, Cham M, Bonetti A, Amboni M, Faceli E, Pezzoli G: Parkinson's disease in sub-Saharan Africa: step-by-step into the challenge. Neurodegener Dis Manag 2011, 1(3):194-202.

6. Ineichen B: The epidemiology of dementia in Africa: a review. Soc Sci Med 2000, 50(11):1673-1677.

7. George-Carey R, Adeloye D, Chan KY, Paul A, Kolcic I, Campbell H, Rudan I: An estimate of the prevalence of dementia in Africa: A systematic analysis. J Glob Health 2012, 2(2):20401.

8. Marin B, Kacem I, Diagana M, Boulesteix M, Gouider R, Preux PM, Couratier P: Juvenile and adult-onset ALS/MND among Africans: incidence, phenotype, survival: a review. Amyotroph Lateral Scler 2012, 13(3):276-283.

9. Hendrie HC, Murrell J, Gao S, Unverzagt FW, Ogunniyi A, Hall KS: International studies in dementia with particular emphasis on populations of African origin. Alzheimer Dis Assoc Disord 2000, 20:S42-S46.

10. Bower JH, Teshome M, Melaku Z, Zenebe G: Frequency of movement disorders in an Ethiopian university practice. Mov Disord 2005, 20(9):1209-1213.

11. Akinyemi RO, Okubadejo NN, Akinyemi JO, Owolabi MO, Owolabi LF, Ogunniyi A: Cognitive dysfunction in Nigerians with Parkinson's disease. Mov Disord 2008, 23(10):1378-1383.

12. Cosnett JE, Bill PL: Parkinson's disease in blacks. Observations on epidemiology in Natal. S Afr Med J 1988, 73(5):281-283.

13. Dotchin C, Msuya O, Kissima J, Massawe J, Mhina A, Moshy A, Aris E, Jusabani A, Whiting D, Masuki G, Walker R: The prevalence of Parkinson's disease in rural Tanzania. Mov Disord 2008, 23(11):1567-1672.

14. Schoenberg BS, Osuntokun BO, Adeuja AO, Bademosi O, Nottidge V, Anderson DW, Haerer AF: Comparison of the prevalence of Parkinson's disease in black populations in the rural United States and in rural Nigeria: door-to-door community studies. Neurology 1988, 38(4):645-646.

15. Winkler AS, Tutuncu E, Trendafilova A, Meindl M, Kaaya J, Schmutzhard E, Kassubek J: Parkinsonism in a population of northern Tanzania: a community-based door-to-door study in combination with a prospective hospital-based evaluation. J Neurol 2010, 257(5):799-805. 
16. Kengne AP, Dzudie A, Dongmo L: Epidemiological features of degenerative brain diseases as they occurred in Yaounde referral hospitals over a 9-year period. Neuroepidemiology 2006, 27(4):208-211.

17. Lombard A, Gelfand M: Parkinson's disease in the African. Cent Afr J Med 1978, 24(1):5-8.

18. Osuntokun $\mathrm{BO}$, Bademosi O: Parkinsonism in the Nigerian African: a prospective study of 217 patients. East Afr Med J 1979, 56(11):597-607.

19. Osuntokun BO, Adeuja AO, Schoenberg BS, Bademosi O, Nottidge VA, Olumide AO, Ige O, Yaria F, Bolis CL: Neurological disorders in Nigerian Africans: a community-based study. Acta Neurol Scand 1987, 75(1):13-21.

20. Haylett WL, Keyser RJ, du Plessis MC, van der Merwe C, Blanckenberg J, Lombard D, Carr J, Bardien S: Mutations in the parkin gene are a minor cause of Parkinson's disease in the South African population. Parkinsonism Relat Disord 2012, 18(1):89-92.

21. Ekenze OS, Onwuekwe IO, Ezeala Adikaibe BA: Profile of neurological admissions at the University of Nigeria Teaching Hospital Enugu. Niger Med 2010, 19(4):419-422.

22. Owolabi LF, Shehu MY, Shehu MN, Fadare J: Pattern of neurological admissions in the tropics: Experience at Kano, Northwestern Nigeria. Ann Indian Acad Neurol 2010, 13(3):167-170.

23. Okubadejo NU, Ojini Fl, Danesi MA: Longitudinal study of mortality predictors in Parkinson's disease in Nigerians. Afr J Med Med Sci 2005, 34(4):365-369.

24. Okubadejo NU, Danesi MA: Frequency and predictors of autonomic dysfunction in Parkinson's disease: a study of African patients in Lagos, Nigeria. Niger Postgrad Med J 2004, 11(1):45-49.

25. Okubadejo NU, Ojo OO, Oshinaike OO: Clinical profile of parkinsonism and Parkinson's disease in Lagos, Southwestern Nigeria. BMC neurology 2010, 10:1

26. Keyser RJ, Lesage S, Brice A, Carr J, Bardien S: Assessing the prevalence of PINK1 genetic variants in South African patients diagnosed with earlyand late-onset Parkinson's disease. Biochem Biophys Res Commun 2010, 398(1):125-129.

27. van der Merwe C, Haylett W, Harvey J, Lombard D, Bardien S, Carr J: Factors influencing the development of early- or late-onset Parkinson's disease in a cohort of South African patients. S Afr Med J 2012, 102(11 Pt 1):848-851.

28. Femi OL, Ibrahim A, Aliyu S: Clinical profile of parkinsonian disorders in the tropics: Experience at Kano, northwestern Nigeria. J Neurosci Rural Pract 2012, 3(3):237-241.

29. Cilia R, Sironi F, Akpalu A, Cham M, Sarfo FS, Brambilla T, Bonetti A, Ambon M, Goldwurm S, Pezzoli G: Screening LRRK2 gene mutations in patients with Parkinson's disease in Ghana. J Neurol 2012, 259(3):569-570.

30. Amoo G, Akinyemi RO, Onofa LU, Akinyemi JO, Baiyewu O, Ogunlesi AO, Ogunniyi A: Profile of clinically-diagnosed dementias in a neuropsychiatric practice in Abeokuta, South-Western Nigeria. Afr J Psychiatry 2011, 14(5):377-382

31. Ndiaye D, Sylla A, Toure K, Thiam MH, Gueye M: Bilan de fonctionnement d'une clinique de la memoire senegalaise. AJNS 2011, 30(1):1-9.

32. Hendrie HC, Osuntokun BO, Hall KS, Ogunniyi AO, Hui SL, Unverzagt FW Gureje O, Rodenberg CA, Baiyewu O, Musick BS: Prevalence of Alzheimer's disease and dementia in two communities: Nigerian Africans and African Americans. Am J Psychiatry 1995, 152(10):1485-1492.

33. Ogeng'O JA, Cohen DL, Sayi JG, Matuja WB, Chande HM, Kitinya JN, Kimani JK, Friedland RP, Mori H, Kalaria RN: Cerebral amyloid beta protein deposits and other Alzheimer lesions in non-demented elderly east Africans. Brain pathology 1996, 6(2):101-107.

34. Hall K, Gureje O, Gao S, Ogunniyi A, Hui SL, Baiyewu O, Unverzagt FW, Oluwole S, Hendrie HC: Risk factors and Alzheimer's disease: a comparative study of two communities. Aust N Z J Psychiatry 1998, 32(5):698-706.

35. Hendrie HC, Ogunniyi A, Hall KS, Baiyewu O, Unverzagt FW, Gureje O, Gao S, Evans RM, Ogunseyinde AO, Adeyinka AO, Musick B, Hui SL: Incidence of dementia and Alzheimer disease in 2 communities: Yoruba residing in Ibadan, Nigeria, and African Americans residing in Indianapolis, Indiana. JAMA 2001, 285(6):739-747.

36. Perkins AJ, Hui SL, Ogunniyi A, Gureje O, Baiyewu O, Unverzagt FW, Gao S, Hall KS, Musick BS, Hendrie HC: Risk of mortality for dementia in a developing country: the Yoruba in Nigeria. Int J Geriatr Psychiatry 2002, 17(6):566-573.

37. Lane KA, Gao S, Hui SL, Murrell JR, Hall KS, Hendrie HC: Apolipoprotein E and mortality in African-Americans and Yoruba. J Alzheimers Dis 2003 5(5):383-390.
38. Osuntokun BOOA, Junaid TA, Lekwauwa UG: Autopsy survey for Alzheimer's disease in Nigerian Africans: a preliminary report. Afr J Med Med Sci 1995, 24(1):75-79.

39. Ben-Arie OSL, Teggin AF, Elk R: The coloured elderly in Cape Town-a psychosocial, psychiatric and medical community survey. Part II. Prevalence of psychiatric disorders. S Afr Med J 1983, 64(27):1056-1061.

40. Ogunniyi AO, Osuntokun BO, Lekwauwa UB, Falope ZF: Rarity of dementia (by DSM-III-R) in an urban community in Nigeria. East Afr Med J 1992, 69(2):64-68

41. Osuntokun BO, Sahota A, Ogunniyi AO, Gureje O, Baiyewu O, Adeyinka A, Oluwole SO, Komolafe O, Hall KS, Unverzagt FW, Hui SL, Yang M, Hendrie HC: Lack of an association between apolipoprotein E epsilon 4 and Alzheimer's disease in elderly Nigerians. Ann Neurol 1995, 38(3):463-465.

42. Ogunniyi A, Gureje O, Baiyewu O, Unverzagt F, Hall KS, Oluwole S, Osuntokun BO, Hendrie HC: Profile of dementia in a Nigerian community-types, pattern of impairment, and severity rating. $J$ Natl Med Assoc 1997, 89(6):392-396.

43. Ogunniyi A, Baiyewu O, Gureje O, Hall KS, Unverzagt F, Siu SH, Gao S, Farlow M, Oluwole OS, Komolafe O, Hendrie HC: Epidemiology of dementia in Nigeria: results from the Indianapolis-lbadan study. Eur $J$ Neurol 2000, 7(5):485-490.

44. Baiyewu O, Unverzagt FW, Ogunniyi A, Hall KS, Gureje O, Gao S, Lane KA, Hendrie $\mathrm{HC}$ : Cognitive impairment in community-dwelling older Nigerians: clinical correlates and stability of diagnosis. Eur J Neurol 2002, 9(6):573-580.

45. Ogunniyi A, Hall KS, Baiyewu O, Gureje O, Unverzagt FW, Gao S, Hendrie HC Caring for individuals with dementia: the Nigerian experience. West Afr J Med 2005, 24(3):259-262

46. Gureje O, Ogunniyi A, Kola L: The profile and impact of probable dementia in a sub-Saharan African community: Results from the Ibadan Study of Aging. J Psychosom Res 2006, 61(3):327-333.

47. Ochayi B, Thacher TD: Risk factors for dementia in central Nigeria. Aging Ment Health 2006, 10(6):616-620.

48. Hall K, Murrell J, Ogunniyi A, Deeg M, Baiyewu O, Gao S, Gureje O, Dickens J, Evans R, Smith-Gamble V, Unverzagt FW, Shen J, Hendrie H: Cholesterol, APOE genotype, and Alzheimer disease: an epidemiologic study of Nigerian Yoruba. Neurology 2006, 66(2):223-227.

49. Guerchet M, M'Belesso P, Mouanga AM, Bandzouzi B, Tabo A, Houinato DS, Paraiso MN, Cowppli-Bony P, Nubukpo P, Aboyans V, Clément JP, Dartigues $J$, Preux PM: Prevalence of dementia in elderly living in two cities of Central Africa: the EDAC survey. Dement Geriatr Cogn Disord 2010, 30(3):261-268

50. Guerchet M, Houinato D, Paraiso MN, von Ahsen N, Nubukpo P, Otto M, Clement JP, Preux PM, Dartigues JF: Cognitive impairment and dementia in elderly people living in rural Benin, west Africa. Dement Geriatr Cogn Disord 2009, 27(1):34-41.

51. Gureje O, Ogunniyi A, Kola L, Abiona T: Incidence of and risk factors for dementia in the lbadan study of aging. J Am Geriatr Soc 2011, 59(5):869-874.

52. Ogunniyi A, Gao S, Unverzagt FW, Baiyewu O, Gureje O, Nguyen JT, Smith-Gamble V, Murrell JR, Hake AM, Hall KS, Hendrie HC: Weight loss and incident dementia in elderly Yoruba Nigerians: a 10-year follow-up study. Int Psychogeriatr 2011, 23(3):387-394.

53. Ogunniyi A, Lane KA, Baiyewu O, Gao S, Gureje O, Unverzagt FW, Murrell JR, Smith-Gamble V, Hall KS, Hendrie HC: Hypertension and incident dementia in community-dwelling elderly Yoruba Nigerians. Acta Neurol Scand 2011, 124(6):396-402.

54. Baiyewu O, Unverzagt FW, Ogunniyi A, Smith-Gamble V, Gureje O, Lane KA, Gao S, Hall KS, Hendrie HC: Behavioral symptoms in community-dwelling elderly Nigerians with dementia, mild cognitive impairment, and normal cognition. Int J Geriatr Psychiatry 2012, 27(9):931-939.

55. Guerchet M, Mouanga AM, M'Belesso P, Tabo A, Bandzouzi B, Paraiso MN, Houinato DS, Cowppli-Bony P, Nubukpo P, Aboyans V, Clément JP Dartigues JF, Preux PM: Factors associated with dementia among elderly people living in two cities in Central Africa: the EDAC multicenter study. J Alzheimers Dis 2012, 29(1):15-24.

56. Paraiso MN, Guerchet M, Saizonou J, Cowppli-Bony P, Mouanga AM, Nubukpo P, Preux PM, Houinato DS: Prevalence of Dementia among Elderly People Living in Cotonou, an Urban Area of Benin (West Africa). Neuroepidemiology 2011, 36(4):245-251.

57. Longdon AR, Paddick SM, Kisoli A, Dotchin C, Gray WK, Dewhurst F, Chaote P, Teodorczuk A, Dewhurst M, Jusabani AM, Walker R: The prevalence of 
dementia in rural Tanzania: a cross-sectional community-based study. Int J Geriatr Psychiatry 2013, 28(7):728-737.

58. Lambo TA: Psychiatric disorders in the aged: epidemiology and preventive measures. West Afr Med J 1966, 15(3):121-124.

59. Makanjuola RO: Psychiatric disorders in elderly Nigerians. Trop Geogr Med 1985, 37(4):348-351.

60. Gureje O, Osuntokun BO, Makanjuola JD: Neuropsychiatric disorders in Nigerians: 1914 consecutive new patients seen in 1 year. Afr J Med Med Sci 1989, 18(3):203-209.

61. Osuntokun BO, Ogunniyi A, Akang EE, Aghadiuno PU, llori A, Bamgboye EA, Beyreuther K, Masters C: Beta A4-amyloid in the brains of non-demented Nigerian Africans. Lancet 1994, 343(8888):56.

62. Sayi JG, Patel NB, Premkumar DR, Adem A, Winblad B, Matuja WB, Mtui EP, Gatere S, Friedland RP, Koss E, Kalaria RN: Apolipoprotein E polymorphism in elderly east Africans. East Afr Med J 1997, 74(10):668-670.

63. Baiyewu O, Adeyemi JD, Ogunniyi A: Psychiatric disorders in Nigerian nursing home residents. Int J Geriatr Psychiatry 1997, 12(12):1146-1150.

64. Uwakwe R: Satisfaction with dementia care-giving in Nigeria-a pilot investigation. Int J Geriatr Psychiatry 2006, 21(3):296-297.

65. Chen CH, Mizuno T, Elston R, Kariuki MM, Hall K, Unverzagt F, Hendrie H, Gatere S, Kioy P, Patel NB, Friedland RP, Kalaria RN: A comparative study to screen dementia and APOE genotypes in an ageing East African population. Neurobiol Aging 2010, 31(5):732-740.

66. Toure KCM, Ndiaye M, Zunzunegui MV, Bacher Y, Diop AG, Ndiaye MM: Risk factors for dementia in a senegalese elderly population aged 65 years and over. Dement Geriatr Cogn Dis 2012, 2:160-168.

67. Toure K, Coume M, Ndiaye ND, Thiam MH, Zunzunegui MV, Bacher Y, Tal DA, Gueye L, Sene-Diouf F, Ndiaye M, Thiam A, Amadou GD, Ndiaye MM: Facteur de risque de démence dans une population de personne âgées sénégalaises. Afr J Neurol Sci 2009, 28(1):1-15.

68. Napon CTI, Niakara A, Ouango JG, Kabre A, Kabore J: Les demences en Afrique sub-Saharienne : Aspects cliniques et etiologiques en milieu hospitalier a Ouagadougou (Bourkina Fasso). AJNS 2009, 28(1):37-43.

69. Siddiqi OK, Atadzhanov M, Birbeck GL, Koralnik IJ: The spectrum of neurological disorders in a Zambian tertiary care hospital. J Neurol Sci 2009, 290(1-2):1-5.

70. Uwakwe R: Psychiatric morbidity in elderly patients admitted to nonpsychiatric wards in a general/teaching hospital in Nigeria. Int I Geriatr Psychiatry 2000, 15(4):346-354.

71. Gureje O, Ogunniyi A, Baiyewu O, Price B, Unverzagt FW, Evans RM, SmithGamble V, Lane KA, Gao S, Hall KS, Hendrie HC, Murrell JR: APOE epsilon4 is not associated with Alzheimer's disease in elderly Nigerians. Ann Neurol 2006, 59(1):182-185

72. Ogunniyi A, Hall KS, Gureje O, Baiyewu O, Gao S, Unverzagt FW, SmithGamble V, Evans RE, Dickens J, Musick BS, Hendrie C: Risk factors for incident Alzheimer's disease in African Americans and Yoruba. Metab Brain Dis 2006, 21(2-3):235-240.

73. Uwakwe R, Ibeh CC, Modebe Al, Bo E, Ezeama N, Njelita I, Ferri CP, Prince MJ: The epidemiology of dependence in older people in Nigeria: prevalence, determinants, informal care, and health service utilization. A 10/66 dementia research group cross-sectional survey. J Am Geriatr SoC 2009, 57(9):1620-1627.

74. Yusuf AJ, Baiyewu O, Sheikh TL, Shehu AU: Prevalence of dementia and dementia subtypes among community-dwelling elderly people in northern Nigeria. Int Psychogeriatr 2011, 23(3):379-386.

75. Coume M, Toure K, Thiam MH, Zunzunegui MV, Bacher Y, Diop TM, Ndiaye MM: Estimate of the prevalence of cognitive impairment in an elderly population of the health center of Senegalese national retirement institution. Geriatr Psychol Neuropsychiatr Vieil 2012, 10(1):39-46.

76. Onwuekwe I: Assessment of mild cognitive impairment with mini mental state examination among adults in southeast Nigeria. Ann Med Health Sci Res 2012, 2(2):99-102.

77. Belec L, Testa J, Vohito MD, Gresenguet G, Martin MI, Tabo A, Di Costanzo B, Georges AJ: Neurologic and psychiatric manifestations of AIDS in Central African Republic. Bull Soc Pathol Exot Filiales 1989, 82(3):297-307.

78. Howlett WP, Nkya WM, Mmuni KA, Missalek WR: Neurological disorders in AIDS and HIV disease in the northern zone of Tanzania. AIDS 1989, 3(5):289-296.

79. Turnbull O, Saling MM, Kaplan-Solms K, Cohn R, Schoub B: Neuropsychological deficit in haemophiliacs with human immunodeficiency virus. J Neurol Neurosurg Psychiatry 1991, 54(2):175-177.
80. Perriens JH, Mussa M, Luabeya MK, Kayembe K, Kapita B, Brown C, Piot P, Janssen R: Neurological complications of HIV-1-seropositive internal medicine inpatients in Kinshasa, Zaire. J Acquir Immune Defic Syndr 1992, 5(4):333-340.

81. Maj M, Satz P, Janssen R, Zaudig M, Starace F, D'Elia L, Sughondhabirom B, Mussa M, Naber D, Ndetei D, Schulte G, Sartorius N: WHO Neuropsychiatric AIDS study, cross-sectional phase II. Neuropsychological and neurological findings. Arch Gen Psychiatry 1994, 51(1):51-61.

82. Carson AJ, Sandler R, Owino FN, Matete FO, Johnstone EC: Psychological morbidity and HIV in Kenya. Acta Psychiatr Scand 1998, 97(4):267-271.

83. Sebit MB: Neuropsychiatric HIV-1 infection study: in Kenya and Zaire cross-sectional phase I and II. Cent Afr J Med 1995, 41(10):315-322.

84. Sacktor N, Nakasujja N, Skolasky R, Robertson K, Wong M, Musisi S, Ronald A, Katabira E: Antiretroviral therapy improves cognitive impairment in HIV + individuals in sub-Saharan Africa. Neurology 2006, 67(2):311-314.

85. Sacktor NC, Wong M, Nakasujja N, Skolasky RL, Selnes OA, Musisi S, Robertson K, McArthur JC, Ronald A, Katabira E: The International HIV Dementia Scale: a new rapid screening test for HIV dementia. AIDS 2005, 19(13):1367-1374.

86. Modi G, Hari K, Modi M, Mochan A: The frequency and profile of neurology in black South African HIV infected (clade C) patients - a hospital-based prospective audit. J Neurol Sci 2007, 254(1-2):60-64.

87. Clifford DB, Mitike MT, Mekonnen Y, Zhang J, Zenebe G, Melaku Z, Zewde A, Gessesse N, Wolday D, Messele T, Teshome M, Evans S: Neurological evaluation of untreated human immunodeficiency virus infected adults in Ethiopia. J Neurovirol 2007, 13(1):67-72.

88. Odiase FE, Ogunrin OA, Ogunniyi AA: Memory performance in HIV/AIDS-a prospective case control study. Can J Neurol Sci 2007, 34(2):154-159.

89. Wong MH, Robertson K, Nakasujja N, Skolasky R, Musisi S, Katabira E, McArthur JC, Ronald A, Sacktor N: Frequency of and risk factors for HIV dementia in an HIV clinic in sub-Saharan Africa. Neurology 2007, 68(5):350-355.

90. Robertson KR, Nakasujja N, Wong M, Musisi S, Katabira E, Parsons TD, Ronald A, Sacktor N: Pattern of neuropsychological performance among HIV positive patients in Uganda. BMC Neurol 2007, 7:8.

91. Salawu FK, Bwala SA, Wakil MA, Bani B, Bukbuk DN, Kida I: Cognitive function in HIV-seropositive Nigerians without AIDS. J Neurol Sci 2008, 267(1-2):142-146.

92. Singh D, Sunpath $H$, John S, Eastham L, Gouden R: The utility of a rapid screening tool for depression and HIV dementia amongst patients with low CD4 counts- a preliminary report. Afr J Psychiatry 2008, 11(4):282-286.

93. Sall L, Salamon E, Allgulander C, Owe-Larsson B: Psychiatric symptoms and disorders in HIV infected mine workers in South Africa. A retrospective descriptive study of acute first admissions. Afr J Psychiatry 2009, 12(3):206-212.

94. Ganasen KA, Fincham D, Smit J, Seedat S, Stein D: Utility of the HIV Dementia Scale (HDS) in identifying HIV dementia in a South African sample. J Neurol Sci 2008, 269(1-2):62-64.

95. Njamnshi AKDVP, Fonsah JY, Yepnjio FN, Njamnshi DM, Muna WE: The International HIV Dementia Scale is a useful screening tool for HIVassociated dementia/cognitive impairment in HIV-infected adults in Yaoundé-Cameroon. J Acquir Immune Defic Syndr 2008, 49(4):393-397.

96. Sacktor N, Nakasujja N, Skolasky RL, Robertson K, Musisi S, Ronald A, Katabira E, Clifford DB: Benefits and risks of stavudine therapy for HIV-associated neurologic complications in Uganda. Neurology 2009, 72(2):165-170.

97. Njamnshi AKBA, Ongolo-Zogo P, Tabah EN, Lekoubou AZ, Yepnjio FN, Fonsah JY, Kuate CT, Angwafor SA, Dema F, Njamnshi DM, Kouanfack C, Djientcheu Vde P, Muna WF, Kanmogne GD: Risk factors for HIV-associated neurocognitive disorders (HAND) in sub-Saharan Africa: The case of Yaoundé-Cameroon. J Neurol Sci 2009, 285(1-2):149-153.

98. Sacktor N, Nakasujja N, Skolasky RL, Rezapour M, Robertson K, Musisi S, Katabira E, Ronald A, Clifford DB, Laeyendecker O, Quinn TC: HIV subtype D is associated with dementia, compared with subtype $A$, in immunosuppressed individuals at risk of cognitive impairment in Kampala, Uganda. Clin Infec Dis 2009, 49(5):780-786.

99. Nakasujja N, Skolasky RL, Musisi S, Allebeck P, Robertson K, Ronald A, Katabira E, Clifford DB, Sacktor N: Depression symptoms and cognitive function among individuals with advanced HIV infection initiating HAART in Uganda. BMC Psychiatry 2010, 10:44.

100. Kinyanda E, Hoskins S, Nakku J, Nawaz S, Patel V: Prevalence and risk factors of major depressive disorder in HIV/AIDS as seen in semi-urban Entebbe district, Uganda. BMC Psychiatry 2011, 11:205. 
101. Choi Y, Townend J, Vincent T, Zaidi I, Sarge-Njie R, Jaye A, Clifford DB: Neurologic manifestations of human immunodeficiency virus-2: dementia, myelopathy, and neuropathy in West Africa. J Neurovirol 2011, 17(2):166-175.

102. Birbeck GL, Kvalsund MP, Byers PA, Bradbury R, Mang'ombe C, Organek N, Kaile T, Sinyama AM, Sinyangwe SS, Malama K, Malama C: Neuropsychiatric and socioeconomic status impact antiretroviral adherence and mortality in rural Zambia. Am J Trop Med Hyg 2011, 85(4):782-789.

103. Joska JA, Fincham DS, Stein DJ, Paul RH, Seedat S: Clinical correlates of HIV-associated neurocognitive disorders in South Africa. AIDS Behav 2010, 14(2):371-378

104. Kanmogne GD, Kuate CT, Cysique LA, Fonsah JY, Eta S, Doh R, Njamnshi DM, Nchindap E, Franklin DR Jr, Ellis RJ, McCutchan JA, Binam F, Mbanya D, Heaton RK, Njamnshi AK: HIV-associated neurocognitive disorders in subSaharan Africa: a pilot study in Cameroon. BMC neurology 2010, 10:60.

105. Lawler K, Mosepele M, Ratcliffe S, Seloilwe E, Steele K, Nthobatsang R, Steenhoff A: Neurocognitive impairment among HIV-positive individuals in Botswana: a pilot study. J Int AIDS Soc 2010, 13:15.

106. Patel VN, Mungwira RG, Tarumbiswa TF, Heikinheimo T, van Oosterhout J: High prevalence of suspected HIV-associated dementia in adult Malawian HIV patients. Int J STD AIDS 2010, 21(5):356-358.

107. Holguin A, Banda M, Willen EJ, Malama C, Chiyenu KO, Mudenda VC, Wood C: HIV-1 effects on neuropsychological performance in a resourcelimited country, Zambia. AIDS and behavior 2011, 15(8):1895-1901.

108. Joska JA, Westgarth-Taylor J, Hoare J, Thomas KG, Paul R, Myer L, Stein DJ: Validity of the International HIV Dementia Scale in South Africa. AIDS Patient Care STDS 2011, 25(2):95-101.

109. Obiabo YO, Ogunrin OA, Ogun AS: Effects of highly active antiretroviral therapy on cognitive functions in severely immune-compromised HIVseropositive patients. J Neurol Sci 2012, 313(1-2):115-122.

110. Joska JA, Westgarth-Taylor J, Myer L, Hoare J, Thomas KG, Combrinck M, Paul RH, Stein DJ, Flisher AJ: Characterization of HIV-Associated Neurocognitive Disorders among individuals starting antiretroviral therapy in South Africa. AIDS Behav 2011, 15(6):1197-1203.

111. Robertson K, Kumwenda J, Supparatpinyo K, Jiang JH, Evans S, Campbell TB, Price RW, Murphy R, Hall C, Marra CM, Marcus C, Berzins B, Masih R, Santos B, Silva MT, Kumarasamy N, Walawander A, Nair A, Tripathy S, Kanyama C, Hosseinipour M, Montano S, La Rosa A, Amod F, Sanne I, Firnhaber C, Hakim J, Brouwers P, AIDS Clinical Trials Group: A multinational study of neurological performance in antiretroviral therapy-naive HIV-1-infected persons in diverse resource-constrained settings. J Neurovirol 2011, 17(5):438-447.

112. Robbins RN, Remien RH, Mellins CA, Joska JA, Stein DJ: Screening for HIVassociated dementia in South Africa: potentials and pitfalls of taskshifting. AIDS patient care and STDs 2011, 25(10):587-593.

113. Kwasa J, Cettomai D, Lwanya E, Osiemo D, Oyaro P, Birbeck GL, Price RW Bukusi EA, Cohen CR, Meyer AC: Lessons learned developing a diagnostic tool for HIV-associated dementia feasible to implement in resourcelimited settings: pilot testing in Kenya. PLoS One 2012, 7(3):e32898

114. Spies G, Fennema-Notestine C, Archibald SL, Cherner M, Seedat S: Neurocognitive deficits in HIV-infected women and victims of childhood trauma. AIDS Care 2012, 24(9):1126-1135.

115. Hestad KA, Menon JA, Silalukey-Ngoma M, Franklin DR Jr, Imasiku ML, Kalima K, Heaton RK: Sex differences in neuropsychological performance as an effect of human immunodeficiency virus infection: a pilot study in Zambia. Africa. J Nerv Ment Dis 2012, 200(4):336-342.

116. Berhe T, Melkamu Y, Amare A: The pattern and predictors of mortality of HIV/AIDS patients with neurologic manifestation in Ethiopia: a retrospective study. AIDS Res Ther 2012, 9:11.

117. Joska JA, Westgarth-Taylor J, Hoare J, Thomas KG, Paul R, Myer L, Stein DJ: Neuropsychological outcomes in adults commencing highly active antiretroviral treatment in South Africa: a prospective study. BMC infectious diseases 2012, 12:39.

118. Breuer E, Stoloff K, Myer L, Seedat S, Stein DJ, Joska J: Reliability of the Lay Adherence Counsellor Administered Substance Abuse and Mental Illness Symptoms Screener (SAMISS) and the International HIV Dementia Scale (IHDS) in a Primary care HIV Clinic in Cape Town, South Africa. AIDS and behavior 2012, 16(6):1464-1471.

119. Hoare J, Westgarth-Taylor J, Fouche JP, Combrinck M, Spottiswoode B, Stein DJ, Joska JA: Relationship between apolipoprotein E4 genotype and white matter integrity in HIV-positive young adults in South Africa. Eur Arch Psychiatry Clin Neurosci 2013, 263(3):189-195.
120. Oshinaike OO, Akinbami AA, Ojo OO, Ojini IF, Okubadejo UN, Danesi AM: Comparison of the Minimental State Examination Scale and the International HIV Dementia Scale in Assessing Cognitive Function in Nigerian HIV Patients on Antiretroviral Therapy. AIDS Res Treat 2012, 2012:581531.

121. Royal W 3rd, Cherner M, Carr J, Habib AG, Akomolafe A, Abimiku A, Charurat M, Farley J, Oluyemisi A, Mamadu I, Johnson J, Ellis R, McCutchan JA, Grant I, Blattner WA: Clinical features and preliminary studies of virological correlates of neurocognitive impairment among HIV-infected individuals in Nigeria. J Neurovirol 2012, 18(3):191-199.

122. Tekle-Haimanot R, Abebe M, Gebre-Mariam A, Forsgren L, Heijbel J, Holmgren $\mathrm{G}$, Ekstedt J: Community-based study of neurological disorders in rural central Ethiopia. Neuroepidemiology 1990, 9(5):263-277.

123. Jacquin-Cotton L, Dumas M, Girard PL: Paraplegia in Senegal. Bull Soc Med Afr Noire Lang Fr 1970, 15(2):206-220.

124. Piquemal M, Beugre K, Boa Y, Giordano C: Etude de 30 observations de Syndrome de sclérose latérale Amyotrophique observés en Côte d'Ivoire. Afr J Neurol Sci 1982, 1:31-40

125. Cosnett JE, Bill PL, Bhigjee Al: Motor neuron disease in blacks. Epidemiological observations in Natal. S Afr Med J 1989, 76(4):155-157.

126. Osuntokun $\mathrm{BO}$, Adeuja $\mathrm{AO}$, Bademosi O: The prognosis of motor neuron disease in Nigerian africans. A prospective study of 92 patients. $J$ Neurol 1974, 97(2):385-394.

127. Abdulla MN, Sokrab TE, el Tahir A, Siddig HE, Ali ME: Motor neurone disease in the tropics: findings from Sudan. East Afr Med J 1997, 74(1):46-48.

128. Sene DF, Ndiaye $M$, Toure $K$, Ndao AK, Thiam A, Diop AG, Ndiaye IP: Epidemiological and clinical aspects of amyotrophic lateral sclerosis in neurological clinic of Dakar. Dakar Med 2004, 49(3):167-171.

129. Adam AM: Unusual form of motor neuron disease in Kenya. East Afr Med J 1992, 69(2):55-57.

130. Wall DW, Gelfand M: Motor neuron disease in Rhodesian Africans. J Neurol 1972, 95(3):517-520

131. Imam I, Ogunniyi A: What is happening to motor neuron diseases in Nigeria? Ann African Medicine 2004, 3(1):1-3

132. Harries JR: Amyotrophic lateral sclerosis in Africans. East Afr Med J 1955, 32(8):333-335

133. Collomb H, Virieu R, Dumas M, Lemercier G: Maladie de Charcot et syndromes de sclérose latérale amyotrophique au sénégal: Etude clinique de 27 observations 1968; 13(4):785-804. Bull Soc Med Noire Lque Fr 1968, 13(4):785-804

134. Mutesa L, Pierquin G, Segers K, Vanbellinghen JF, Gahimbare L, Bours V: Spinocerebellar ataxia type 2 (SCA2): clinical features and genetic analysis. J Trop Pediatr 2008, 54(5):350-352.

135. Traore H, Diagana M, Prieux PM, Debrock C, Ba A, Algadi B,MD: Pathologie hérédodégénérative du système nerveux central dans un service de neurologie à Nouakchott. Afr J Neurol Sci 1998, 17:30-36.

136. Joubert J, Botha MC: Huntington disease in South African blacks. A report of 8 cases. S Afr Med J 1988, 73(8):489-494.

137. Silber E, Kromberg J, Temlett JA, Krause A, Saffer D: Huntington's disease confirmed by genetic testing in five African families. Mov Disord 1998, 13(4):726-730

138. Kabore J, Ouedraogo A: Huntington disease in Burkina Faso. Rev Neurol (Paris) 2000, 156(12):1157-1158

139. Bardien S, Abrahams F, Soodyall H, van der Merwe L, Greenberg J, Brink T, Carr J: A South African mixed ancestry family with Huntington diseaselike 2: clinical and genetic features. Mov Disord 2007, 22(14):2083-2089.

140. Magazi DS, Krause A, Bonev V, Moagi M, Iqbal Z, Dludla M, van der Meyden $\mathrm{CH}$ : Huntington's disease: genetic heterogeneity in black African patients. S Afr Med J 2008, 98(3):200-203.

141. Hayden MR, Beighton P: Huntington's chorea in the Cape coloured community of South Africa. S Afr Med J 1977, 52(22):886-888.

142. Hayden MR, MacGregor JM, Beighton PH: The prevalence of Huntington's chorea in South Africa. S Afr Med J 1980, 58(5):193-196.

143. Hayden MR, Berkowicz AL, Beighton PH, Yiptong C: Huntington's chorea on the island of Mauritius. S Afr Med J 1981, 60(26):1001-1002.

144. Hayden MR, MacGregor JM, Saffer DS, Beighton PH: The high frequency of juvenile Huntington's chorea in South Africa. J Med Genet 1982, 19(2):94-97.

145. Aiyesimoju AB, Osuntokun BO, Bademosi O, Adeuja AO: Hereditary neurodegenerative disorders in Nigerian Africans. Neurology 1984 34(3):361-362 
146. Stephany F, Mbaye PS, Jacquin-Cotton L, Ndiaye IP: Huntington chorea in Senegal. Dakar Med 1984, 29(1):75-83.

147. Samuels BL, Gelfand M: Huntington's chorea in a black Rhodesian family. S Afr Med J 1978, 54(16):648-651.

148. Glass J, Saffer DS: Huntington's chorea in a black family: a report of 2 cases. S Afr Med J 1979, 56(17):685-688,

149. Scrimgeour EM: Huntington's disease in Tanzania. J Med Genet 1981, 18(3):200-203.

150. Hayden MR, Beighton P: Genetic aspects of Huntington's chorea: results of a national survey. Am J Med Genet 1982, 11(2):135-141.

151. Scrimgeour EM: The Huntington's chorea register of Tanzania. East Afr Med J 1982, 59(4):280-282.

152. Scrimgeour EM, Pfumojena JW: Huntington disease in black Zimbabwean families living near the Mozambique border. Am J Med Genet 1992, 44(6):762-766.

153. Scrimgeour EM, Samman Y, Brock DJ: Huntington's disease in a Sudanese family from Khartoum. Human genetics 1995, 96(5):624-625.

154. Grunitzky EK, Gnamey DR, Nonon SA, Balogou A: Huntington disease in a large family in southern Togo. Ann Med Interne (Paris) 1995, 146(8):581-583.

155. Ferri $C P$, Prince $M$, Brayne C, Brodaty H, Fratiglioni L, Ganguli M, Hall K, Hasegawa K, Hendrie H, Huang Y, Jorm A, Mathers C, Menezes PR, Rimmer E, Scazufca M, Alzheimer's Disease International: Global prevalence of dementia: a Delphi consensus study. Lancet 2005, 366:2112-2117.

156. von Campenhausen BB S, Regina W, Kai B, Cristina S, Werner P, Wolfgang O, Uwe S, Karin Berger RD: Prevalence and incidence of Parkinson's disease in Europe. Eur Neuropsychopharmacol 2005, 15:473-490.

157. Ashford JW: Screening for mental disorders, dementia and Alzheimer's disease. Aging health 2008, 4(4):399-432.

158. Kim JH, Cheong HK, Lee CS, Yi SE, Park KW: The validity and reliability of a screening questionnaire for Parkinson's disease in a community. J Prev Med Public Health 2010, 43(1):9-17.

159. Sarangmath N, Rattihalli R, Ragothaman M, Gopalkrishna G, Doddaballapur S, Louis ED, Muthane UB: Validity of a modified Parkinson's disease screening questionnaire in India: effects of literacy of participants and medical training of screeners and implications for screening efforts in developing countries. Mov Disord 2005, 20(12):1550-1556.

160. Breteler MM, Bots ML, Ott A, Hofman A: Risk factors for vascular disease and dementia. Haemostasis 1998, 28(3-4):167-173.

161. Blennow K, de Leon MJ, Zetterberg H: Alzheimer's disease. Lancet 2006, 368(9533):387-403.

162. Elbaz A, Moisan F: Update in the epidemiology of Parkinson's disease. Curr Opin Neurol 2008, 21(4):454-460.

163. Brew BJ, Crowe SM, Landay A, Cysique LA, Guillemin G: Neurodegeneration and ageing in the HAART era. J Neuroimmune Pharmacol 2009, 4(2):163-174.

164. Population Division, DESA, United Nations, World Population Ageing 1950-2050. http://www.un.org/en/development/desa/population/ publications/pdf/ageing/WorldPopulationAgeingReport2013.pdf.

doi:10.1186/1471-2458-14-653

Cite this article as: Lekoubou et al: Epidemiology of neurodegenerative diseases in sub-Saharan Africa: a systematic review. BMC Public Health 2014 14:653.

\section{Submit your next manuscript to BioMed Central and take full advantage of:}

- Convenient online submission

- Thorough peer review

- No space constraints or color figure charges

- Immediate publication on acceptance

- Inclusion in PubMed, CAS, Scopus and Google Scholar

- Research which is freely available for redistribution 